TRANSACTIONS OF THE

AMERICAN MATHEMATICAL SOCIETY

Volume 365, Number 4, April 2013, Pages 1977-2030

S 0002-9947(2012)05676-5

Article electronically published on October 24, 2012

\title{
WRONSKIANS, CYCLIC GROUP ACTIONS, AND RIBBON TABLEAUX
}

\author{
KEVIN PURBHOO
}

\begin{abstract}
The Wronski map is a finite, $\mathrm{PGL}_{2}(\mathbb{C})$-equivariant morphism from the Grassmannian $\operatorname{Gr}(d, n)$ to a projective space (the projectivization of a vector space of polynomials). We consider the following problem. If $C_{r} \subset$ $\mathrm{PGL}_{2}(\mathbb{C})$ is a cyclic subgroup of order $r$, how may $C_{r}$-fixed points are in the fibre of the Wronski map over a $C_{r}$-fixed point in the base?

In this paper, we compute a general answer in terms of $r$-ribbon tableaux. When $r=2$, this computation gives the number of real points in the fibre of the Wronski map over a real polynomial with purely imaginary roots. More generally, we can compute the number of real points in certain intersections of Schubert varieties.

When $r$ divides $d(n-d)$ our main result says that the generic number of $C_{r}$-fixed points in the fibre is the number of standard $r$-ribbon tableaux of rectangular shape $(n-d)^{d}$. Computing by a different method, we show that the answer in this case is also given by the number of standard Young tableaux of shape $(n-d)^{d}$ that are invariant under $\frac{N}{r}$ iterations of jeu de taquin promotion. Together, these two results give a new proof of Rhoades' cyclic sieving theorem for promotion on rectangular tableaux.
\end{abstract}

We prove analogous results for dihedral group actions.

\section{INTRODUCTION}

1.1. Wronskians. The Wronski map is a finite morphism from a Grassmannian to a projective space of the same dimension, which is remarkably well behaved. In this paper, we consider the problem of counting points in a fibre of the Wronski map that are fixed by the action of a cyclic group. Our motivation comes from questions in real algebraic geometry and combinatorics, which shall be discussed later in the introduction. We begin by defining the Wronski map and recalling some of the properties that make it an interesting object of study in enumerative geometry.

For any non-negative integer $m$, let $\mathbb{C}_{m}[z]$ denote the vector space of polynomials of degree at most $m$. Fix integers $0<d<n$, and let $X:=\operatorname{Gr}\left(d, \mathbb{C}_{n-1}[z]\right)$ denote the Grassmannian of $d$-planes in the $n$-dimensional vector space $\mathbb{C}_{n-1}[z]$. The dimension of $X$ is $N:=d(n-d)$.

Received by the editors April 20, 2011 and, in revised form, July 26, 2011.

2010 Mathematics Subject Classification. Primary 14N10; Secondary 05E10, 14P05.

This research was partially supported by an NSERC discovery grant. 
If $x \in X$ is spanned by polynomials $f_{1}(z), \ldots, f_{d}(z) \in \mathbb{C}_{n-1}[z]$, we consider the Wronskian

$$
\operatorname{Wr}(x ; z):=\left|\begin{array}{ccc}
f_{1}(z) & \cdots & f_{d}(z) \\
f_{1}^{\prime}(z) & \cdots & f_{d}^{\prime}(z) \\
\vdots & \vdots & \vdots \\
f_{1}^{(d-1)}(z) & \cdots & f_{d}^{(d-1)}(z)
\end{array}\right| .
$$

It is easy to show that $\operatorname{Wr}(x ; z)$ is a non-zero polynomial of degree at most $N$, and different choices of basis for $x$ give the same Wronskian up to a scalar multiple. Thus $\operatorname{Wr}(x ; z)$ determines a well-defined element of projective space $\mathbb{P}\left(\mathbb{C}_{N}[z]\right)$. We adopt the convention that equations involving $\operatorname{Wr}(x ; z)$ hold only up to a scalar multiple, and should be properly interpreted in projective space. The morphism $\mathrm{Wr}: X \rightarrow \mathbb{P}\left(\mathbb{C}_{N}[z]\right), x \mapsto \mathrm{Wr}(x ; z)$ is called the Wronski map. There are many equivalent formulations. For example, the map $z \mapsto\left[f_{1}(z): \cdots: f_{d}(z)\right]$ defines a rational curve $\gamma: \mathbb{C P}^{1} \rightarrow \mathbb{C P}^{d-1}$; the roots $\mathrm{Wr}(x ; z)$ are the ramification points of $\gamma$. We refer the reader to the survey article [24] for a detailed background.

Eisenbud and Harris showed that the Wronski map is a finite morphism [5] of degree equal to the number of standard Young tableaux of rectangular shape $\square=(n-d)^{d}$. By studying the asymptotic behaviour of the fibres, Eremenko and Gabrielov refined this result 2]. They described a bijection between the set SYT $(\square)$ of all standard Young tableaux of shape $\square$, and points in the fibre $X(h(z)):=\mathrm{Wr}^{-1}(h(z))$ for certain polynomials $h(z) \in \mathbb{P}\left(\mathbb{C}_{N}[z]\right)$.

More generally, one can consider the restriction of the Wronski map to a Richardson variety. Let $\Lambda$ denote the set of all partitions $\lambda: \lambda^{1} \geq \lambda^{2} \geq \cdots \geq \lambda^{d} \geq 0$, with at most $d$ parts and $\lambda^{1} \leq n-d$, and consider the complete flags

$$
\begin{array}{ccc}
F_{\bullet}: F_{0} \subset F_{1} \subset \cdots \subset F_{n} & & \widetilde{F}_{\bullet}: \widetilde{F}_{0} \subset \widetilde{F}_{1} \subset \cdots \subset \widetilde{F}_{n} \\
F_{i}=z^{n-i} \mathbb{C}_{i-1}[z] & \text { and } & \widetilde{F}_{i}=\mathbb{C}_{i-1}[z] .
\end{array}
$$

For partitions $\lambda \supset \mu$ in $\Lambda$, the Richardson variety

$$
X_{\lambda / \mu}:=\left\{\begin{array}{l|l}
x \in X & \begin{array}{ll}
\operatorname{dim}\left(x \cap F_{n-d-\mu^{i}+i}\right) \geq i & \text { and } \\
\operatorname{dim}\left(x \cap \widetilde{F}_{\lambda^{d-i}+i}\right) \geq i, & \text { for } i=1, \ldots, d
\end{array}
\end{array}\right\}
$$

is the intersection of a Schubert variety (corresponding to $\mu$ ) and an opposite Schubert variety (corresponding to $\lambda$ ). We will say that $X_{\lambda / \mu}$ is compatible with a polynomial $h(z)$ if $|\lambda|=\operatorname{deg} h(z)$ and $|\mu|=\operatorname{mindeg} h(z):=\max \{m \geq 0 \mid$ $z^{m}$ divides $\left.h(z)\right\}$. This definition is motivated by the following fact.

Proposition 1.1 (See Section 4.1). Every point in $X(h(z))$ lies in a unique $h(z)$ compatible Richardson variety.

If we restrict the Wronski map to any Richardson variety $X_{\lambda / \mu}$, we obtain a finite morphism over the closure of the space of compatible polynomials. These restrictions interact nicely with the combinatorics of tableaux. The fibres are generically reduced, and the degree of this map is equal to the number of standard Young tableaux of skew shape $\lambda / \mu$. The correspondence of Eremenko and Gabrielov extends to this situation, giving a bijection for specially chosen polynomials.

One of the most remarkable facts about the Wronski map is the reality theorem of Mukhin, Tarasov and Varchenko (formerly the Shapiro-Shapiro conjecture). We 
say that a point $x \in X$ is real if the subspace of $\mathbb{C}_{n-1}[z]$ represented by $x$ has a basis $f_{1}(z), \ldots, f_{d}(z) \in \mathbb{R}_{n-1}[z]$.

Theorem 1.2 (Mukhin-Tarasov-Varchenko [18, 19]). Let $h(z) \in \mathbb{R}_{N}[z]$ be a polynomial of degree $N$ or $N-1$ whose roots are distinct real numbers. Then the fibre $X(h(z))$ is reduced and every point in the fibre is real.

Apart from being a very striking and surprising statement - one does not normally expect an algebraic system with real parameters to have only real solutions - this theorem has interesting combinatorial consequences. For example, it can be used to show that the correspondence of Eremenko and Gabrielov can be unambiguously extended to polynomials with real roots. This extension is not continuous, however it is discontinuous in an interesting way. In [20] we showed that the discontinuities encode the jeu de taquin theory on tableaux. Many of the results and methods from this previous work will be used in our present paper.

1.2. Cyclic group actions. The Mukhin-Tarasov-Varchenko theorem does not hold if $h(z) \in \mathbb{R}_{N}[z]$ is a real polynomial with complex roots. Computer experiments by Hauenstein, Hein, Martín del Campo and Sottile show that, in general, the number of real points in $X(h(z))$ depends in a complicated way on the polynomial $h(z)$, and not just on the number of real roots [10. However, there are other situations where the number of real solutions is predictable. The work in this paper was originally motivated by the following problem. Suppose $h(z) \in \mathbb{R}_{N}[z]$ is a real polynomial of degree at most $N$ with only pure imaginary roots. How many real points are in the fibre $X(h(z))$ ? Special cases of this problem were studied by Eremenko and Gabrielov, and used to construct instances of the pole placement problem in control theory with no real solutions [3].

To answer this (and other related questions), it is helpful to observe that the Wronski map is equivariant with respect to a $\mathrm{PGL}_{2}(\mathbb{C})$-action. The group $\mathrm{SL}_{2}(\mathbb{C})$ acts on each vector space $\mathbb{C}_{m}[z]$ by Möbius transformations: if $\phi=\left(\begin{array}{ll}\phi_{11} & \phi_{12} \\ \phi_{21} & \phi_{22}\end{array}\right) \in$ $\mathrm{SL}_{2}(\mathbb{C})$, we let

$$
\phi f(z):=\left(\phi_{21} z+\phi_{11}\right)^{m} f\left(\frac{\phi_{22} z+\phi_{12}}{\phi_{21} z+\phi_{11}}\right)
$$

for $f(z) \in \mathbb{C}_{m}[z]$. The action $\mathrm{SL}_{2}(\mathbb{C})$ on $\mathbb{C}_{n-1}[z]$ and $\mathbb{C}_{N}[z]$ induces an action of $\mathrm{PGL}_{2}(\mathbb{C})$ on $X$, and on $\mathbb{P}\left(\mathbb{C}_{N}[z]\right)$, and the Wronski map intertwines these two $\mathrm{PGL}_{2}(\mathbb{C})$-actions.

The involution $z \mapsto-z$ is given by $\phi=\left(\begin{array}{cc}-1 & 0 \\ 0 & 1\end{array}\right) \in \mathrm{PGL}_{2}(\mathbb{C})$, and (up to a sign) fixes every real polynomial $h(z)$ with only imaginary roots. Hence for these polynomials, $\phi$ gives an involution on the fibre $X(h(z))$. One can then show, using the Mukhin-Tarasov-Varchenko theorem, that the fixed points of this involution are exactly the real points in the fibre. We are left with the problem of counting the number of $\phi$-fixed points in the fibre over a $\phi$-fixed polynomial.

More generally, let $C_{r} \subset \mathrm{PGL}_{2}(\mathbb{C})$ be a cyclic subgroup of order $r$. We can consider the problem of counting $C_{r}$-fixed points in the fibre of a $C_{r}$-fixed polynomial in $\mathbb{P}\left(\mathbb{C}_{N}[z]\right)$. As any two of these cyclic subgroups are conjugate, we will generally take

$$
C_{r}:=\left\{\left(\begin{array}{cc}
e^{\pi i j / r} & 0 \\
0 & e^{-\pi i j / r}
\end{array}\right) \mid j=0, \ldots, r-1\right\} .
$$


Then, a polynomial $h(z)$ is $C_{r}$-fixed as an element of $\mathbb{P}\left(\mathbb{C}_{N}[z]\right)$ if and only if it is of the form

$$
h(z)=z^{m}\left(z^{r}+h_{1}\right)\left(z^{r}+h_{2}\right) \cdots\left(z^{r}+h_{\ell}\right),
$$

where $h_{1}, \ldots, h_{\ell} \in \mathbb{C}^{\times}, m \geq 0$ and $m+r \ell \leq N$. When we speak of a polynomial being $C_{r}$-fixed, we will always mean that it is fixed as an element of the appropriate projective space. Indeed, this is the only reasonable interpretation, since $C_{r}$ is a subgroup of $\mathrm{PGL}_{2}(\mathbb{C})$, not of $\mathrm{SL}_{2}(\mathbb{C})$.

Let $X^{r}$ denote the $C_{r}$-fixed subscheme of $X$. For any $h(z)$-compatible Richardson variety $X_{\lambda / \mu}$, we consider

$$
X_{\lambda / \mu}^{r}(h(z)):=X_{\lambda / \mu} \cap X^{r} \cap X(h(z)),
$$

the $C_{r}$-fixed points of the fibre of the Wronski map that lie in the Richardson variety $X_{\lambda / \mu}$.

Our main result is a combinatorial formula for the number of points in $X_{\lambda / \mu}^{r}(h(z))$ : we show that there is a bijection between these points and the set of standard $r$-ribbon tableaux of shape $\lambda / \mu$. When $r=1$, these are just standard Young tableaux, and the group is trivial; hence we recover the fact that $\left.\mathrm{Wr}\right|_{X_{\lambda / \mu}}$ has degree $|\operatorname{SYT}(\lambda / \mu)|$. The problem of counting real solutions when $h(z)$ is a real polynomial with pure imaginary roots corresponds to the special case of an involution, i.e. $r=2$. In the case where $r$ divides $N$, mindeg $h(z)=0$ and $\operatorname{deg} h(z)=N$, we can determine the answer in a second way - in terms of the jeu de taquin promotion operator on rectangular tableaux - giving a non-trivial combinatorial identity. These results can also be extended to dihedral group actions.

1.3. Ribbon tableaux. We now recall the definitions of the combinatorial objects that are relevant to the statements of our results. We assume some basic familiarity here, and refer the reader to [8, 25, 28, for a more thorough introduction.

Let $\lambda, \mu \in \Lambda$ be partitions with $\lambda \supset \mu$. By a tableau of shape $\lambda / \mu$, we will mean a filling of the boxes of the diagram of $\lambda / \mu$ with positive integer entries that are weakly increasing along rows and down columns. A tableau of shape $\lambda / \mu$ is a standard Young tableau if its entries are exactly the numbers $1, \ldots,|\lambda / \mu|$. We denote the set of all standard Young tableau of shape $\lambda / \mu$ by $\operatorname{SYT}(\lambda / \mu)$.

More generally, suppose $|\lambda / \mu|=r \ell$, and consider a tableau of shape $\lambda / \mu$ satisfying the following conditions:

(1) The entries are $1,2, \ldots, \ell$.

(2) The shape determined by the entries labelled $i$ is a (connected) $r$-ribbon; that is, a connected skew shape with $r$ boxes that does not contain a $2 \times 2$ square.

Such a tableau is called a standard $r$-ribbon tableau (also called a standard $r$-rim hook tableau). When $r=1$, this definition coincides with the definition of a standard Young tableaux. When $r=2$, the entries labelled $i$ must cover two adjacent boxes, and hence standard 2-ribbon tableaux are often called domino tableaux. An example for $r=5$ is given in Figure 1.1. We denote by $\operatorname{SRT}^{r}(\lambda / \mu)$ the set of all standard $r$-ribbon tableaux of shape $\lambda / \mu$.

Ribbon tableaux have been well studied. There are several formulae for $\left|\operatorname{SRT}^{r}(\lambda)\right|$, including hook-length formulae and character formulae (see [6] and the references therein). Lascoux, Leclerc and Thibon showed that evaluations of Kostka-Foulkes polynomials at roots of unity can be interpreted as enumerations of ribbon tableau. 


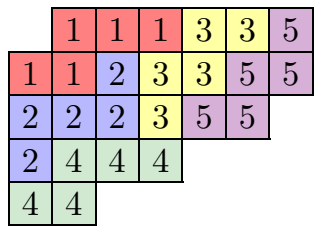

Figure 1.1. A standard 5-ribbon tableau of skew shape 77542/1.

In our case, the relevant Kostka-Foulkes polynomial is $K_{\lambda / \mu, 1^{k}}(q)($ where $k=|\lambda / \mu|)$, which is the generating function for $\operatorname{SYT}(\lambda / \mu)$ with respect to the charge statistic. The following is a special case of their result, which may be found in [15, Section $4]$.

Theorem 1.3 (Lascoux-Leclerc-Thibon). Suppose $|\lambda / \mu|=r \ell$. Then

$$
\left|\operatorname{SRT}^{r}(\lambda / \mu)\right|= \pm K_{\lambda / \mu, 1^{r \ell}}\left(e^{2 \pi i / r}\right) .
$$

In the case where $\lambda / \mu=\square$, the Kostka-Foulkes polynomial is up to a power of $q$ given by the $q$-analogue of the hook-length formula

$$
K_{\square, 1^{N}}(q)=q^{N(d-1) / 2} \frac{[N] !_{q}}{\prod_{\alpha \in \square}\left[h_{\alpha}\right]_{q}},
$$

and the sign appearing in Theorem 1.3 is $(-1)^{N(d-1) / 2}$.

These same numbers appear in another combinatorial context. For $\lambda \in \Lambda$, let $j: \operatorname{SYT}(\lambda) \rightarrow \operatorname{SYT}(\lambda)$ be the jeu de taquin promotion operator. For $T \in \operatorname{SYT}(\lambda)$, $\boldsymbol{j}(T)$ is defined by performing the following steps:

(1) Delete the entry 1 from $T$, leaving an empty box in the northwest corner.

(2) Slide the empty box through the remaining entries of $T$. (The empty box repeatedly swaps places with either its neighbour to the east or its neighbour to the south, whichever is smaller, until neither exists.)

(3) Decrement all entries by 1 and place a new entry $|\lambda|$ in the empty box. The result is $\boldsymbol{j}(T)$.

An example is given in Figure 1.2,

It is not hard to see that $j: \operatorname{SYT}(\lambda) \rightarrow \operatorname{SYT}(\lambda)$ is invertible, and hence generates a cyclic group action on $\operatorname{SYT}(\lambda)$. In the case where $\lambda=\square$, this operator has particularly nice properties. Haiman showed that $j^{N}(T)=T$ for all $T \in \operatorname{SYT}(\square)$ [9, hence we may think of this cyclic group as having order $N$. More generally, Rhoades proved the following cyclic sieving theorem for the promotion operator on SYT $(\square)$ [23] (see also [26, 30]).

Theorem 1.4 (Rhoades). Suppose $r$ divides $N$. Then the number of $\boldsymbol{j}^{N / r}$-fixed tableaux in SYT $(\square)$ is equal to $(-1)^{N(d-1) / r} K_{\square, 1^{N}}\left(e^{2 \pi i / r}\right)$.

When $r=1$, the evaluation of the Kostka-Foulkes polynomial $K_{\square, 1^{N}}(1)$ is just the ordinary Kostka number $K_{\square, 1^{N}}=|\mathrm{SYT}(\square)|$, and hence we recover Haiman's result. More generally this theorem gives a complete description of the orbit structure of promotion on SYT $(\square)$.

The proof of Theorem 1.4 is quite non-elementary. The idea is to show that the long cycle acts by promotion on the Kazhdan-Lusztig basis of the representation of 


$$
\begin{aligned}
T=\begin{array}{l|l|l}
\hline 1 & 2 & 5 \\
\hline 3 & 4 & 7 \\
\hline 6 & 8 & 9 \\
\hline
\end{array} \rightarrow \begin{array}{|l|l|l|}
\hline & 2 & 5 \\
\hline 3 & 4 & 7 \\
\hline 6 & 8 & 9 \\
\hline
\end{array} & \rightarrow \begin{array}{|l|l|l|}
\hline 2 & & 5 \\
\hline 3 & 4 & 7 \\
\hline 6 & 8 & 9 \\
\hline
\end{array} \\
& \rightarrow \begin{array}{|l|l|l|}
\hline 2 & 4 & 5 \\
\hline 3 & 7 & \\
\hline 6 & 8 & 9 \\
\hline
\end{array} \rightarrow \begin{array}{|l|l|l|l|}
\hline 2 & 4 & 5 \\
\hline 3 & & 7 \\
\hline 6 & 8 & 9 \\
\hline 2 & 4 & 5 \\
\hline 3 & 7 & 9 \\
\hline 6 & 8 & \\
\hline
\end{array} \rightarrow \begin{array}{|l|l|l|}
\hline 1 & 3 & 4 \\
\hline 2 & 6 & 8 \\
\hline 5 & 7 & 9 \\
\hline
\end{array}
\end{aligned}
$$

Figure 1.2. Promotion on a rectangular tableau $T \in \mathrm{SYT}(\square)$, with $n=6, d=3$.

the symmetric group $S_{N}$ associated to the partition $\square$; hence the number of fixed points is given as a character evaluation. In light of Theorem 1.3. Rhoades' result is equivalent to the following purely combinatorial theorem, for which there is no known bijective proof.

Theorem 1.5. Suppose r divides $N$. The number of $\boldsymbol{j}^{N / r}$-fixed tableaux in SYT $(\square)$ is equal to the number of standard r-ribbon tableaux of shape $\square$.

One consequence of our present work will be an alternate proof of Theorem 1.5. Our proof is direct in the sense that it does not require any knowledge of representation theory, Kostka-Foulkes polynomials, their evaluations at roots of unity, or any other algebraic formulae for the two quantities involved. On the other hand, the proof does not give a direct bijection between the two sets, nor does it suggest how to construct one (except in the $r=2$ case, where a bijection is known [16]). From our point of view, the two sets come from two different (and seemingly incompatible) ways of thinking about the problem of counting $C_{r}$-fixed points in the fibre of the Wronski map.

There is another operator on standard Young tableaux, closely related to promotion. For $\lambda \in \Lambda$, the evacuation operator $e: \operatorname{SYT}(\lambda) \rightarrow \operatorname{SYT}(\lambda)$ is

$$
\boldsymbol{e}:=\boldsymbol{j}_{1} \circ \boldsymbol{j}_{2} \circ \cdots \circ \boldsymbol{j}_{|\lambda|}
$$

where $\boldsymbol{j}_{i}$ is the promotion operator restricted to the entries $1, \ldots, i$. In other words, to compute $\boldsymbol{e}(T)$, for $T \in \mathrm{SYT}(\lambda)$, we first apply the promotion operator $\boldsymbol{j}=\boldsymbol{j}_{|\lambda|}$ to $T$. We then freeze the largest entry $|\lambda|$ in $\boldsymbol{j}(T)$, and apply promotion to the subtableau of $\boldsymbol{j}(T)$ consisting of entries $1, \ldots,|\lambda|-1$. At the next stage entries $|\lambda|$ and $|\lambda|-1$ are frozen, and we continue in this way until all entries are frozen. The result is $\boldsymbol{e}(T)$.

The operator $e$ is an involution on $\operatorname{SYT}(\lambda)$. In the case of a rectangular shape $\lambda=\square$, there is a very simple description of this involution: it coincides with the operation of rotating the tableau by $180^{\circ}$ and reversing the order of the entries (replacing each entry $k$ by $N+1-k$ ). From here, it is easy to see that $\boldsymbol{e} \circ \boldsymbol{j} \circ \boldsymbol{e}=\boldsymbol{j}^{-1}$, and hence $\boldsymbol{e}$ and $\boldsymbol{j}$ generate a dihedral group $D_{N}$ of order $2 N$ acting on SYT( $\left.\square\right)$. We may therefore consider the problem of counting tableaux in SYT $(\square)$ that are fixed by a dihedral subgroup $D_{r} \subset N$ of order $2 r$. If $N$ is odd, every dihedral subgroup of order $2 r$ is conjugate, so we may take $D_{r}$ to be generated by $\boldsymbol{e}$ and $j^{N / r}$. If $N$ is even, there are up to conjugacy two dihedral subgroups of order $2 r$ : one is generated by $\boldsymbol{e}$ and $\boldsymbol{j}^{N / r}$, the other by $\boldsymbol{e} \circ \boldsymbol{j}$ and $\boldsymbol{j}^{N / r}$. 


\begin{tabular}{|l|l|l|l|l|l|}
\hline 1 & 1 & 1 & 1 & 4 & 5 \\
\hline 1 & 2 & 3 & 4 & 4 & 5 \\
\hline 2 & 2 & 3 & 4 & 5 & 5 \\
\hline 2 & 3 & 3 & 4 & 5 & 6 \\
\hline 2 & 3 & 6 & 6 & 6 & 6 \\
\hline
\end{tabular}

\begin{tabular}{|l|l|l|l|l|l|}
\hline 1 & 1 & 1 & 1 & 3 & 3 \\
\hline 1 & 2 & 2 & 3 & 3 & 4 \\
\hline 2 & 2 & 3 & 3 & 4 & 4 \\
\hline 2 & 3 & 3 & 4 & 4 & 5 \\
\hline 3 & 3 & 5 & 5 & 5 & 5 \\
\hline
\end{tabular}

Figure 1.3. Examples of tableaux in $\operatorname{RRT}^{5}(\square)$ and $\widehat{\operatorname{RRT}}^{5}(\square)$, with $n=11, d=5$.

To answer this question in a manner analogous to Theorem 1.5 we consider two special types of ribbon tableaux. For each $\lambda \in \Lambda$, let $\lambda^{\vee} \in \Lambda$ denote the dual partition: $\left(\lambda^{\vee}\right)^{i}:=n-d-\lambda^{d+1-i}$ for $i=1, \ldots, d$. We will say a tableau is rotationally-invariant if it is fixed by the operation of rotating $180^{\circ}$ inside the rectangle $\square$, and replacing each entry $k$ by $m+1-k$, where $m$ is the largest entry. For a tableau to be rotationally-invariant, its shape must be of the form $\lambda / \lambda^{\vee}$, where $\lambda \supset \lambda^{\vee}$.

Suppose $|\lambda / \mu|=r \ell$. We define $\operatorname{RRT}^{r}(\lambda / \mu)$ to be the set of all rotationallyinvariant standard $r$-ribbon tableaux (this is empty if $\mu \neq \lambda^{\vee}$ ). If $\ell$ is even, we also define $\widehat{\operatorname{RRT}}^{r}(\lambda / \mu)$ to be the set of rotationally-invariant tableaux with entries $1,2, \ldots, \ell-1$, in which the entries not equal to $\frac{\ell}{2}$ form $r$-ribbons, and the entries equal to $\frac{\ell}{2}$ form a $2 r$-ribbon. Some examples are given in Figure 1.3 . We note that $\widehat{\operatorname{RRT}}^{r}(\lambda / \mu)$ is always empty if $d$ and $n$ are both even.

Theorem 1.6. Suppose $r$ divides $N$.

(i) The number of tableaux in SYT( $\square$ ) fixed by both $\boldsymbol{e}$ and $\boldsymbol{j}^{N / r}$ is equal to $\left|\operatorname{RRT}^{r}(\square)\right|$.

(ii) The number of tableaux in SYT( $\square$ ) fixed by both $\boldsymbol{e} \circ \boldsymbol{j}$ and $\boldsymbol{j}^{N / r}$ is equal to $\left|\operatorname{RRT}^{r}(\square)\right|$ if $\frac{N}{r}$ is odd, and $\left|\widehat{\operatorname{RRT}}^{r}(\square)\right|$ if $\frac{N}{r}$ is even.

In the $r=1$ case we are computing the number of standard Young tableaux of rectangular shape fixed by $\boldsymbol{e}$, or $\boldsymbol{e} \circ \boldsymbol{j}$, which were previously studied in [23, 29]. Since these are involutions, the quantities involved are related to the $r=2$ case of a cyclic group action. The general case of Theorem [1.6] appears to be new, and follows from dihedral group analogues of our main theorems.

1.4. Statements of results. Our main result relates the problem of counting $C_{r^{-}}$ fixed points in the fibre of the Wronski map to enumeration of $r$-ribbon tableaux.

Theorem 1.7. Let $h(z)$ be a generic $C_{r}$-fixed polynomial of the form (1.2). For any $h(z)$-compatible Richardson variety $X_{\lambda / \mu}, X_{\lambda / \mu}^{r}(h(z))$ is a reduced finite scheme. The number of points in $X_{\lambda / \mu}^{r}(h(z))$ is equal to the number of standard r-ribbon tableaux of shape $\lambda / \mu$.

When $N=r \ell$ and

$$
h(z)=\left(z^{r}+h_{1}\right)\left(z^{r}+h_{2}\right) \cdots\left(z^{r}+h_{\ell}\right),
$$

where $h_{1}, \ldots, h_{\ell} \in \mathbb{C}^{\times}$, there is only one compatible Richardson variety, $X=X_{\square}$ itself. In this case, we write $X^{r}(h(z))$ for the $C_{r}$-fixed points in the fibre $X(h(z))$. Theorem 1.7 then states that $X^{r}(h(z))$ is generically reduced, of size equal to $\left|\mathrm{SRT}^{r}(\square)\right|$. In this case, we will also show the following. 
Theorem 1.8. Let $h(z)$ be a generic polynomial of the form (1.3). The number of points in $X^{r}(h(z))$ is equal to the number of $j^{N / r}$-fixed tableaux in SYT( $\left.\square\right)$.

Putting Theorems 1.7 and 1.8 together, we deduce Theorem 1.5 .

These two results are proved by considering the fibres over certain special classes of polynomials. To prove Theorem 1.7, we change our field from the complex numbers to a field of Puiseux series $\mathbb{F}\{\{u\}\}$, where $\mathbb{F}$ is an algebraically closed field containing $\mathbb{C}$. Since both fields are algebraically closed of characteristic zero, this does not change the number of points. However, it allows us to make some nonalgebraic assumptions about the roots: specifically, we can consider polynomials $C_{r}$-fixed polynomials $h(z)$, where $h_{1}, \ldots, h_{\ell}$ have distinct valuations. In this case, we establish a bijection between $\operatorname{SRT}^{r}(\lambda / \mu)$ and $X_{\lambda / \mu}^{r}(h(z))$. These methods are related to the asymptotic approach used by Eremenko and Gabrielov in 2, and build on the theory developed in 20 .

To prove Theorem 1.8, we consider the case where all $N$ roots of $h(z)$ lie on the unit circle. As the unit circle is $\mathrm{PGL}_{2}(\mathbb{C})$-equivalent to the real line, we can apply methods based on the Mukhin-Tarasov-Varchenko theorem. The proof is essentially a straightforward interpretation of results proved in 20. From the point of view of this argument, it is clear why the tableau need to be of rectangular shape in Theorem 1.4 points corresponding to non-rectangular shaped tableaux lie in some proper Richardson variety of $X$; the image under the Wronski map is a compatible polynomial, which cannot have $N$ roots on the unit circle.

The case of an involution is rather special. There are a number of interpretations of the $C_{2}$-fixed points in the fibre. For example, if we assume that $h(z)$ is a real polynomial with only pure imaginary roots, then the $C_{2}$-fixed points are precisely the real points in the fibre, and Theorem 1.7 states that the number of these in any compatible Richardson variety $X_{\lambda / \mu}$ is equal to the number of domino tableaux of shape $\lambda / \mu$. Alternatively we may assume that the roots of $h(z)$ are all real, and thereby deduce Theorem 1.7 directly from results in 20 . We can also describe what happens when the roots of $h(z)$ are not necessarily distinct.

Another consequence in real algebraic geometry concerns maximally inflected real rational curves in projective space. Consider a real rational curve $\gamma: \mathbb{R P}^{1} \rightarrow$ $\mathbb{R P}^{d-1}, z \mapsto\left[f_{1}(z): \cdots: f_{d}(z)\right]$, where $f_{1}(z), \ldots, f_{d}(z) \in \mathbb{R}_{n-1}[z]$ are linearly independent. We say $\gamma$ is maximally inflected if it has only simple, real ramification points. Equivalently for the point $x \in X$ spanned by $f_{1}(z), \ldots, f_{d}(z), \operatorname{Wr}(x ; z)$ has $N$ or $N-1$ distinct roots, all of which are real. Consider the space $\mathcal{R}$ of all maximally inflected real rational curves $\gamma: \mathbb{R} \mathbb{P}^{1} \rightarrow \mathbb{R} \mathbb{P}^{d-1}$ of the above form. The results used to prove Theorem 1.8 imply that the connected components of $\mathcal{R}$ are in a one-to-one or two-to-one correspondence with the $j$-orbits on SYT $(\square)$.

Theorem 1.9. Let $S$ be the group of order two if $d$ is even, or the trivial group if $d$ is odd, and let $s \in S$ be the element of order $|S|$.

(i) The connected components of $\mathcal{R}$ are smooth, and are in bijection with the set $\operatorname{Orb}(\boldsymbol{j}, s)$ of orbits of $(\boldsymbol{j}, s)$ acting on $\mathrm{SYT}(\square) \times S$.

(ii) If $\theta \in \mathrm{PGL}_{d}(\mathbb{R})$ is a reflection, then the automorphism $\gamma \mapsto \theta \circ \gamma$ of $\mathcal{R}$ descends to the action of (id, $s)$ on $\operatorname{Orb}(\boldsymbol{j}, s)$.

(iii) If $\psi \in \mathrm{PGL}_{2}(\mathbb{R})$ is a reflection, then the automorphism $\gamma \mapsto \gamma \circ \psi$ of $\mathcal{R}$ descends to the action of $\left(\boldsymbol{e}, s^{\lfloor d / 2\rfloor}\right)$ on $\operatorname{Orb}(\boldsymbol{j}, s)$. 
Both Theorems 1.7 and 1.8 have extensions to actions of a dihedral group. Let $D_{r} \subset \mathrm{PGL}_{2}(\mathbb{C})$ be the dihedral subgroup of order $2 r$ generated by $C_{r}$ and the reflection $\left(\begin{array}{ll}0 & 1 \\ 1 & 0\end{array}\right)$. A polynomial $h(z)$ is $D_{r}$-fixed if it is of the form (1.2) where $2 m+r \ell=N$, and $\left\{h_{1}, \ldots, h_{\ell}\right\}$ is invariant under $z \mapsto \frac{1}{z}$. There are three distinct generic types:

(1) $\ell$ is odd, one of the $h_{i}$ is equal to \pm 1 , and the rest form generic inverse pairs;

(2) $\ell$ is even, all of the $h_{i}$ form generic inverse pairs (none are equal to \pm 1 );

(3) $\ell$ is even, one of the $h_{i}$ is equal to 1 , another is equal to -1 , and the rest form generic inverse pairs.

Theorem 1.10. Let $h(z)$ be a $D_{r}$-fixed polynomial of one of the three generic types above, and let $X_{\lambda / \mu}$ be a compatible Richardson variety. Then $X_{\lambda / \mu}^{r}(h(z))$ is a reduced finite scheme. Moreover:

(i) If $h(z)$ is of type (1) or (2), the number of $D_{r}$-fixed points in $X_{\lambda / \mu}(h(z))$ is equal to $\left|\operatorname{RRT}^{r}(\lambda / \mu)\right|$.

(ii) If $h(z)$ is of type (3), the number of $D_{r}$-fixed points in $X_{\lambda / \mu}(h(z))$ is equal

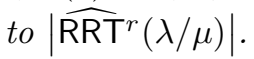

In the case where $m=0$ (hence $h(z)$ is of the form (1.3) and $\lambda / \mu=\square$ ), we also prove:

Theorem 1.11. Let $h(z)$ be a $D_{r}$-fixed polynomial of one of the three generic types, with $m=0$.

(i) If $h(z)$ is of type (1) or (2), the number of $D_{r}$-fixed points in $X(h(z))$ is equal to the number tableaux in SYT( $\square)$ fixed by both $\boldsymbol{e}$ and $\boldsymbol{j}^{N / r}$.

(ii) If $h(z)$ is of type (1) or (3), the number of $D_{r}$-fixed points in $X(h(z))$ is equal to the number tableaux in SYT( $\square)$ fixed by both $\boldsymbol{e} \circ \boldsymbol{j}$ and $\boldsymbol{j}^{N / r}$.

Putting Theorems 1.10 and 1.11 together, we deduce Theorem 1.6

The main idea behind the proof of Theorem 1.10 is to show that the action of $\left(\begin{array}{ll}0 & 1 \\ 1 & 0\end{array}\right)$ on the fibre models the action of a $180^{\circ}$ rotation on the tableaux. This follows fairly readily from the results we use to study $C_{r}$-fixed points. In the type (3) case, a small amount of additional analysis is needed, because we can't choose the $h_{i}$ to have distinct valuations. Theorem 1.11 uses essentially the same ideas as Theorem 1.8 .

Remark 1.12. It is worth mentioning that virtually every result in this paper should have an analogue for the Orthogonal Grassmannian OG $(n, 2 n+1)$ that could be proved similarly using the results of [20, 21]. In particular, Theorems 1.5 and 1.6 should have analogues where tableaux are replaced by shifted tableaux of staircase shape. We have chosen not to check all of the details here. An open question is whether it is possible to use our approach to prove analogues of Theorems 1.5 and 1.6 for semistandard tableaux, such as [23. Theorems 1.4 and 1.5]. The geometry of this question is closely related to the discussion in Section [3.2, where fibre of the Wronski map is replaced by a Schubert intersection. A more ambitious problem is to generalize Theorem 1.7 to counting $C_{r}$-fixed points of these Schubert intersections.

1.5. Outline. This paper is effectively divided into two parts, which can be read independently of each other. The first part comprises Sections 2 and 3 . In these 
two sections, we will use arguments that apply in the case where $h(z)$ has $N$ roots that lie on a circle in $\mathbb{C P}^{1}$. For such $h(z)$, one can define a correspondence between $\mathrm{SYT}(\square)$ and points in $X(h(z))$. We review the important properties of this correspondence in Section 2, and use it to prove Theorems 1.8, 1.9 and 1.11, In Section 3 , we consider the special case of counting points in $X(h(z))$ that are fixed by an involution in $\mathrm{PGL}_{2}(\mathbb{C})$. We study this in a variety of ways, which include giving different interpretations of the problem, and giving alternate proofs of some of our main results. More generally we explain how to count involution-fixed points in certain intersections of Schubert varieties.

Sections 4, 5 and 6 form the second part of the paper. The focus in these three sections is on understanding the restriction of the Wronski map to the fixed point scheme $X^{r}$, explicitly in terms of the Plücker coordinates. In Section 4 , we describe the components of $X^{r}$, their coordinate rings in terms of Plücker coordinates, and their initial ideals with respect to certain weight functions. In Section 5 we describe a very general way to associate a tableau to any point in $X$, working over a valuation ring. Using this association, we prove Theorem 1.7. In Section 6, we extend our results to dihedral group actions and prove Theorem 1.10.

\section{Roots on A CIRCLE}

2.1. Lifting paths and jeu de taquin. In the case where the roots of $h(z)$ all lie on a circle in $\mathbb{C P}^{1}$, points in the fibre of the Wronski map $X(h(z))$ can be identified with standard Young tableaux of shape $\square$. Moreover, any element of $\mathrm{PGL}_{2}(\mathbb{C})$ that fixes $h(z)$ can be identified with an operator on SYT $(\square)$. We begin with a review of these results, wherein our circle is just $\mathbb{R P}^{1} \subset \mathbb{C P}^{1}$. The proofs may be found in [20], but our presentation here more closely follows [22].

Let $\mathbf{a}=\left\{a_{1}, \ldots, a_{N}\right\}$ be a multiset, where $a_{1}, \ldots, a_{N} \in \mathbb{R} \mathbb{P}^{1}$. Following the notation of [20], we will think of a polynomial in terms of its roots, and write $X(\mathbf{a}):=X(h(z))$, where

$$
h(z)=\prod_{a_{i} \neq \infty}\left(z+a_{i}\right) .
$$

Suppose $\mathbf{a}$ is a set (i.e. $a_{i} \neq a_{j}$ for $i \neq j$ ), and let $x \in X(\mathbf{a})$. We wish to associate to $x$ a tableau $T_{x} \in \mathrm{SYT}(\square)$. To accomplish this, we consider the total order $\preceq$ on $\mathbb{R P}^{1}$, defined by $a \preceq b$, if either $a=b,|a|<|b|$, or $0<a=-b<\infty$. Since a is a set, we may assume without loss of generality that

$$
a_{1} \prec a_{2} \prec \cdots \prec a_{N} .
$$

Now, for $k=0, \ldots, N$ and $t \in[0,1]$, let

$$
\mathbf{a}_{k, t}=\left\{t a_{1}, t a_{2}, \ldots, t a_{k}, a_{k+1}, a_{k+2}, \ldots, a_{N}\right\} .
$$

For all $t \in(0,1], \mathbf{a}_{k, t}$ is a set, and hence by Theorem 1.2, the fibres $X\left(\mathbf{a}_{k, t}\right)$ are reduced. It follows that there is a unique (continuous) lifting of the path $\mathbf{a}_{k, t}, t \in[0,1]$, to a path $x_{k, t} \in X\left(\mathbf{a}_{k, t}\right)$, where $x_{k, 1}=x$. Now, since $0 \in \mathbf{a}_{k, 0}$ with multiplicity $k$, and $x_{k, 0} \in X\left(\mathbf{a}_{k, 0}\right)$, we have mindeg $\operatorname{Wr}\left(x_{k, 0} ; z\right)=k$. By Proposition 1.1 $x_{k, 0}$ lies in a unique Richardson variety $X_{\nu / \lambda_{k}}$, where $\lambda_{k} \vdash k$.

Theorem 2.1. The sequence $\lambda_{0}, \lambda_{1}, \ldots, \lambda_{N}$ is a chain of partitions

$$
\varnothing=\lambda_{0} \subsetneq \lambda_{1} \subsetneq \cdots \subsetneq \lambda_{N}=\square .
$$

Moreover, no two points of $X(\mathbf{a})$ give rise to the same chain. 
We define $T_{x} \in \mathrm{SYT}(\square)$ to be the tableau that encodes the chain of partitions $\lambda_{0} \subsetneq \lambda_{1} \subsetneq \cdots \subsetneq \lambda_{N} ; T_{x}$ has entry $k$ in the box corresponding to $\lambda_{k} / \lambda_{k-1}$.

Informally, we are defining $T_{x}$ by moving the point $x$ so that $k$ smallest roots of $\mathrm{Wr}(x ; z)$ are sent to zero, and seeing where $x$ ends up. Alternatively we can obtain $T_{x}$ by sending the $N-k$ largest roots to $\infty$. For $k=0, \ldots, N$ and $t \in[0,1]$, let

$$
\mathbf{a}_{k, t}^{\prime}=\left\{a_{1}, a_{2}, \ldots, a_{k}, t^{-1} a_{k+1}, t^{-1} a_{k+2}, \ldots, t^{-1} a_{N}\right\},
$$

and lift to $x_{k, t}^{\prime} \in X\left(\mathbf{a}_{k, t}^{\prime}\right)$, where $x_{k, 1}^{\prime}=x$. In this case, since $\infty \in \mathbf{a}_{k, 0}$ with multiplicity $N-k$, we have $\operatorname{deg} \operatorname{Wr}\left(x_{k, 0}^{\prime} ; z\right)=k$, and hence $x_{k, 0}^{\prime}$ lies in a unique Richardson variety $X_{\lambda_{k}^{\prime} / \mu}$, where $\lambda_{k}^{\prime} \vdash k$. It turns out that $\lambda_{k}^{\prime}$ is equal to the partition $\lambda_{k}$ constructed above, and hence both constructions yield the same tableau. (This can be seen from a third construction in which one considers

$$
\mathbf{a}_{k, t}^{\prime \prime}=\left\{t a_{1}, t a_{2}, \ldots, t a_{k}, t^{-1} a_{k+1}, t^{-1} a_{k+2}, \ldots, t^{-1} a_{N}\right\} .
$$

The lifted path $x_{k, t}^{\prime \prime} \in X\left(\mathbf{a}_{k, t}^{\prime \prime}\right)$ (with $x_{k, 1}^{\prime \prime}=x$ ) is compatible with the previous two, in that we must have $x_{k, 0}^{\prime \prime} \in X_{\lambda_{k}^{\prime} / \lambda_{k}}$. Hence this Richardson variety is non-empty, which implies $\lambda_{k}^{\prime}=\lambda_{k}$. See [20, Section 6] for details.)

This definition of $T_{x}$ was based on the idea of lifting a path of subsets of $\mathbb{R} \mathbb{P}^{1}$ to a path in $X$. More generally, if $\left(a_{1}\right)_{t}, \ldots,\left(a_{N}\right)_{t}, t \in[0,1]$, are paths in $\mathbb{R P}^{1}$ and $\mathbf{a}_{t}=\left\{\left(a_{1}\right)_{t}, \ldots,\left(a_{N}\right)_{t}\right\}$ has the property that $X\left(\mathbf{a}_{t}\right)$ is reduced for all $t$, we may consider lifting $\mathbf{a}_{t}$ to a path $x_{t} \in X\left(\mathbf{a}_{t}\right)$. We can understand such liftings in terms of the associated tableaux.

In the case where $\left(a_{1}\right)_{t}, \ldots,\left(a_{N}\right)_{t}$ remain in the same $\preceq$-order, the tableau associated to $x_{t}$ remains constant.

Theorem 2.2. Suppose $\left(a_{1}\right)_{t} \prec\left(a_{2}\right)_{t} \prec \cdots \prec\left(a_{N}\right)_{t}$ for all $t \in[0,1]$. If $x_{t} \in X\left(\mathbf{a}_{t}\right)$ is a lifting of $\mathbf{a}_{t}$ to $X$, then $T_{x_{0}}=T_{x_{1}}$.

The relationship between $T_{x_{0}}$ and $T_{x_{1}}$ is more interesting if $\left(a_{1}\right)_{t}, \ldots,\left(a_{N}\right)_{t}$ do not remain in the same $\preceq$-order. First note that by Theorem 1.2 , the relation between $x_{0}$ and $x_{1}$ (and hence between $T_{x_{0}}$ and $T_{x_{1}}$ ) depends only on the homotopy class of the path $\mathbf{a}_{t}$. It suffices, therefore, to describe this relationship in the simplest possible case:

$$
\begin{gathered}
\left(a_{1}\right)_{t} \prec \cdots \prec\left(a_{k}\right)_{t},\left(a_{k+1}\right)_{t} \prec \cdots \prec\left(a_{N}\right)_{t} \quad \text { for all } t \in[0,1], \\
\left(a_{k}\right)_{0} \prec\left(a_{k+1}\right)_{0} \quad \text { and } \quad\left(a_{k+1}\right)_{1} \prec\left(a_{k}\right)_{1} .
\end{gathered}
$$

Any path is homotopic to a concatenation of paths of the form (2.3), so the answer in this simple case gives a complete description of the relationship between $T_{x_{0}}$ and $T_{x_{1}}$ in general.

Theorem 2.3. Suppose that $\mathbf{a}_{t}$ is a path of the form (2.3), and $x_{t} \in X\left(\mathbf{a}_{t}\right)$ is a lifting to $X$.

(i) If $\left(a_{k}\right)_{0}$ and $\left(a_{k+1}\right)_{0}$ have the same sign, then $T_{x_{0}}=T_{x_{1}}$.

(ii) If $k$ and $k+1$ are in the same row or column of $T_{x_{0}}$, then $T_{x_{0}}=T_{x_{1}}$.

(iii) If $\left(a_{k}\right)_{0}$ and $\left(a_{k+1}\right)_{0}$ have opposite signs, and $k$ and $k+1$ are in different rows and columns of $T_{x_{0}}$, then $T_{x_{1}}$ is obtained from $T_{x_{0}}$ by switching the positions of $k$ and $k+1$.

We will mainly be interested in the case where $\left(a_{k}\right)_{0}$ and $\left(a_{k+1}\right)_{0}$ have opposite signs. To see what is going on here, it is helpful to think of replacing the entries $1, \ldots, N$ of the tableau associated to a point $x \in X(\mathbf{a})$ with real entries from the 
set a. Specifically, we will replace $k$ by $a_{k}$, where $a_{1} \prec a_{2} \prec \cdots \prec a_{N}$. We refer to the resulting object as a real valued tableau, and write $T_{x}(\mathbf{a})$ for the real valued tableau associated to $x$.

In case (ii), the tableau $T_{x}$ does not change. Hence in the real valued tableau $T_{x_{t}}\left(\mathbf{a}_{t}\right)$ the relative $\preceq$-order of entries does not change. But since the relative order of $\left(a_{k}\right)_{t}$ and $\left(a_{k+1}\right)_{t}$ does change, these must switch places in $T_{x_{t}}\left(\mathbf{a}_{t}\right)$. In case (iii), the positions of $k$ and $k+1$ are changing along with the relative order of $\left(a_{k}\right)_{t}$ and $\left(a_{k+1}\right)_{t}$. In the real valued tableau, these two changes nullify each other, and the entries $\left(a_{k}\right)_{t}$ and $\left(a_{k+1}\right)_{t}$ remain where they are in $T_{x_{t}}\left(\mathbf{a}_{t}\right)$. In both cases, this is a very small instance of a Schützenberger slide. The box containing entry $\left(a_{k}\right)_{t}$ is sliding through the subtableau consisting of the single box $\left(a_{k+1}\right)_{t}$. As the next example illustrates, the big picture emerges by considering what happens when a negative number passes by several positive numbers.

Example 2.4. Consider a path $\mathbf{a}_{t}=\left\{\left(a_{1}\right)_{t}, 3,8,12,19,21,27,34,39\right\}$, where $\left(a_{1}\right)_{0}=$ $0,\left(a_{1}\right)_{1}=\infty$, and the path $\left(a_{1}\right)_{t}$ traverses the negative real numbers. Let $x_{t} \in$ $X\left(\mathbf{a}_{t}\right)$ be a lifting such that $x_{0}$ is associated to the real valued tableau

$$
T_{x_{0}}\left(\mathbf{a}_{0}\right)=\begin{array}{|c|c|c|}
\hline 0 & 3 & 19 \\
\hline 8 & 12 & 27 \\
\hline 21 & 34 & 39 \\
\hline
\end{array}
$$

As $\left(a_{1}\right)_{t}$ passes each of the other entries, the two entries switch places if and only if they are in the same row or column.

\begin{tabular}{|c|c|c|}
\hline 0 & 3 & 19 \\
\hline 8 & 12 & 27 \\
\hline 21 & 34 & 39 \\
\hline
\end{tabular}$\rightarrow$\begin{tabular}{|c|c|c|}
\hline 3 & -5 & 19 \\
\hline 8 & 12 & 27 \\
\hline 21 & 34 & 39 \\
\hline
\end{tabular}$\rightarrow$\begin{tabular}{|c|c|c|c|}
3 & -10 & 19 \\
\hline 8 & 12 & 27 \\
\hline 21 & 34 & 39 \\
\hline
\end{tabular}

$\rightarrow$\begin{tabular}{|c|c|c|}
\hline 3 & 12 & 19 \\
\hline 8 & -15 & 27 \\
\hline 21 & 34 & 39 \\
\hline
\end{tabular}$\rightarrow$\begin{tabular}{|c|c|c|}
\hline 3 & 12 & 19 \\
\hline 8 & -20 & 27 \\
\hline 21 & 34 & 39 \\
\hline
\end{tabular}$\rightarrow$\begin{tabular}{|c|c|c|}
\hline 3 & 12 & 19 \\
\hline 8 & -25 & 27 \\
\hline 21 & 34 & 39 \\
\hline
\end{tabular}

$\rightarrow$\begin{tabular}{|c|c|c|}
\hline 3 & 12 & 19 \\
\hline 8 & 27 & -30 \\
\hline 21 & 34 & 39 \\
\hline
\end{tabular}$\rightarrow$\begin{tabular}{|c|c|c|}
\hline 3 & 12 & 19 \\
\hline 8 & 27 & -35 \\
\hline 21 & 34 & 39 \\
\hline
\end{tabular}$\rightarrow$\begin{tabular}{|c|c|c|}
\hline 3 & 12 & 19 \\
\hline 8 & 27 & 39 \\
\hline 21 & 34 & $\infty$ \\
\hline
\end{tabular}

Comparing the steps here with the example in Figure 1.2, we see that $T_{x_{1}}=\boldsymbol{j}\left(T_{x_{0}}\right)$, where $\boldsymbol{j}$ is the promotion operator on the tableaux.

In general, we can model both the promotion and evacuation operators on SYT $(\square)$ in terms of lifting a suitable path $\mathbf{a}_{t}$ that begins at a set of positive 
real numbers:

$$
0<\left(a_{1}\right)_{0}<\left(a_{2}\right)_{0}<\cdots<\left(a_{N}\right)_{0}<\infty .
$$

We will describe promotion as the lifting of a loop $\mathbf{a}_{t}, t \in[0,1]$. Let $\left(a_{1}\right)_{t}$ be a path that first decreases from $\left(a_{1}\right)_{0}$ to 0 , then moves along the negative real axis to $\infty$, and finally decreases from $\infty$ to the value $\left(a_{1}\right)_{1}=\left(a_{N}\right)_{0}$. Meanwhile, for $k=2, \ldots, N$, let $\left(a_{k}\right)_{t}$ move monotonically from $\left(a_{k}\right)_{0}$ to $\left(a_{k}\right)_{1}=\left(a_{k-1}\right)_{0}$. Thus $\mathbf{a}_{t}$ is a loop that cyclically rotates the elements $\left(a_{1}\right)_{0}, \ldots,\left(a_{N}\right)_{0}$. (See Figure 2.1]) In terms of the $\preceq$-order, $\left(a_{1}\right)_{t}$ is changing places with each of $\left(a_{k}\right)_{t}, k \geq 2$, and doing so with opposite signs. Thus, in the real valued tableau associated to a lifting, the box containing $\left(a_{1}\right)_{t}$ begins in the northwest corner and slides through all the other entries, ending up in the southeast corner. Hence we see that for any lifting $x_{t} \in X\left(\mathbf{a}_{t}\right)$ of such a loop, we have $T_{x_{1}}=\boldsymbol{j}\left(T_{x_{0}}\right)$.

Evacuation, on the other hand, is described by lifting a path $\mathbf{a}_{t}, t \in[0,1]$, for which $\mathbf{a}_{1}=-\mathbf{a}_{0}$. For $k=1, \ldots, N$, let $\left(a_{k}\right)_{t}$ be a path that begins at $\left(a_{k}\right)_{0}$, decreases to 0 , and continues moving monotonically until it reaches $\left(a_{k}\right)_{1}=$ $-\left(a_{N+1-k}\right)_{0}$. (See Figure 2.2.) This describes a unique homotopy class of paths. To show this homotopy class models evacuation, it is convenient to consider a specific representative, which we will describe by dividing up the interval $[0,1]$ into $N$ subintervals $\left[\frac{i-1}{N}, \frac{i}{N}\right], i=1, \ldots, N$. On the first interval $\left[0, \frac{1}{N}\right],\left(a_{1}\right)_{t}$ moves monotonically to $\left(a_{1}\right)_{1 / N}=-\left(a_{N}\right)_{0}$. Meanwhile, for $k=2, \ldots, N,\left(a_{k}\right)_{t}$ moves monotonically to $\left(a_{k}\right)_{1 / N}=\left(a_{k-1}\right)_{0}$. Hence $\left(a_{1}\right)_{t}$ has changed places in the $\preceq$ order with each of the other $\left(a_{k}\right)_{t}$, with opposite signs; this models the promotion operator $\boldsymbol{j}=\boldsymbol{j}_{N}$. On the next interval $\left[\frac{1}{N}, \frac{2}{N}\right],\left(a_{1}\right)_{t}=-\left(a_{N}\right)_{0}$ remains constant, while $\left(a_{2}\right)_{t}$ moves monotonically to $-\left(a_{N-1}\right)_{0}$, and for $k=3, \ldots, N,\left(a_{k}\right)_{t}$ moves monotonically to $\left(a_{k-2}\right)_{0}$. Hence $\left(a_{2}\right)_{t}$ is changing places in the $\preceq$-order, with each $\left(a_{k}\right)_{t}, k \geq 3$, but does not pass $\left(a_{1}\right)_{t}$. This models the promotion operator restricted to the $N-1$ smallest entries of the tableau, which is $\boldsymbol{j}_{N-1}$. In general, on the $i^{\text {th }}$ interval $\left[\frac{i-1}{N}, \frac{i}{N}\right],\left(a_{1}\right)_{t}, \ldots,\left(a_{i-1}\right)_{t}$ have reached their final positions, and $\left(a_{i}\right)_{t}$ moves to $-\left(a_{N+1-i}\right)_{t}$, while $\left(a_{k}\right)_{t}$ moves to $\left(a_{k-i}\right)_{0}$; this models $\boldsymbol{j}_{i}$. Hence the complete path models $\boldsymbol{e}=\boldsymbol{j}_{1} \circ \boldsymbol{j}_{2} \circ \cdots \circ \boldsymbol{j}_{N}$; that is, for any lifting $x_{t} \in X\left(\mathbf{a}_{t}\right)$, we have $T_{x_{1}}=\boldsymbol{e}\left(T_{x_{0}}\right)$.

From this perspective it is clear why $\boldsymbol{e}$ is an involution: if we reverse the roles of the positive and negative real numbers, we see that the reverse of a path that models $e$ also models $e$, hence $e=e^{-1}$.

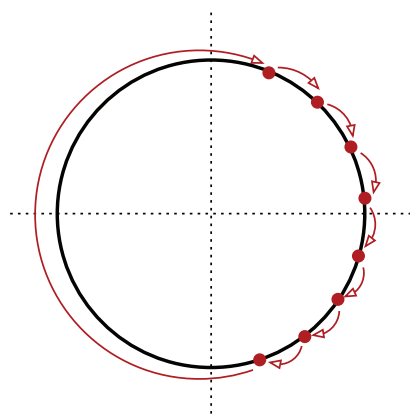

Figure 2.1. A loop that models promotion. The circle is $\mathbb{R P}^{1}$, drawn with 0 south, $\infty$ north, 1 east, and -1 west. 

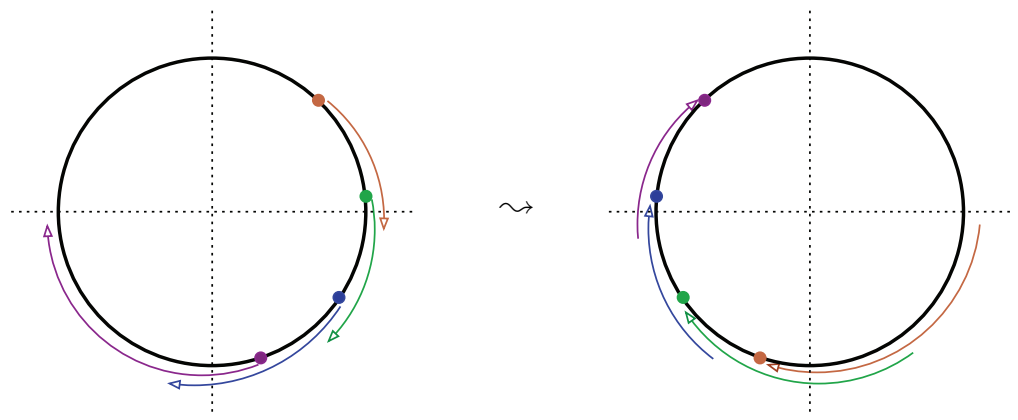

Figure 2.2. A path that models evacuation.

2.2. The $\mathrm{PGL}_{2}(\mathbb{R})$-action on real valued tableaux. The group $\mathrm{PGL}_{2}(\mathbb{C})$ acts on $\mathbb{C P}^{1}$ by Möbius transformations. If $a \in \mathbb{C P}^{1}$ and $\phi=\left(\begin{array}{ll}\phi_{11} & \phi_{12} \\ \phi_{21} & \phi_{22}\end{array}\right)$, then

$$
\phi(a):=\frac{\phi_{11} a+\phi_{12}}{\phi_{21} a+\phi_{22}} .
$$

This action restricts to an action of $\mathrm{PGL}_{2}(\mathbb{R})$ on $\mathbb{R P}^{1}$.

Let $\mathbf{a}=\left\{a_{1}, \ldots, a_{N}\right\}$ be a subset of $\mathbb{R P}^{1}$, let $x \in X(\mathbf{a})$ and let $\phi \in \mathrm{PGL}_{2}(\mathbb{R})$. Since the Wronski map is $\mathrm{PGL}_{2}(\mathbb{C})$-equivariant, we have $\phi(x) \in X(\phi(\mathbf{a}))$, where $\phi(\mathbf{a})=\left\{\phi\left(a_{1}\right), \ldots, \phi\left(a_{N}\right)\right\}$ is also a subset of $\mathbb{R P}^{1}$. This defines an action on real valued tableaux. If $T=T_{x}(\mathbf{a})$ is the real valued tableau associated to $x$, we define

$$
\phi(T):=T_{\phi(x)}(\phi(\mathbf{a})) .
$$

If $\phi$ is in the connected component of $\left(\begin{array}{ll}1 & 0 \\ 0 & 1\end{array}\right)$, we can compute this action explicitly, using Theorem 2.3. Let $\phi_{t}, t \in[0,1]$, be any path in $\mathrm{PGL}_{2}(\mathbb{R})$ from $\phi_{0}=\left(\begin{array}{ll}1 & 0 \\ 0 & 1\end{array}\right)$ to $\phi_{1}=\phi$. Then $x_{t}=\phi_{t}(x)$ is a lifting of the path $\phi_{t}(\mathbf{a})$. Theorem 2.3 therefore tells us exactly how the tableau $T_{\phi_{1}(x)}\left(\phi_{1}(\mathbf{a})\right)=\phi(T)$ is related to $T_{\phi_{0}(x)}\left(\phi_{0}(\mathbf{a})\right)=T$.

If $\phi$ is not in the connected component of the identity, then we can write $\phi=$ $\left(\begin{array}{cc}-1 & 0 \\ 0 & 1\end{array}\right) \phi^{\prime}$, or $\phi=\left(\begin{array}{ll}0 & 1 \\ 1 & 0\end{array}\right) \phi^{\prime \prime}$, where $\phi^{\prime}, \phi^{\prime \prime}$ are in the connected component of the identity matrix. The next lemma tells us how to compute the actions of $\left(\begin{array}{cc}-1 & 0 \\ 0 & 1\end{array}\right)$ and $\left(\begin{array}{ll}0 & 1 \\ 1 & 0\end{array}\right)$.

Lemma 2.5. Suppose that

$$
\left|a_{1}\right|<\left|a_{2}\right|<\cdots<\left|a_{N}\right|,
$$

and let $T$ be a real valued tableau with entries from the set $\mathbf{a}$.

(i) $\left(\begin{array}{rr}-1 & 0 \\ 0 & 1\end{array}\right) T=-T$, where $-T$ is obtained from $T$ by replacing $a_{k}$ with $-a_{k}$, for $k=1, \ldots, N$.

(ii) $\left(\begin{array}{ll}0 & 1 \\ 1 & 0\end{array}\right) T=T^{\vee}$, where $T^{\vee}$ is obtained from $T$ by rotating $180^{\circ}$ and replacing $a_{k}$ with $\frac{1}{a_{k}}$, for $k=1, \ldots, N$.

If we have a set $\mathbf{a}$ for which $a_{i}=-a_{j}$ for some $i \neq j$, then Lemma 2.5 cannot be used directly. The problem is that if we replace (2.4) by the weaker condition $a_{1} \prec a_{2} \prec \cdots \prec a_{N}$, then $-T$ and $T^{\vee}$ may not be real valued tableaux. The operation $a \mapsto-a$ is not $\preceq$-order preserving, and $a \mapsto \frac{1}{a}$ is not $\preceq$-order reversing. In this case, we can still compute $\left(\begin{array}{cc}-1 & 0 \\ 0 & 1\end{array}\right) T$ and $\left(\begin{array}{ll}0 & 1 \\ 1 & 0\end{array}\right) T$, but we need to do so by 
perturbing the point a, which introduces additional sliding. We consider examples of this in Section 3 .

Proof. Let $x \in X(\mathbf{a})$ and $\hat{x}=\left(\begin{array}{rr}-1 & 0 \\ 0 & 1\end{array}\right) x$. Let $\hat{\mathbf{a}}=\left(\begin{array}{rr}-1 & 0 \\ 0 & 1\end{array}\right) \mathbf{a}=\left\{-a_{1}, \ldots,-a_{N}\right\}$. Recall from Section 2.1 that the tableaux $T_{x}$ is computed by lifting a path $\mathbf{a}_{k, t}$ (see (2.1)) to a path $x_{k, t}$. Similarly the tableau $T_{\hat{x}}=\left(\begin{array}{rr}-1 & 0 \\ 0 & 1\end{array}\right) T_{x}$ is computed by lifting a path $\hat{\mathbf{a}}_{k, t}$ to a path $\hat{x}_{k, t}$. Now, since $a_{1} \prec a_{2} \prec \cdots \prec a_{N}$ and $-a_{1} \prec-a_{2} \prec \cdots \prec$ $-a_{N}, \hat{\mathbf{a}}_{k, t}=\left(\begin{array}{cc}-1 & 0 \\ 0 & 1\end{array}\right) \mathbf{a}_{k, t}$ for all $k=0, \ldots, N, t \in[0,1]$, and therefore $\hat{x}_{k, t}=x_{k, t}$. Finally, since $\left(\begin{array}{cc}-1 & 0 \\ 0 & 1\end{array}\right)$ fixes every Richardson variety, $x_{k, 0} \in X_{\nu, \lambda_{k}}$ if and only if $\hat{x}_{k, 0} \in X_{\nu, \lambda_{k}}$, and we see that $T_{x}=T_{\hat{x}}$, which is equivalent to (i).

For (ii), the argument is similar. This time, let $\hat{x}=\left(\begin{array}{ll}0 & 1 \\ 1 & 0\end{array}\right) x$ and $\hat{\mathbf{a}}=\left(\begin{array}{ll}0 & 1 \\ 1 & 0\end{array}\right) \mathbf{a}=$ $\left\{\frac{1}{a_{1}}, \ldots, \frac{1}{a_{N}}\right\}$. Since $\frac{1}{a_{N}} \prec \frac{1}{a_{N-1}} \prec \cdots \prec \frac{1}{a_{1}}$, the path $\hat{\mathbf{a}}_{N-k, t}$ is equal to $\left(\begin{array}{cc}0 & 1 \\ 1 & 0\end{array}\right) \mathbf{a}_{k, t}^{\prime}$, where $\mathbf{a}_{k, t}^{\prime}$ is the path (2.1) that appears in the alternate definition of $T_{x}$. Thus we obtain $\hat{x}_{N-k, t}=\left(\begin{array}{ll}0 & 1 \\ 1 & 0\end{array}\right) x_{k, t}^{\prime}$, and deduce the result from the fact that $\left(\begin{array}{ll}0 & 1 \\ 1 & 0\end{array}\right) X_{\nu / \lambda_{k}}=$ $X_{\lambda_{k}^{\vee} / \nu^{\vee}}$.

Now suppose that $\phi(\mathbf{a})=\mathbf{a}$ for some set $\mathbf{a}$. In this case, the real valued tableau $\phi\left(T_{x}(\mathbf{a})\right)$ has the same entries as $T_{x}(\mathbf{a})$. By identifying real valued tableaux with entries from the set a with SYT $(\square)$, we can regard $\phi$ as an operator on SYT $(\square)$. We note that this identification is always defined with respect to a particular choice of $\phi$-fixed set $\mathbf{a}$, and that changing this set will change the identification.

We now show that if $a_{1}, \ldots, a_{N}$ are positive real numbers, then the operator defined by $\phi$ belongs to the dihedral subgroup $D_{2 N}$ generated by the promotion and evacuation operators $\boldsymbol{j}, \boldsymbol{e}: \mathrm{SYT}(\square) \rightarrow \mathrm{SYT}(\square)$. Moreover, for a suitable choice of a, we will realize $\boldsymbol{j}$ and $\boldsymbol{e}$ by elements of $\mathrm{PGL}_{2}(\mathbb{R})$. From here it will be a simple matter to prove Theorems 1.8 and 1.11 .

Lemma 2.6. Let $\phi \in \mathrm{PGL}_{2}(\mathbb{R})$, and suppose that $\phi(\mathbf{a})=\mathbf{a}$, where $0<a_{1}<$ $\cdots<a_{N}<\infty$. If $\phi$ is in the connected component of $\left(\begin{array}{ll}1 & 0 \\ 0 & 1\end{array}\right)$, then the action of $\phi$ on SYT $(\square)$ coincides with $j^{k}$ for some integer $k$. If $\phi$ is not in the connected component of $\left(\begin{array}{ll}1 & 0 \\ 0 & 1\end{array}\right)$, then the action of $\phi$ on $\mathrm{SYT}(\square)$ coincides with $\boldsymbol{e} \circ \boldsymbol{j}^{k}$, for some integer $k$.

Proof. If $\phi$ is in the connected component of the identity, then $\phi(T)$ is defined by lifting a path $\phi_{t}(\mathbf{a}), t \in[0,1]$. Such a path must cyclically rotate the elements of a by some amount; i.e. there is some $k$ such that $\phi\left(a_{i}\right)=a_{i+k(\bmod N)}$. Then $\phi_{t}(\mathbf{a})$ is homotopic to the path that models $\boldsymbol{j}^{k}$, and hence $\phi(T)=\boldsymbol{j}^{k}(T)$.

Now suppose $\phi$ is not in the connected component of the identity. Write $\phi=$ $\left(\begin{array}{cc}-1 & 0 \\ 0 & 1\end{array}\right) \phi^{\prime}$, and consider the action of $\phi^{\prime}$ on real valued tableau. The path $\phi_{t}^{\prime}(\mathbf{a})$ used to define this action preserves cyclic order, and $\phi^{\prime}(\mathbf{a})=\left(\begin{array}{cc}-1 & 0 \\ 0 & 1\end{array}\right) \mathbf{a}=\left\{-a_{1}, \ldots,-a_{N}\right\}$. Hence $\phi_{t}^{\prime}(\mathbf{a})$ must be homotopic to a path that cyclically rotates the elements of $\mathbf{a}$ by some amount, followed by a path that models evacuation. In other words, if $T_{x}$ is the tableau corresponding to a point $x \in X(\mathbf{a})$, then the tableau corresponding to $\phi^{\prime}(x)$ is $e \circ j^{k}\left(T_{x}\right)$, for some $k$. By Lemma 2.5(i), this is also the tableau corresponding to $\phi(x)$.

In order to explicitly realize $\boldsymbol{j}$ and $\boldsymbol{e}$ by elements of $\mathrm{PGL}_{2}(\mathbb{R})$ and relate these to the statements of Theorems 1.8 and 1.11, it will be convenient to work with the unit circle $S^{1} \subset \mathbb{C P}^{1}$ rather than $\mathbb{R P}^{1}$. To relate the two, we will use a Möbius 
transformation that maps "most" of the unit circle to the positive real numbers. Let $\eta:=e^{i \varepsilon}$ where $0<\varepsilon<\frac{\pi}{N}$, and

$$
\psi:=\left(\begin{array}{ll}
1+\bar{\eta} & -(1+\eta) \\
1+\eta & -(1+\bar{\eta})
\end{array}\right)
$$

Then $\psi$ defines a Möbius transformation which sends $S^{1}$ to $\mathbb{R P}^{1}$, with $\psi(\eta)=$ $0, \psi(\bar{\eta})=\infty$ and $\psi(-1)=1$. We let $\mathrm{PGL}_{2}\left(S^{1}\right):=\psi \mathrm{PGL}_{2}(\mathbb{R}) \psi^{-1}$ denote the subgroup of $\mathrm{PGL}_{2}(\mathbb{C})$ that fixes the unit circle.

For $k=1, \ldots, N$, let $a_{k}=e^{(2 k-1) \pi i / N}$. Since $a_{1}, \ldots, a_{N}$ lie on the long arc of the unit circle between $\eta$ and $\bar{\eta}$, we have

$$
0<\psi\left(a_{1}\right)<\psi\left(a_{2}\right)<\cdots<\psi\left(a_{N}\right)<\infty .
$$

For each point $x \in X(\mathbf{a})$, we define the tableau $T_{x} \in \mathrm{SYT}(\square)$ associated to $x$ to be $T_{\psi(x)}$. Using this identification, any $\phi \in \mathrm{PGL}_{2}\left(S^{1}\right)$ that fixes a defines an operator on SYT $(\square)$.

Lemma 2.7. With $a_{1}, \ldots, a_{N}$ as above, the $\mathrm{PGL}_{2}\left(S^{1}\right)$ group elements

$$
\left(\begin{array}{cc}
e^{-\pi i / N} & 0 \\
0 & e^{\pi i / N}
\end{array}\right) \quad \text { and } \quad\left(\begin{array}{ll}
0 & 1 \\
1 & 0
\end{array}\right)
$$

fix $\mathbf{a}$, and act on $\mathrm{SYT}(\square)$ as $\boldsymbol{j}$ and $\boldsymbol{e}$, respectively.

Together with Lemma 2.5(ii), Lemma 2.7 shows that for $T \in \mathrm{SYT}(\square), \boldsymbol{e}(T)$ is the tableau obtained by rotating $T$ by $180^{\circ}$ and replacing each entry $k$ by $N+1-k$. This is well known, but not entirely obvious.

Proof. We argue as in the proof of Lemma 2.6. That both of these Möbius transformations fix a is easy to check. In particular, $\left(\begin{array}{cc}e^{-\pi i / N} & 0 \\ 0 & e^{\pi i / N}\end{array}\right) a_{i}=a_{i+1(\bmod N)}$, which implies that $\phi$ acts as $\boldsymbol{j}^{1}$. For the action of $\left(\begin{array}{ll}0 & 1 \\ 1 & 0\end{array}\right)$, note that $\left(\begin{array}{ll}0 & 1 \\ 1 & 0\end{array}\right)$ commutes with $\psi$, so it is enough to show that $\left(\begin{array}{ll}0 & 1 \\ 1 & 0\end{array}\right)$ acts as $\boldsymbol{e}$ on real valued tableaux with entries $\psi(\mathbf{a})$. But now, $\left(\begin{array}{ll}0 & 1 \\ 1 & 0\end{array}\right)=\left(\begin{array}{cc}-1 & 0 \\ 0 & 1\end{array}\right)\left(\begin{array}{cc}0 & -1 \\ 1 & 0\end{array}\right)$, and the action of $\left(\begin{array}{cc}0 & -1 \\ 1 & 0\end{array}\right)$ on $\psi(\mathbf{a})$ is computed by

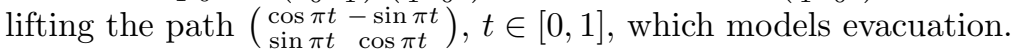

We are now in a position to prove Theorems 1.8 and 1.11

Proof of Theorem 1.8. Let $h(z)=\left(z+a_{1}\right)\left(z+a_{2}\right) \cdots\left(z+a_{N}\right)=z^{N}+(-1)^{N}$. Then $h(z)$ is a $C_{r}$-fixed polynomial, and the fibre $X(h(z))=X(\mathbf{a})$ is reduced. Therefore the number of $C_{r}$-fixed points in $X(\mathbf{a})$ is the generic answer. A point $x \in X(\mathbf{a})$ is $C_{r}$-fixed if and only if the corresponding tableau $T_{x}$ is $C_{r}$-fixed. However, by Lemma 2.7, the generator of $C_{r}$ acts as $j^{N / r}$ on SYT( $\left.\square\right)$. Hence the $C_{r}$-fixed points in $X(\mathbf{a})$ are in bijection with $j^{N / r}$-fixed tableaux in SYT $(\square)$.

Proof of Theorem 1.11, Case (i) is similar to the proof of Theorem 1.8. The polynomial $h(z)=\left(z+a_{1}\right)\left(z+a_{2}\right) \cdots\left(z+a_{N}\right)=z^{N}+(-1)^{N}$ is fixed by $C_{r}$ and also by $\left(\begin{array}{ll}0 & 1 \\ 1 & 0\end{array}\right)$. Moreover, $h(-1) \neq 0$, so $h(z)$ is of type (1) or (2), depending on whether $\frac{N}{r}$ is odd or even. By Lemma 2.7, the generators of $D_{r}$ act as $j^{N / r}$ and $e$, and hence the $D_{r}$-fixed points of $X(\mathbf{a})$ are in bijection with tableaux in SYT $(\square)$ fixed by $j^{N / r}$ and $e$. 
For case (ii), we need to consider a different polynomial. Let $h(z)=z^{N}-(-1)^{N}$. Since $h(-1)=0$, this is of type (1) or (3), depending on whether $\frac{N}{r}$ is odd or even. Let

$$
\phi=\left(\begin{array}{cc}
e^{-\pi i / 2 N} & 0 \\
0 & e^{\pi i / 2 N}
\end{array}\right) .
$$

Then $h(z)=\left(z+\phi\left(a_{1}\right)\right)\left(z+\phi\left(a_{2}\right)\right) \cdots\left(z+\phi\left(a_{N}\right)\right)$, so $X(h(z))=\phi(X(\mathbf{a}))$. A point $x \in X(h(z))$ is fixed by $D_{r}$ if and only if $\phi^{-1}(x) \in X(\mathbf{a})$ is fixed by $\phi^{-1} D_{r} \phi$, i.e. by

and

$$
\phi^{-1}\left(\begin{array}{cc}
e^{-\pi i / r} & 0 \\
0 & e^{\pi i / r}
\end{array}\right) \phi=\left(\begin{array}{cc}
e^{-\pi i / r} & 0 \\
0 & e^{\pi i / r}
\end{array}\right)
$$

$$
\phi^{-1}\left(\begin{array}{ll}
0 & 1 \\
1 & 0
\end{array}\right) \phi=\left(\begin{array}{ll}
0 & 1 \\
1 & 0
\end{array}\right)\left(\begin{array}{cc}
e^{-\pi i / N} & 0 \\
0 & e^{\pi i / N}
\end{array}\right) .
$$

By Lemma 2.7, the tableaux corresponding to points $\phi^{-1}(x)$ fixed by these two group elements are those fixed by $\boldsymbol{j}^{N / r}$ and $\boldsymbol{e} \circ \boldsymbol{j}$.

2.3. Maximally inflected real rational curves. As an application of the results from this section, we will briefly discuss the classification of maximally inflected real rational curves. Let $\gamma: \mathbb{R} \mathbb{P}^{1} \rightarrow \mathbb{R P}^{d-1}$ be a real parameterized curve

$$
\gamma: z \mapsto\left[f_{1}(z): \cdots: f_{d}(z)\right]
$$

with $f_{1}(z), \ldots, f_{d}(z) \in \mathbb{R}_{n-1}[z]$ linearly independent. A ramification point of $\gamma$ is a point $z \in \mathbb{R P}^{1}$ at which $\gamma(z), \gamma^{\prime}(z), \gamma^{\prime \prime}(z), \ldots, \gamma^{(d-1)}(z)$ do not span $\mathbb{R}^{d}$. When $d=2$, a ramification point is a critical point of the rational function $\frac{f_{1}(z)}{f_{2}(z)}$. When $d=3, \gamma$ is a planar curve, and the simplest example of a real ramification point of $\gamma$ is an inflection point. In general, if $\langle\gamma\rangle$ denotes the vector space spanned by $f_{1}(z), \ldots, f_{d}(z)$, it is not hard to see that a ramification point of $\gamma$ is a root of $\operatorname{Wr}(\langle\gamma\rangle ; z)$; hence $\gamma$ can have at most $N$ real ramification points. The space $\mathcal{R}$ of maximally inflected real rational curves is the algebraic set of all curves $\gamma$ of this form with exactly $N$ distinct, real ramification points. These curves and their relationship to Theorem 1.2 were first studied in 13 .

One interpretation of Theorem 1.9 is that the different "types" of curves in $\mathcal{R}$ correspond to the $D_{N}$-orbits on SYT $(\square)$, where $D_{N}$ is the dihedral group of order $2 N$ generated by $\boldsymbol{j}$ and $\boldsymbol{e}$. Here, two curves are of the same type if one is obtained from the other by deforming continuously within $\mathcal{R}$, and possibly composing with a reflection of $\mathbb{R P}^{1}$ or $\mathbb{R P}^{d-1}$ (or both). The actual statement of Theorem 1.9 is about a finer invariant than this notion of type, in which one does not identify curves related by reflections. On the other hand, curves of different types are fundamentally different because they degenerate in different ways when the ramification points collide (see [13, 24]); hence the type of a curve is the coarsest invariant one wants for any classification.

The analysis of the components of $\mathcal{R}$ is slightly different depending on whether $d$ is odd or even. This is because $\mathbb{R P}^{d-1}$ is orientable if $d$ is even, and non-orientable if $d$ is odd. When $d$ is even, we put $S:=\{ \pm 1\}$, and $s:=-1$. Let $\operatorname{Wr}(\gamma ; z)$ denote the right hand side of (1.1). Although the coordinates of $\gamma(z)$ are only defined up to a real scalar multiple, since $d$ is even, the $\operatorname{sign} \operatorname{Wr}(\gamma ; z)$ is unchanged by any real rescaling of $\left[f_{1}(z): \cdots: f_{d}(z)\right]$. We can therefore define the sign of $\gamma$ to be

$$
\epsilon_{\gamma}:=\lim _{z \rightarrow 0^{-}} \operatorname{sign}(\operatorname{Wr}(\gamma, z)) .
$$


If $\operatorname{Wr}(\gamma ; 0) \neq 0$, then $\epsilon_{\gamma}$ is just the $\operatorname{sign}$ of $\operatorname{Wr}(\gamma ; 0)$. When $d$ is odd there is no well-defined notion of the sign of a curve: we put $S:=\{1\}, s:=1$, and $\epsilon_{\gamma}:=1$ for every $\gamma \in \mathcal{R}$.

The group $\mathrm{PGL}_{2}(\mathbb{R})$ acts on $\mathcal{R}$ : if $\phi \in \mathrm{PGL}_{2}(\mathbb{R})$, then $\phi(\gamma)(z):=\left[\phi\left(f_{1}\right)(z)\right.$ : $\left.\cdots: \phi\left(f_{d}\right)(z)\right]$ is well defined. Let $\mathrm{PGL}_{2}(\mathbb{R})^{\circ} \subset \mathrm{PGL}_{2}(\mathbb{R})$ denote the connected component of the identity element. It is clear that if $\phi \in \mathrm{PGL}_{2}(\mathbb{R})^{\circ}$, then $\gamma$ and $\phi(\gamma)$ are in the same connected component of $\mathcal{R}$. We also have an action of $\mathrm{PGL}_{d}(\mathbb{R})$ on $\mathcal{R}$, given by $\gamma \mapsto \theta \circ \gamma$ for $\theta \in \mathrm{PGL}_{d}(\mathbb{R})$. If $\theta$ is in the connected component of the identity element, then $\gamma$ and $\theta \circ \gamma$ are in the same component of $\mathcal{R}$. Otherwise, we have $\langle\gamma\rangle=\langle\theta \circ \gamma\rangle$ and $\epsilon_{\gamma}=s \epsilon_{\theta \circ \gamma}$.

We now associate to each $\gamma \in \mathcal{R}$ a discrete invariant $\mathcal{O}_{\gamma}$, which will be a subset of SYT $(\square) \times S$. Let $x=\langle\gamma\rangle$, and

$$
M_{x}:=\left\{\phi \in \mathrm{PGL}_{2}(\mathbb{R})^{\circ} \mid \phi(x) \in X(\mathbf{a}) \text { for } \mathbf{a} \subset \mathbb{R}_{>0}\right\} .
$$

We define $\mathcal{O}_{\gamma}$ to be the set of pairs

$$
\mathcal{O}_{\gamma}:=\left\{\left(T_{\phi(x)}, \epsilon_{\phi(\gamma)}\right) \mid \phi \in M_{x}\right\} .
$$

Our next goal is to show that $\mathcal{O}_{\gamma}$ has the properties needed to prove Theorem 1.9.

Lemma 2.8. For every curve $\gamma \in \mathcal{R}, \mathcal{O}_{\gamma} \in \operatorname{Orb}(\boldsymbol{j}, s)$; i.e. $\mathcal{O}_{\gamma}$ is an orbit of $(\boldsymbol{j}, s)$ acting on $\mathrm{SYT}(\square) \times S$.

Proof. Let $x=\langle\gamma\rangle$, and $\phi \in M_{x}$. Then $\phi(x) \in X(\mathbf{a})$, where $\mathbf{a}$ is a set of the form $\mathbf{a}=\left\{0<a_{1}<\cdots<a_{N}<0\right\}$. We must show that $\mathcal{O}_{\gamma}$ is equal to the $(\boldsymbol{j}, s)$-orbit of $\left(T_{\phi(x)}, \epsilon_{\phi(\gamma)}\right)$.

For any other $\phi^{\prime} \in M_{x}$, we can write $\phi^{\prime}=\psi \phi$, where $\psi \in \mathrm{PGL}_{2}(\mathbb{R})^{\circ}$. Since $\phi^{\prime}(x) \in X(\psi(\mathbf{a}))$ we must have

$$
0<\psi\left(a_{k}\right)<\psi\left(a_{k+1}\right)<\cdots<\psi\left(a_{N}\right)<\psi\left(a_{1}\right)<\psi\left(a_{k-1}\right)<\infty
$$

for some integer $k$. As in the proof of Lemma 2.6, this implies that $T_{\phi^{\prime}(x)}=$ $T_{\psi(\phi(x))}=\boldsymbol{j}^{k}\left(T_{\phi(x)}\right)$. If $d$ is even, the sign of $\operatorname{Wr}(\gamma ; z)$ changes each time we pass a root; hence we also have $\epsilon_{\phi^{\prime}(\gamma)}=s^{k} \epsilon_{\phi(\gamma)}$. Thus $\mathcal{O}_{\gamma}$ is contained in the $(\boldsymbol{j}, s)$-orbit of $\left(T_{\phi(x)}, \epsilon_{\phi(\gamma)}\right)$.

For the reverse inclusion, choose any $\psi \in \mathrm{PGL}_{2}(\mathbb{R})^{\circ}$ such that

$$
0<\psi\left(a_{2}\right)<\psi\left(a_{3}\right)<\cdots<\psi\left(a_{N}\right)<\psi\left(a_{1}\right)<\infty .
$$

If $\phi^{\prime}=\psi \phi$, then $\phi^{\prime} \in M_{x}$ and $\left(T_{\phi^{\prime}(x)}, \epsilon_{\phi^{\prime}(\gamma)}\right)=\left(j\left(T_{\phi(x)}\right), s \epsilon_{\phi(\gamma)}\right)$. Thus $\mathcal{O}_{\gamma}$ contains the $(\boldsymbol{j}, s)$-orbit of $\left(T_{\phi(x)}, \epsilon_{\phi(\gamma)}\right)$.

Lemma 2.9. For every orbit $\mathcal{O} \in \operatorname{Orb}(\boldsymbol{j}, s)$, there exists a curve $\gamma$ such that $\mathcal{O}_{\gamma}=$ $\mathcal{O}$.

Proof. Choose any $(T, \epsilon) \in \mathcal{O}$, any $\mathbf{a}=\left\{0<a_{1}<\cdots<a_{N}<\infty\right\}$, any $x \in X(\mathbf{a})$ such that $T_{x}=T$, and any $\gamma$ such that $\langle\gamma\rangle=x$. If $\epsilon_{\gamma} \neq \epsilon$, then replace $\gamma$ by $\theta \circ \gamma$ for any reflection $\theta \in \mathrm{PGL}_{d}(\mathbb{R})$. Then $(T, \epsilon) \in \mathcal{O}_{\gamma}$, and thus by Lemma 2.8, $\mathcal{O}_{\gamma}=\mathcal{O}$.

Lemma 2.10. Two curves $\gamma$ and $\gamma^{\prime}$ are in the same connected component of $\mathcal{R}$ if and only if $\mathcal{O}_{\gamma}=\mathcal{O}_{\gamma^{\prime}}$. 
Proof. Suppose $\gamma, \gamma^{\prime}$ are in the same connected component of $\mathcal{R}$. Let $\gamma_{t}, t \in[0,1]$, be a path in $\mathcal{R}$ from $\gamma_{0}=\gamma$ to $\gamma_{1}=\gamma^{\prime}$. Let $x_{t}=\left\langle\gamma_{t}\right\rangle$. There exists a continuous path $\phi_{t}$ in $\mathrm{PGL}_{2}(\mathbb{R})^{\circ}$ such that $\phi_{t} \in M_{x_{t}}$. By Theorem 2.2, $T_{\phi_{t}\left(x_{t}\right)}$ is constant for all $t \in[0,1]$. Since $\operatorname{Wr}\left(\gamma_{t} ; 0\right) \neq 0$ for all $t \in[0,1], \epsilon_{\phi_{t}\left(\gamma_{t}\right)}$ is also constant. Thus we have $\left(T_{\phi_{0}\left(x_{0}\right)}, \epsilon_{\phi_{0}\left(\gamma_{0}\right)}\right)=\left(T_{\phi_{1}\left(x_{1}\right)}, \epsilon_{\phi_{1}\left(\gamma_{1}\right)}\right) \in \mathcal{O}_{\gamma} \cap \mathcal{O}_{\gamma^{\prime}}$, which implies $\mathcal{O}_{\gamma}=\mathcal{O}_{\gamma^{\prime}}$.

Conversely, suppose that $\mathcal{O}_{\gamma}=\mathcal{O}_{\gamma^{\prime}}$. Let $x=\langle\gamma\rangle, x^{\prime}=\left\langle\gamma^{\prime}\right\rangle$. Choose any $(T, \epsilon) \in$ $\mathcal{O}_{\gamma}$, and any $\phi \in M_{x}, \phi^{\prime} \in M_{x^{\prime}}$, so that $(T, \epsilon)=\left(T_{\phi(x)}, \epsilon_{\phi(\gamma)}\right)=\left(T_{\phi^{\prime}\left(x^{\prime}\right)}, \epsilon_{\phi^{\prime}\left(\gamma^{\prime}\right)}\right)$. Choose a path of sets $\mathbf{a}_{t} \subset \mathbb{R}_{>0}$ such that $\phi(x) \in X\left(\mathbf{a}_{0}\right) \phi^{\prime}\left(x^{\prime}\right) \in X\left(\mathbf{a}_{1}\right)$, and let $x_{t} \in X\left(\mathbf{a}_{t}\right)$ be a lifting of $\mathbf{a}_{t}$ such that $x_{0}=\phi(x)$. By Theorem 2.2. $T_{x_{0}}=T_{x_{1}}=T$, which shows that $x_{1}=\phi^{\prime}\left(x^{\prime}\right)$. Lifting $x_{t}$ to a path in $\mathcal{R}$, it follows that $\phi(\gamma)$ is in the same component as $\theta \circ \phi^{\prime}\left(\gamma^{\prime}\right)$ for some $\theta \in \mathrm{PGL}_{d}(\mathbb{R})$. But since $\epsilon_{\phi(\gamma)}=\epsilon_{\phi^{\prime}\left(\gamma^{\prime}\right)}$, $\theta$ must be in the connected component of the identity element. It follows that $\gamma$ and $\gamma^{\prime}$ are in the same connected component of $\mathcal{R}$.

Proof of Theorem 1.9. For (i), Lemmas 2.8, 2.9 and 2.10 imply that $\gamma \mapsto \mathcal{O}_{\gamma}$ descends to a bijection from the connected components of $\mathcal{R}$ to the $\operatorname{set} \operatorname{Orb}(\boldsymbol{j}, s)$. To see $\mathcal{R}$ is smooth it is enough to show that $\{\langle\gamma\rangle \mid \gamma \in \mathcal{R}\}$ is smooth. But this follows from the fact that $X$ is smooth at $x=\langle\gamma\rangle$, and the fibre $\mathrm{Wr}^{-1}(\mathrm{Wr}(x))$ is reduced. For any reflection $\theta \in \mathrm{PGL}_{2}(\mathbb{R})$ we have $\epsilon_{\gamma}=s \epsilon_{\theta \circ \gamma}$ and $\langle\gamma\rangle=\langle\theta \circ \gamma\rangle$; statement (ii) follows. It is enough to prove (iii) for the reflection $\left(\begin{array}{ll}0 & 1 \\ 1 & 0\end{array}\right) \in \mathrm{PGL}_{2}(\mathbb{R})$. Let $\hat{\gamma}=\left(\begin{array}{ll}0 & 1 \\ 1 & 0\end{array}\right) \gamma, \hat{x}=\langle\hat{\gamma}\rangle$. If $\phi \in M_{x}$, then $\hat{\phi}=\left(\begin{array}{ll}0 & 1 \\ 1 & 0\end{array}\right) \phi\left(\begin{array}{ll}0 & 1 \\ 1 & 0\end{array}\right) \in M_{\hat{x}}$. By Lemma 2.5 (i), $T_{\hat{\phi}(\hat{x})}=\boldsymbol{e}\left(T_{\phi(x)}\right)$. To compute the sign $\epsilon_{\hat{\phi}(\hat{\gamma})}$, note that there is a path $\gamma_{t}$ from $\hat{\phi}(\hat{\gamma})$ to $\left(\begin{array}{cc}-1 & 0 \\ 0 & 1\end{array}\right) \phi(\gamma)$ such that $\operatorname{Wr}\left(\gamma_{t} ; 0\right) \neq 0$ for all $t$. Thus the sign of $\hat{\phi}(\hat{\gamma})$ is the same as that of $\left(\begin{array}{rr}-1 & 0 \\ 0 & 1\end{array}\right) \phi(\gamma)$, which is $s^{\lfloor d / 2\rfloor} \epsilon_{\phi(\gamma)}$. Thus $\mathcal{O}_{\hat{\gamma}}=\left(\boldsymbol{e}, s^{\lfloor d / 2\rfloor}\right) \cdot \mathcal{O}_{\gamma}$, as required.

\section{The CASE OF AN INVOLUtion}

3.1. The generic cases. Suppose $\phi \in \mathrm{PGL}_{2}(\mathbb{C})$ is an involution (other than the identity element) and let $h(z) \in \mathbb{P}\left(\mathbb{C}_{N}[z]\right)$ be a $\phi$-fixed polynomial. We now consider the problem of counting the $\phi$-fixed points in $X(h(z))$. This is, of course, a special case of our more general problem. However, it is an interesting one because there are several interpretations of its various ingredients. For example, special choices of $h(z)$ and $\phi$ lead to different interpretations of the problem. Also, $\phi$ generates a group of order 2 , which we can think of as either the cyclic group $C_{2}$, or the dihedral group $D_{1}$. Hence all four of our theorems about fixed points (Theorems 1.7, 1.8, 1.10 and 1.11) will have something to say about this case. Unlike the general case, we can explicitly see how the different combinatorial objects that arise are related to each other. Finally, our results for involutions extend to the case where the roots of $h(z)$ are non-distinct.

Before we begin our investigations, we should note that there will be different cases to consider. Suppose $h(z)=\prod_{a_{i} \neq \infty}\left(z+a_{i}\right)$. For now we will assume that $\mathbf{a}=\left\{a_{1}, \ldots, a_{N}\right\}$ is a subset of $\mathbb{C P}^{1}$ such that $\phi(\mathbf{a})=\mathbf{a}$, and also that $X(\mathbf{a})$ is reduced. There are exactly two points in $\mathbb{C P}^{1}$ that are fixed by $\phi$. The number of non-fixed points of $\phi$ in a must be even. Thus we have three cases:

(1) $N$ is odd, and the set a contains exactly one $\phi$-fixed point of $\mathbb{C P}^{1}$.

(2) $N$ is even, and the set a contains no $\phi$-fixed points of $\mathbb{C P}^{1}$.

(3) $N$ is even, and the set a contains both $\phi$-fixed points $\mathbb{C P}^{1}$. 
When $\phi=\left(\begin{array}{ll}0 & 1 \\ 1 & 0\end{array}\right)$, these three cases correspond to the three generic types for $h(z)$ discussed in the introduction. They are fundamentally different, and we should expect to see different behaviours in each one. On the other hand, the answer only depends on which of the three cases we are in.

Lemma 3.1. For any a and $\phi$ as above, the number of $\phi$-fixed points in $X(\mathbf{a})$ depends only on the number of $\phi$-fixed elements of the set $\mathbf{a}$.

Proof. Let $\mathbb{P}\left(\mathbb{C}_{N}[z]\right)^{\phi}$ denote the $\phi$-fixed polynomials in $\mathbb{P}\left(\mathbb{C}_{N}[z]\right)$. Let $w_{1}, w_{2} \in$ $\mathbb{C P}^{1}$ denote the two $\phi$-fixed points. The $\phi$-fixed point scheme of $X$ is flat and finite over $\mathbb{P}\left(\mathbb{C}_{N}[z]\right)^{\phi}$. Hence the number of points in the reduced fibre $X(\mathbf{a})$ depends only on the component of $\mathbb{P}\left(\mathbb{C}_{N}[z]\right)^{\phi}$ that $h(z)$ lies in.

It is not hard to see that $\mathbb{P}\left(\mathbb{C}_{N}[z]\right)^{\phi}$ has exactly two components. If $N$ is odd, one component is the closure of the set of $h(z)$ identified with $\left\{\mathbf{a} \mid w_{1} \in \mathbf{a}\right\}$; the other is the closure of the set of all $h(z)$ identified with $\left\{\mathbf{a} \mid w_{2} \in \mathbf{a}\right\}$. These two components are isomorphic - any involution $\psi$ commuting with $\phi$ such that $\psi\left(w_{1}\right)=w_{2}$ gives an isomorphism - so we obtain the same answer for both components. If $N$ is even, the two components are non-isomorphic and correspond to cases (2) and (3).

We now describe a few of the different interpretations of the problem. Call $x \in X$ an even-odd point if $x$ has a basis $f_{1}(z), \ldots, f_{d}(z)$ where each $f_{i}(z)$ is either an even polynomial or an odd polynomial.

Proposition 3.2. Let $h(z)$ be an even or an odd polynomial $N$ with distinct roots. The even-odd points in $X(h(z))$ are exactly the $\left(\begin{array}{rr}-1 & 0 \\ 0 & 1\end{array}\right)$-fixed points.

Proof. An even-odd point in $X$ is clearly $\left(\begin{array}{cc}-1 & 0 \\ 0 & 1\end{array}\right)$-fixed. Conversely, any $\left(\begin{array}{cc}-1 & 0 \\ 0 & 1\end{array}\right)$-fixed subspace of $\mathbb{C}_{n-1}[z]$ has a basis of $\left(\begin{array}{cc}-1 & 0 \\ 0 & 1\end{array}\right)$-eigenvectors, and hence is an even-odd point.

Proposition 3.3. Let $h(z)$ be a real polynomial whose roots are all pure imaginary numbers. Then the real points in $X(h(z))$ are exactly the $\left(\begin{array}{cc}-1 & 0 \\ 0 & 1\end{array}\right)$-fixed points.

Proof. Let $\psi=\left(\begin{array}{cc}i & 0 \\ 0 & 1\end{array}\right) \in \mathrm{PGL}_{2}(\mathbb{C})$. We claim that $x \in X(h(z))$ is a real point if and only if $\psi(x)$ is an even-odd point. The result then follows from the fact that $\psi$ commutes with $\left(\begin{array}{cc}-1 & 0 \\ 0 & 1\end{array}\right)$.

The polynomial $\psi(h(z))=h(i z)$ has real roots, and hence by Theorem 1.2 every point $\psi(x) \in X(h(i z))$ is real. Suppose $\psi(x)$ has basis $f_{1}(z), \ldots, f_{d}(z)$, where each $f_{j}(z)$ is either an even polynomial or an odd polynomial. Then $x$ has basis $f_{1}(i z), \ldots, f_{d}(i z)$, each of which is either real or $i$ times a real polynomial. Hence $x$ is a real point.

Conversely, suppose $f_{1}(i z), \ldots, f_{d}(i z)$ are real polynomials that form a basis for $x \in X(h(z))$. Since $f_{1}(z), \ldots, f_{d}(z)$ span a real vector space $\psi(x) \in X(h(i z))$, so do their complex conjugates. Since $f_{j}(i z)$ is real, $\overline{f_{j}}(z)=f_{j}(-z)$, and so we deduce that $f_{1}(-z), \ldots, f_{d}(-z)$ span $\psi(x)$. Thus $\psi(x)$ is spanned by $f_{1}(z) \pm$ $f_{1}(-z), \ldots, f_{d}(z) \pm f_{d}(-z)$, which are odd or even polynomials, i.e. $\psi(x)$ is an even-odd point.

Proposition 3.4. Let $h(z)$ be a real polynomial with $N$ roots lying on a circle $O$ that is symmetrical with respect to the real axis. There is an involution $\phi \in \mathrm{PGL}_{2}(\mathbb{C})$ that restricts to complex conjugation on the circle $O$. The real points in $X(h(z))$ are the $\phi$-fixed points. 
Hence, by Lemma 3.1 the number of real points in $X(h(z))$ depends only on the number of real roots of $h(z)$. If $O=S^{1}$ is the unit circle, then this involution is $\phi=\left(\begin{array}{ll}0 & 1 \\ 1 & 0\end{array}\right)$.

Proof. There is a Möbius transformation $\psi \in \mathrm{PGL}_{2}(\mathbb{R})$ that sends the imaginary line to $O$. The involution $\phi=\psi\left(\begin{array}{rr}-1 & 0 \\ 0 & 1\end{array}\right) \psi^{-1}$ restricts to complex conjugation on $O$. Since $x \in X(h(z))$ is real and $\phi$-fixed if and only if $\psi^{-1}(x)$ is real and $\left(\begin{array}{cc}-1 & 0 \\ 0 & 1\end{array}\right)$-fixed, this result is equivalent to Proposition 3.3 .

Proposition 3.5. Suppose $N$ is even. Let $h(z)$ be a real, even polynomial with $N$ distinct non-real roots on the unit circle. Then the number of even-odd points in $X(h(z))$ is equal to the number of real points in $X(h(z))$.

Proof. We consider two involutions: $\left(\begin{array}{rr}-1 & 0 \\ 0 & 1\end{array}\right)$, whose fixed points in $\mathbb{C P}^{1}$ are $0, \infty$, and $\left(\begin{array}{ll}0 & 1 \\ 1 & 0\end{array}\right)$, whose fixed points in $\mathbb{C P}^{1}$ are \pm 1 . If $h(z)=\prod_{a_{i} \neq \infty}\left(z+a_{i}\right)$, then the set $\mathbf{a}$ is fixed by both $\left(\begin{array}{cc}-1 & 0 \\ 0 & 1\end{array}\right)$ and $\left(\begin{array}{ll}0 & 1 \\ 1 & 0\end{array}\right)$, and none of the elements of a are fixed by either. It follows from Lemma 3.1 that the number of $\left(\begin{array}{cc}-1 & 0 \\ 0 & 1\end{array}\right)$-fixed points in $X(h(z))$ is equal to the number of $\left(\begin{array}{ll}0 & 1 \\ 1 & 0\end{array}\right)$-fixed points in $X(h(z))$. But the former are even-odd points, and the latter are real points.

This last result has an interesting combinatorial interpretation. By Lemma 2.7 the real points in $X(h(z))$ correspond to $\boldsymbol{e}$-fixed tableaux in SYT $(\square)$. Note that $\boldsymbol{e}$ fixed tableaux are rotationally-invariant tableaux, so this statement is in agreement with both Theorems 1.10 and 1.11 (here we are considering the case where $r=1$ and where $h(z)$ is of type (2)). On the other hand, by Theorem 1.8, the odd-even points correspond to $j^{N / 2}$-fixed tableaux. Hence, from Proposition 3.5 we deduce:

Corollary 3.6. If $N$ is even, the number of $\boldsymbol{e}$-fixed tableaux in SYT( $\square)$ is equal to the number of $j^{N / 2}$-fixed tableaux in SYT $\left.\square\right)$.

The $r=2$ case of Theorem 1.7 asserts that the fixed points of an involution are also counted domino tableaux. We can prove the $r=2$ case of Theorem [1.7] using Lemma 2.7

Theorem $3.7(r=2$ case of Theorem 1.7). Let $h(z)$ be an even or an odd polynomial of degree $N$ or $N-1$, with distinct real roots. There is a unique $h(z)$-compatible Richardson variety $X_{\lambda / \mu}$, and the number of even-odd points in $X_{\lambda / \mu}(h(z))$ is the number of domino tableaux of shape $\lambda / \mu$.

Here the involution we use is $\left(\begin{array}{cc}-1 & 0 \\ 0 & 1\end{array}\right)$, which is the generator for $C_{2}$. The four possibilities for $\lambda / \mu$ are: $\square$ (if $h(z)$ is even and $N$ is even), $\square / 1$ (if $h(z)$ is odd and $N$ is odd), $1^{\vee}$ (if $h(z)$ is even and $N$ is odd), $1^{\vee} / 1$ (if $h(z)$ is odd and $N$ is even). The two cases where $N$ is odd give the same answer; here a contains either 0 or $\infty$, but not both. When $N$ is even and $\lambda / \mu=\square$, we are in the case where a contains neither 0 nor $\infty$. When $N$ is even and $\lambda / \mu=1^{\vee} / 1$, a contains both 0 and $\infty$. Hence these cases correspond exactly to the different possibilities for the number of $\left(\begin{array}{cc}-1 & 0 \\ 0 & 1\end{array}\right)$-fixed elements in a.

Proof. First, suppose that $\lambda / \mu=\square$. Then we can write $\mathbf{a}=\left\{a_{1}, \ldots, a_{N}\right\}$, where $0<a_{1}<a_{3}<\cdots<a_{N-1}<\infty$ and where $a_{2 k}=-a_{2 k-1}$ for all $k$. Let $T=T_{x}(\mathbf{a})$ be the real valued tableau associated to a point $x \in X(\mathbf{a})$. To compute $\left(\begin{array}{cc}-1 & 0 \\ 0 & 1\end{array}\right) T_{x}$, 
we write

$$
\left(\begin{array}{cc}
-1 & 0 \\
0 & 1
\end{array}\right)=\left(\begin{array}{ll}
1 & \varepsilon \\
0 & 1
\end{array}\right)\left(\begin{array}{cc}
-1 & 0 \\
0 & 1
\end{array}\right)\left(\begin{array}{ll}
1 & \varepsilon \\
0 & 1
\end{array}\right)
$$

where $\varepsilon$ is a small positive real number. Then $\left(\begin{array}{cc}1 & \varepsilon \\ 0 & 1\end{array}\right) \mathbf{a}=\left\{a_{1}+\varepsilon, \ldots, a_{N}+\varepsilon\right\}$. Note that

$$
\begin{aligned}
& a_{1} \prec a_{2} \prec a_{3} \prec a_{4} \prec \cdots \prec a_{N-1} \prec a_{N}, \\
& a_{2}+\varepsilon \prec a_{1}+\varepsilon \prec a_{4}+\varepsilon \prec a_{3}+\varepsilon \prec \cdots \prec a_{N}+\varepsilon \prec a_{N-1}+\varepsilon .
\end{aligned}
$$

By Theorem 2.3, $\left(\begin{array}{ll}1 & \varepsilon \\ 0 & 1\end{array}\right) T$ is obtained from $T$ by switching the positions of $a_{2 k-1}$ and $a_{2 k}=-a_{2 k-1}$ if they are in the same row and column, and then adding $\varepsilon$ to each of the entries. The action of $\left(\begin{array}{rr}-1 & 0 \\ 0 & 1\end{array}\right)$ on the result simply negates each of the entries by Lemma 2.5(i). Finally, acting by $\left(\begin{array}{ll}1 & \varepsilon \\ 0 & 1\end{array}\right)$ the second time does not change the $\preceq$-order of the entries, so we simply add $\varepsilon$ to each entry. The net result is that $\left(\begin{array}{cc}-1 & 0 \\ 0 & 1\end{array}\right) T$ is obtained from $T$ by switching the positions of $a_{2 k-1}$ and $a_{2 k}$ whenever they are not in the same row or column.

It follows that $\phi(x)=x$ if and only if the corresponding tableau $T_{x}(\mathbf{a})$ has the property that $a_{2 k-1}$ and $a_{2 k}$ are in the same row or column for all $k=1, \ldots, \frac{N}{2}$. These real valued tableaux are in bijection with domino tableaux of shape $\square$.

In the other three cases, $\mathbf{a}$ is of the form

$$
\begin{array}{cc}
\left\{0, a_{1}, \ldots, a_{N-1}\right\} & \text { if } \lambda / \mu=\square / 1, \\
\left\{a_{1}, \ldots, a_{N-1}, \infty\right\} & \text { if } \lambda / \mu=1^{\vee}, \\
\text { or } \quad\left\{0, a_{1}, \ldots, a_{N-2}, \infty\right\} & \text { if } \lambda / \mu=1^{\vee} / 1,
\end{array}
$$

where $0<a_{2 k-1}=-a_{2 k}<\infty$ for all $k$. A similar argument applies.

Corollary 3.8. If $N$ is even, the number of rotationally-invariant tableaux in SYT $\square$ ) is equal to the number of domino tableaux of shape $\square$.

A similar argument gives the $r=1$ case of Theorem 1.10 .

Theorem $3.9(r=1$ case of Theorem 1.10). Let $h(z)$ be a polynomial of degree $N$ whose roots are distinct real numbers invariant under $z \mapsto \frac{1}{z}$. If $h(1) \neq 0$ or $h(-1) \neq 0$, then the number of $\left(\begin{array}{ll}0 & 1 \\ 1 & 0\end{array}\right)$-fixed points in $X(h(z))$ is the number of rotationally-invariant tableaux in SYT $(\square)$. If $h(1)=h(-1)=0$, then the number of $\left(\begin{array}{ll}0 & 1 \\ 1 & 0\end{array}\right)$-fixed points in $X(h(z))$ is the number of rotationally-invariant tableaux in SYT $(\square)$, where $\frac{N}{2}$ and $\frac{N}{2}+1$ are in the same row or column.

Proof. Write $\mathbf{a}=\left\{a_{1}, \ldots, a_{N}\right\}$, where $a_{1} \prec a_{2} \prec \cdots \prec a_{N}$. Let $x \in X(\mathbf{a})$, with corresponding real valued tableau $T=T_{x}(\mathbf{a})$. It is enough to prove this when $h(z)$ is generic, so that $a_{i} \neq-a_{j}$ unless $\left\{a_{i}, a_{j}\right\}=\{1,-1\}$.

In the first case, when $h(1) \neq 0$ or $h(-1) \neq 0$, this implies that $\left|a_{1}\right|<\left|a_{2}\right|<$ $\cdots<\left|a_{N}\right|$. By Lemma 2.5(ii), $\left(\begin{array}{ll}0 & 1 \\ 1 & 0\end{array}\right) T=T^{\vee}$, and so $\left(\begin{array}{ll}0 & 1 \\ 1 & 0\end{array}\right) x=x$ if and only if the corresponding tableau is rotationally-invariant.

In the second case, when $h(1)=h(-1)=0$, we write

$$
\left(\begin{array}{ll}
0 & 1 \\
1 & 0
\end{array}\right)=\left(\begin{array}{cc}
1 & 0 \\
-\varepsilon & 1
\end{array}\right)\left(\begin{array}{ll}
0 & 1 \\
1 & 0
\end{array}\right)\left(\begin{array}{ll}
1 & \varepsilon \\
0 & 1
\end{array}\right)
$$

where $\varepsilon$ is a small positive real number. Arguing as in the proof of Theorem 3.7, we see that $\left(\begin{array}{ll}0 & 1 \\ 1 & 0\end{array}\right) T$ is obtained by rotating $T$ by $180^{\circ}$, replacing each entry $a_{k}$ by $\frac{1}{a_{k}}$, and switching the positions of 1 and -1 if and only if they are in the same row or 


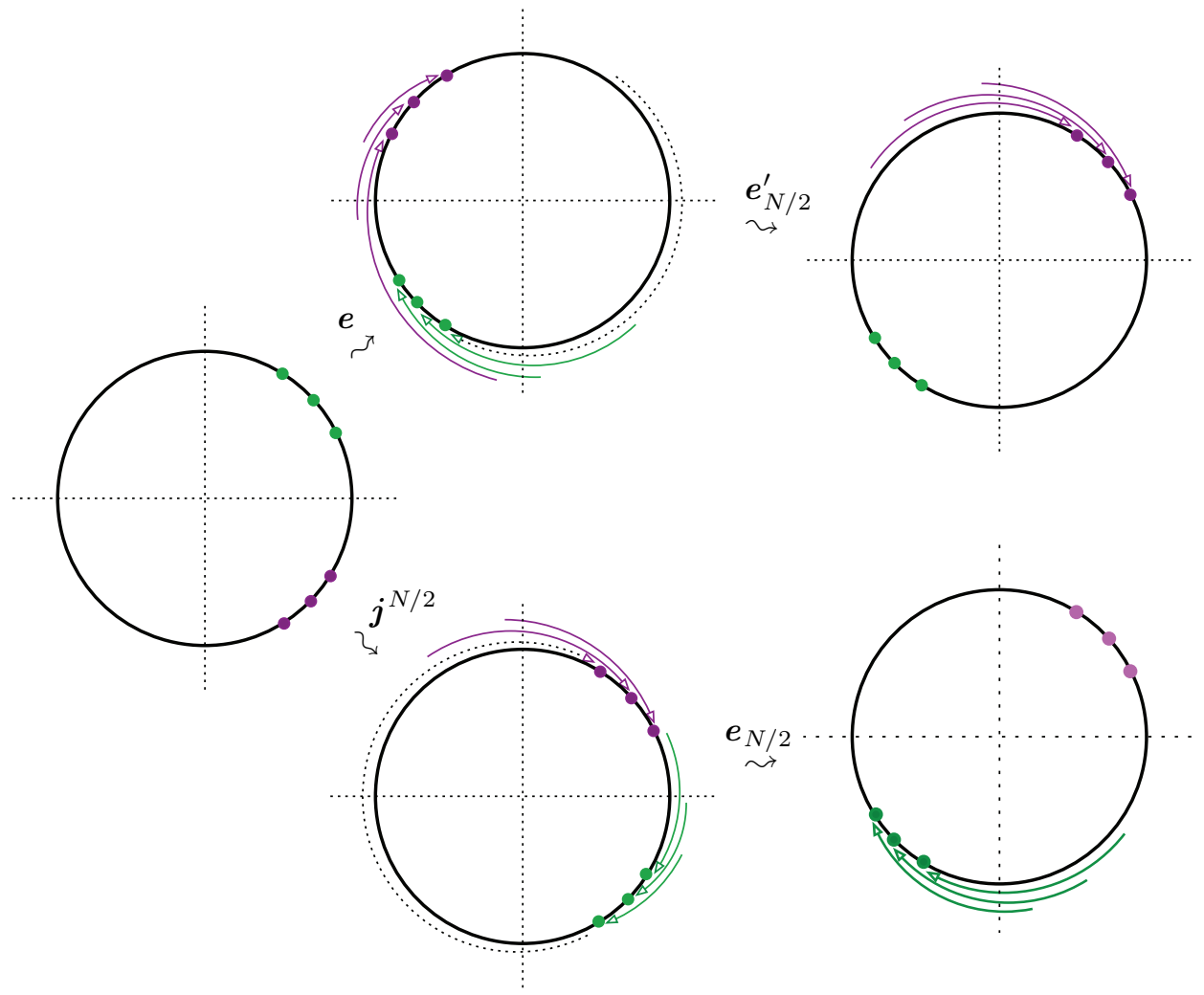

FIgURE 3.1. Homotopic paths that model $\boldsymbol{e}_{N / 2}^{\prime} \circ \boldsymbol{e}$ (top) and $\boldsymbol{e}_{N / 2} \circ$ $j^{N / 2}$ (bottom).

column. For $T$ to be invariant under this operation, we require rotation-invariance of $T_{x}$, and the entries $a_{N / 2}=1$ and $a_{(N / 2)+1}=-1$ of $T_{x}(\mathbf{a})$ must be in the same row or column.

Corollaries 3.6 and 3.8 can also be proved bijectively. The bijection between $\boldsymbol{e}$-fixed and $\boldsymbol{j}^{N / 2}$-fixed tableaux in SYT $(\square)$ is given by the involution $\boldsymbol{e}_{N / 2}$ : $\mathrm{SYT}(\square) \rightarrow \operatorname{SYT}(\square)$, which is the evacuation operator restricted to the entries $1, \ldots, \frac{N}{2}$.

Proposition 3.10. If $N$ is even, we have

$$
\boldsymbol{e} \circ \boldsymbol{e}_{N / 2}=\boldsymbol{e}_{N / 2} \circ \boldsymbol{j}^{N / 2} .
$$

Hence $\boldsymbol{e}_{N / 2}$ gives a bijection between $\boldsymbol{e}$-fixed and $\boldsymbol{j}^{N / 2}$-fixed tableaux in $\mathrm{SYT}(\square)$.

Proof. Let $\boldsymbol{e}_{N / 2}^{\prime}:=\boldsymbol{e} \circ \boldsymbol{e}_{N / 2} \circ \boldsymbol{e}$. For $T \in \mathrm{SYT}(\square)$, one can think of $\boldsymbol{e}_{N / 2}^{\prime}(T)$ as performing "reverse evacuation" on the largest $\frac{N}{2}$ entries of $T$. It is enough to show that $\boldsymbol{e}_{N / 2}^{\prime} \circ \boldsymbol{e}=\boldsymbol{e}_{N / 2} \circ \boldsymbol{j}^{N / 2}$. But as shown in Figure 3.1 the two sides of this equation are modelled by lifts of homotopic paths.

The bijection for Corollary 3.8 is a bit more involved. If $T$ is a domino tableau of shape $\square$, there are two entries labelled $k$ for each $k=1, \ldots, \frac{N}{2}$. One of these is 


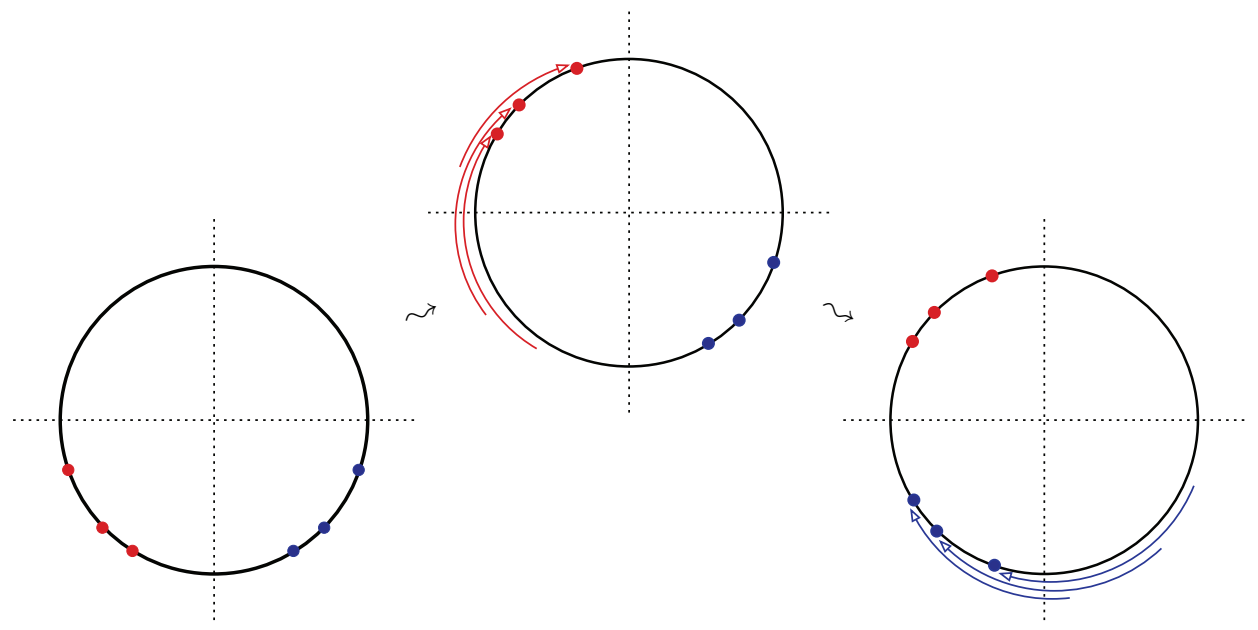

Figure 3.2. A path that models the bijection between domino tableaux of shape $\square$, and rotationally invariant tableaux of shape $\square$, when $N$ is even.

closer to the northwest corner; colour this entry blue and the other one red. With each $k$, starting at $k=\frac{N}{2}$ and descending to $k=1$, slide the red $k$ through the blue entries $k+1, \ldots, \frac{N}{2}$, and then replace the red $k$ by $k+\frac{N}{2}$. The result will be a standard Young tableau of shape $\square$. Finally, apply $\boldsymbol{e}_{N / 2}$ to this tableau, and denote the result $\varphi(T)$.

Proposition 3.11. The map $T \mapsto \varphi(T)$ gives a bijection between domino tableaux of shape $\square$ and rotationally-invariant tableaux in SYT( $\square)$.

Proof. Let $\mathbf{a}=\left\{a_{1}, \ldots, a_{N}\right\}$, where $0<a_{1}<a_{3}<\cdots<a_{N-1}<1$, and $a_{2 k}=$ $-a_{2 k-1}$ for $k=1, \ldots, \frac{N}{2}$. As we saw in the proof of Theorem 3.7, domino tableaux are in bijection with real valued tableaux of shape $\square$ with entries a, where $a_{2 k-1}$ and $a_{2 k}$ are in the same row or column, and these tableaux correspond to $\left(\begin{array}{cc}-1 & 0 \\ 0 & 1\end{array}\right)$ fixed points in $X(\mathbf{a})$. In this set-up, the map $\varphi$ is modelled by the path illustrated in Figure 3.2

Let

$$
\phi=\left(\begin{array}{cc}
\frac{1}{\sqrt{2}} & -\frac{1}{\sqrt{2}} \\
\frac{1}{\sqrt{2}} & \frac{1}{\sqrt{2}}
\end{array}\right) .
$$

When $\mathbb{R P}^{1}$ is drawn as a circle (as in Figure 2.1), $\phi \in \mathrm{PGL}_{2}(\mathbb{R})$ acts by rotating the circle $90^{\circ}$ clockwise. The path illustrated in Figure 3.2 is homotopic to a path that computes the action of $\phi$. Hence under the identifications above, the action of $\phi$ coincides with the map $\varphi$.

Let $T=T_{x}(\mathbf{a})$ be the real valued tableau corresponding to a point $x \in X(\mathbf{a})$. Note that $\phi\left(\begin{array}{rr}-1 & 0 \\ 0 & 1\end{array}\right) \phi^{-1}=\left(\begin{array}{ll}0 & 1 \\ 1 & 0\end{array}\right)$. Thus $x$ is a $\left(\begin{array}{rr}-1 & 0 \\ 0 & 1\end{array}\right)$-fixed point if and only if $\phi(x)$ is a $\left(\begin{array}{ll}0 & 1 \\ 1 & 0\end{array}\right)$-fixed point in $X(\phi(\mathbf{a}))$. By Lemma 2.5, the tableau corresponding to $\left(\begin{array}{ll}0 & 1 \\ 1 & 0\end{array}\right) \phi(x)$ is $\phi(T)^{\vee}$. Hence $\phi(T)$ corresponds to a $\left(\begin{array}{ll}0 & 1 \\ 1 & 0\end{array}\right)$-fixed point if and only if $\phi(T)=\phi(T)^{\vee}$. It follows that $T$ is identified with a domino tableau if and only if $\phi(T)$ is identified with a rotationally invariant tableau, and the result follows. 
Similar arguments can be used to establish explicit bijections between domino tableaux and rotationally-invariant tableaux (of the appropriate type) in cases (1) and (3). One can also show that the procedure used to define $\varphi(T)$ gives a bijection between domino tableau of shape $\lambda$ and the $\boldsymbol{e}$-fixed tableaux in $\operatorname{SYT}(\lambda)$ for any partition $\lambda$ of even size. We leave the details of these generalizations to the reader.

3.2. Involutions and Schubert intersections. The correspondence between points in $X(\mathbf{a})$ and tableaux can be extended to the case where $\mathbf{a}$ is a multiset of points in $\mathbb{R P}^{1}$. In this more general situation, the correspondence is no longer bijective. Nevertheless, we can still use it to obtain generalizations of Theorems 3.7 and 3.9 to the case where $\mathbf{a}$ is a multiset fixed by an involution $\phi \in \mathrm{PGL}_{2}(\mathbb{C})$. Although the problem of counting $\phi$-fixed points in $X(\mathbf{a})$ still makes some sense, the fibre $X(\mathbf{a})$ is not necessarily reduced when $\mathbf{a}$ is a multiset. We resolve this by refining our problem, and considering $\phi$-fixed points in an intersection of Schubert varieties.

For each $a \in \mathbb{C P}^{1}$, we define a flag in $\mathbb{C}_{n-1}[z]$ :

$$
F_{\bullet}(a):\{0\}=F_{0}(a) \subset F_{1}(a) \subset \cdots \subset F_{n-1}(a)=\mathbb{C}_{n-1}[z] .
$$

If $a \in \mathbb{C}$,

$$
F_{i}(a):=(z+a)^{n-i} \mathbb{C}[z] \cap \mathbb{C}_{n-1}[z] .
$$

In particular, $F_{\bullet}(0)$ is the flag $F_{\bullet}$ defined in Section 1.1. We define $F_{\bullet}(\infty)$ to be the flag $\widetilde{F}_{\bullet}$ (also defined in Section 1.1), which is equal to $\lim _{a \rightarrow \infty} F_{\bullet}(a)$. For every $\lambda \in \Lambda$, we have a Schubert variety in $X$ relative to the flag $F_{\bullet}(a)$ :

$$
\Omega^{\lambda}(a):=\left\{x \in X \mid \operatorname{dim}\left(x \cap F_{n-d-\lambda^{i}+i}(a)\right) \geq i, \text { for } i=1, \ldots, d\right\} .
$$

The codimension of $\Omega^{\lambda}(a)$ in $X$ is $|\lambda|$.

Proposition 3.12. The Wronskian $\operatorname{Wr}(x ; z)$ is divisible by $(z+a)^{k}$ if and only if $x \in \Omega^{\lambda}(a)$ for some partition $\lambda \vdash k$. Also, $x \in \Omega^{\mu}(\infty)$ for some $\mu \vdash(N-$ $\operatorname{deg} \operatorname{Wr}(x ; z))$.

Hence every point in $X(\mathbf{a})$ lies in some intersection of Schubert varieties relative to the flags $F_{\bullet}\left(a_{i}\right)$. (We will not discuss the multiplicities of such points here, but more information can be found in [20.) We are left to consider the problem of counting $\phi$-fixed points in an intersection

$$
\Omega^{\lambda_{1}}\left(a_{1}\right) \cap \cdots \cap \Omega^{\lambda_{s}}\left(a_{s}\right),
$$

where $a_{1}, \ldots, a_{s}$ are distinct, and $\left|\lambda_{1}\right|+\cdots+\left|\lambda_{s}\right|=N$. Mukhin, Tarasov and Varchenko proved that if $\mathbf{a}$ is real, such intersections of Schubert varieties are always transverse [19].

Theorem 3.13 (Mukhin-Tarasov-Varchenko). If $a_{1}, \ldots, a_{s} \in \mathbb{R P}^{1}$ are distinct real points, and $\lambda_{1}, \ldots, \lambda_{s} \in \Lambda$ are partitions with $\left|\lambda_{1}\right|+\cdots+\left|\lambda_{s}\right|=N$, then the intersection (3.1) is finite, transverse, and real.

Let us assume that $a_{1}, \ldots, a_{s}$ are real, and $a_{1} \prec a_{2} \prec \cdots \prec a_{s}$. Let $x$ be a point in the intersection (3.1). Instead of associating a unique tableau to the point $x$, we will associate a set of tableaux $\mathcal{T}_{x} \subset \mathrm{SYT}(\square)$. We do this by the reverse of the construction described in 22. Let a denote the multiset containing $a_{1}, \ldots, a_{s}$ with multiplicities $\left|\lambda_{1}\right|, \ldots,\left|\lambda_{s}\right|$, respectively, and let $x \in X(\mathbf{a})$. Consider a path $\mathbf{a}_{t}=\left\{\left(a_{1}\right)_{t} \preceq \cdots \preceq\left(a_{N}\right)_{t}\right\}, t \in[0,1]$, starting at $\mathbf{a}_{0}=\mathbf{a}$, and ending at a set 
$\mathbf{a}_{1}$. (Essentially we want to perturb a without changing the relative $\preceq$-order of the entries.) For any lifting to a path $x_{t} \in X\left(\mathbf{a}_{t}\right)$ with $x_{0}=x$, it is reasonable to think of $T_{x_{1}}$ as being a tableau associated to $x$. However, since $\mathbf{a}_{0}$ is a multiset, the fibre $X\left(\mathbf{a}_{0}\right)$ need not be reduced, and so there may be more than one such lifting. We define

$$
\mathcal{T}_{x}:=\left\{T_{x_{1}} \mid x_{t} \in X\left(\mathbf{a}_{t}\right), x_{0}=x\right\},
$$

the set of tableaux coming from all possible liftings of the path $\mathbf{a}_{t}$.

The sets $\mathcal{T}_{x}$ that arise in this way are characterized in terms of Haiman's dual equivalence relation [9]. We will not review all the relevant definitions here but instead refer the reader to [22, Section 2], where they may be found along with the proof of Theorem 3.14, below. Let $\boldsymbol{\lambda}$ denote the sequence of partitions $\left(\lambda_{1}, \ldots, \lambda_{s}\right)$. For $T \in \operatorname{SYT}(\square), k=1, \ldots, s$, let $T_{\boldsymbol{\lambda}}[k]$ be the subtableau of $T$ consisting of entries

$$
\left|\lambda_{1}\right|+\cdots+\left|\lambda_{k-1}\right|+1,\left|\lambda_{1}\right|+\cdots+\left|\lambda_{k-1}\right|+2, \ldots,\left|\lambda_{1}\right|+\cdots+\left|\lambda_{k}\right| .
$$

Write $T \sim_{\boldsymbol{\lambda}}^{*} T^{\prime}$ if $T_{\boldsymbol{\lambda}}[k]$ is dual equivalent to $T_{\boldsymbol{\lambda}}^{\prime}[k]$ for all $k=1, \ldots, s$. This is an equivalence relation on SYT( $\square)$. We will say that $T$ has type $\boldsymbol{\lambda}$ if $T_{\boldsymbol{\lambda}}[k]$ has rectification shape $\lambda_{k}$ for all $k$. Note that if $T$ has type $\boldsymbol{\lambda}$ and $T \sim_{\boldsymbol{\lambda}}^{*} T^{\prime}$, then $T^{\prime}$ also has type $\boldsymbol{\lambda}$.

Theorem 3.14. For every point $x \in X(\mathbf{a})$, the associated set of tableaux $\mathcal{T}_{x}$ is an equivalence class of the relation $\sim_{\boldsymbol{\lambda}}^{*}$. The point $x$ is in the intersection (3.1) if and only if $T$ has type $\boldsymbol{\lambda}$ for some (equivalently for every) $T \in \mathcal{T}_{x}$.

For $T \in \operatorname{SYT}(\square)$, switching the subtableaux $T_{\boldsymbol{\lambda}}[k]$ and $T_{\boldsymbol{\lambda}}[k+1]$ is done by using the following procedure (see e.g. [1]). First add $\left|\lambda_{k}\right|$ to each of the entries of $T_{\boldsymbol{\lambda}}[k]$, and subtract $\left|\lambda_{k}\right|$ from each of the entries of $T_{\boldsymbol{\lambda}}[k+1]$. Then, slide each of the boxes of $T_{\boldsymbol{\lambda}}[k]$, from largest entry to smallest, through $T_{\boldsymbol{\lambda}}[k+1]$. The result is a new tableau in SYT $(\square)$. Let $\operatorname{switch}(T, \mathbf{a})$ be the tableau obtained by switching $T_{\boldsymbol{\lambda}}[k]$ and $T_{\boldsymbol{\lambda}}[k+1]$ for each $k$ such that $a_{k}=-a_{k+1}$.

Theorem 3.15. Let $x$ be a point in the intersection (3.1).

(i) The point $\left(\begin{array}{cc}-1 & 0 \\ 0 & 1\end{array}\right) x$ lies in the intersection

$$
\Omega^{\lambda_{1}}\left(-a_{1}\right) \cap \cdots \cap \Omega^{\lambda_{s}}\left(-a_{s}\right),
$$

and the associated set of tableaux is $\left\{\operatorname{switch}(T, \mathbf{a}) \mid T \in \mathcal{T}_{x}\right\}$.

(ii) The point $\left(\begin{array}{ll}0 & 1 \\ 1 & 0\end{array}\right) x$ lies in the intersection

$$
\Omega^{\lambda_{1}}\left(a_{1}^{-1}\right) \cap \cdots \cap \Omega^{\lambda_{s}}\left(a_{s}^{-1}\right),
$$

and the associated set of tableaux is $\left\{\operatorname{switch}(T, \mathbf{a})^{\vee} \mid T \in \mathcal{T}_{x}\right\}$.

Here $T \mapsto T^{\vee}$ is the involution on SYT $(\square)$ which rotates a tableau by $180^{\circ}$ and replaces each entry $i$ by $N+1-i$.

Proof. We will prove (i), leaving (ii), which is similar, to the reader. Let $\hat{\mathbf{a}}=$ $\left(\begin{array}{cc}-1 & 0 \\ 0 & 1\end{array}\right)$ a and let $\hat{x}=\left(\begin{array}{cc}-1 & 0 \\ 0 & 1\end{array}\right) x$. Call a path $\mathbf{a}_{t}=\left\{\left(a_{1}\right)_{t}, \ldots,\left(a_{N}\right)_{t}\right\}$ short if $\left(a_{k}\right)_{t} \in$ $\mathbb{R P}^{1}$ is in some sufficiently small neighbourhood of $\left(a_{k}\right)_{0}$, for all $t \in[0,1], k=$ $1, \ldots, N$.

The definition (3.2) of $\mathcal{T}_{x}$ requires us to consider a "suitable" path $\mathbf{a}_{t}$, i.e. a path $\mathbf{a}_{t}=\left\{\left(a_{1}\right)_{t} \preceq \cdots \preceq\left(a_{N}\right)_{t}\right\}, t \in[0,1]$, where $\mathbf{a}_{0}=\mathbf{a}$ and $\mathbf{a}_{1}$ is a set. It is possible to choose this to be a short path, in which $\left(a_{k}\right)_{1}<\left(a_{k}\right)_{0}$ for all $k$, and 
we will assume that this is the case. This ensures that for any lifting $x_{t} \in X\left(\mathbf{a}_{t}\right)$, the tableau associated to $\left(\begin{array}{rr}-1 & 0 \\ 0 & 1\end{array}\right) x_{1}$ is just $T_{x_{1}}$ (by Lemma $2.5(\mathrm{i})$ ). Thus $\mathcal{T}_{x}$ is also obtained from all possible liftings of the path $\left(\begin{array}{cc}-1 & 0 \\ 0 & 1\end{array}\right) \mathbf{a}_{t}$, starting at $\hat{x}$.

Similarly, to obtain $\mathcal{T}_{\hat{x}}$ using (3.2) we need a suitable path $\hat{\mathbf{a}}_{t}=\left\{\left(\hat{a}_{1}\right)_{t}, \ldots,\left(\hat{a}_{N}\right)_{t}\right\}$, $t \in[0,1]$. Again, we assume this is a short path, and that $\left(\hat{a}_{k}\right)_{1}<\left(\hat{a}_{k}\right)_{0}$ for all $k$. Note that we have

$$
\left(\hat{a}_{k}\right)_{1}<\left(\hat{a}_{k}\right)_{0}=-\left(a_{k}\right)_{0}<-\left(a_{k}\right)_{1} \quad \text { for } k=1, \ldots, N .
$$

Now, any two short paths from $\hat{\mathbf{a}}$ to $\hat{\mathbf{a}}_{1}$ are homotopy equivalent to each other. In particular, the path $\hat{\mathbf{a}}_{t}$ is homotopy equivalent to the concatenation of $\left(\begin{array}{cc}-1 & 0 \\ 0 & 1\end{array}\right) \mathbf{a}_{t}$ with any short path of sets from $\left(\begin{array}{cc}-1 & 0 \\ 0 & 1\end{array}\right) \mathbf{a}_{1}$ to $\hat{\mathbf{a}}_{1}$. Hence we can compute $\mathcal{T}_{\hat{x}}$ by lifting this concatenation of paths, starting at $\hat{x}$. As noted above, the first part (lifting $\left(\begin{array}{cc}-1 & 0 \\ 0 & 1\end{array}\right) \mathbf{a}_{t}$ to a path starting at $\left.\hat{x}\right)$ gives us $\mathcal{T}_{x}$. It follows that the relationship between $\mathcal{T}_{x}$ and $\mathcal{T}_{\hat{x}}$ is described by lifting a short path from $\left(\begin{array}{cc}-1 & 0 \\ 0 & 1\end{array}\right) \mathbf{a}_{1}$ to $\hat{\mathbf{a}}_{1}$. From (3.5), we see that along such a path the points close to $a_{k}$ must change places (in the $\preceq$-order) with points close to $a_{k+1}$, whenever $a_{k+1}=-a_{k}$. By Theorem 2.3 , the effect of this on a tableau $T \in \mathcal{T}_{x}$ is to switch the subtableaux $T_{\boldsymbol{\lambda}}[k]$ and $T_{\boldsymbol{\lambda}}[k+1]$, and the result follows.

Theorem 3.15 gives us two ways of computing the number of fixed points of an involution $\phi \in \mathrm{PGL}_{2}(\mathbb{C})$ in the intersection (3.1). First note that for there to be any fixed points, the intersection itself must be $\phi$-invariant, which is to say we must have

$$
\left\{\left(\lambda_{1}, a_{1}\right), \ldots,\left(\lambda_{s}, a_{s}\right)\right\}=\left\{\left(\lambda_{1}, \phi\left(a_{1}\right)\right), \ldots,\left(\lambda_{s}, \phi\left(a_{s}\right)\right)\right\} .
$$

If we take $\phi=\left(\begin{array}{cc}-1 & 0 \\ 0 & 1\end{array}\right)$, the number of fixed points is the number of equivalence classes of $\sim_{\boldsymbol{\lambda}}^{*}$ of type $\boldsymbol{\lambda}$ that are invariant under $T \mapsto \operatorname{switch}(T, \mathbf{a})$. If we take $\phi=\left(\begin{array}{ll}0 & 1 \\ 1 & 0\end{array}\right)$, the number of fixed points is the number of equivalence classes of $\sim_{\boldsymbol{\lambda}}^{*}$ of type $\boldsymbol{\lambda}$ that are invariant under $T \mapsto \operatorname{switch}(T, \mathbf{a})^{\vee}$.

We note that the equivalence classes of $\sim_{\boldsymbol{\lambda}}^{*}$ are in bijection with sequences of Littlewood-Richardson tableaux, which makes it quite manageable to compute moderate sized examples by hand, by either method.

Example 3.16. Let $d=3, n=7$, and $\lambda=21$. Suppose $w_{1}, w_{2}$ are distinct nonreal complex numbers. We consider the problem of counting the number of real points in the intersection

$$
\Omega^{\lambda}\left(w_{1}\right) \cap \Omega^{\lambda}\left(\bar{w}_{1}\right) \cap \Omega^{\lambda}\left(w_{2}\right) \cap \Omega^{\lambda}\left(\bar{w}_{2}\right) .
$$

The points $w_{1}, \bar{w}_{1}, w_{2}, \bar{w}_{2}$ lie on a circle in $\mathbb{C P}^{1}$, so by Proposition 3.4 the real points in (3.6) are the fixed points of an involution $\phi \in \mathrm{PGL}_{2}(\mathbb{C})$. Hence this problem is equivalent to counting the $\left(\begin{array}{cc}-1 & 0 \\ 0 & 1\end{array}\right)$-fixed points in the intersection

$$
\Omega^{\lambda}\left(a_{1}\right) \cap \Omega^{\lambda}\left(-a_{1}\right) \cap \Omega^{\lambda}\left(a_{2}\right) \cap \Omega^{\lambda}\left(-a_{2}\right),
$$

where $0<a_{1}<a_{2}<\infty$. By Theorem 3.15)(i), the number of these points is the number of equivalence classes of $\sim_{\boldsymbol{\lambda}}^{*}$ of type $\boldsymbol{\lambda}=(\lambda, \lambda, \lambda, \lambda)$ that are invariant under $T \mapsto \operatorname{switch}(T, \mathbf{a})$.

There are eight equivalence classes of $\sim_{\boldsymbol{\lambda}}^{*}$ of type $\boldsymbol{\lambda}$; representatives of these classes are shown in Figure 3.3. To compute $T \mapsto \operatorname{switch}(T, \mathbf{a})$, we switch $T_{\boldsymbol{\lambda}}[1]$ with $T_{\boldsymbol{\lambda}}[2]$, and switch $T_{\boldsymbol{\lambda}}[3]$ with $T_{\boldsymbol{\lambda}}[4]$. The four tableaux in the top row are invariant under $T \mapsto \operatorname{switch}(T, \mathbf{a})$; therefore so are the equivalence classes they represent. For each tableaux $T$ in the bottom row, one can check that $T \nsim_{\lambda}^{*} \operatorname{switch}(T, \mathbf{a})$. Thus, 


\begin{tabular}{|c|c|c|c|}
\hline 1 & 2 & 7 & 8 \\
\hline 3 & 5 & 9 & 11 \\
\hline 4 & 6 & 10 & 12 \\
\hline
\end{tabular}

\begin{tabular}{|c|c|c|c|}
\hline 1 & 2 & 4 & 5 \\
\hline 3 & 6 & 8 & 11 \\
\hline 7 & 9 & 10 & 12 \\
\hline
\end{tabular}

\begin{tabular}{|c|c|c|c|}
\hline 1 & 2 & 5 & 7 \\
\hline 3 & 4 & 9 & 10 \\
\hline 6 & 8 & 11 & 12 \\
\hline
\end{tabular}

\begin{tabular}{|c|c|c|c|}
\hline 1 & 2 & 5 & 9 \\
\hline 3 & 6 & 7 & 10 \\
\hline 4 & 8 & 11 & 12 \\
\hline
\end{tabular}

\begin{tabular}{|c|c|c|c|}
\hline 1 & 2 & 4 & 5 \\
\hline 3 & 7 & 8 & 11 \\
\hline 6 & 9 & 10 & 12 \\
\hline
\end{tabular}

\begin{tabular}{|c|c|c|c|}
\hline 1 & 2 & 5 & 8 \\
\hline 3 & 4 & 6 & 11 \\
\hline 7 & 9 & 10 & 12 \\
\hline
\end{tabular}

\begin{tabular}{|c|c|c|c|}
\hline 1 & 2 & 5 & 9 \\
\hline 3 & 4 & 7 & 10 \\
\hline 6 & 8 & 11 & 12 \\
\hline
\end{tabular}

Figure 3.3. Eight tableaux that are representatives of the eight $\sim_{\boldsymbol{\lambda}}^{*}$ equivalence classes of type $\boldsymbol{\lambda}=(21,21,21,21)$, when $d=3$, $n=7$. The subtableaux $T_{\boldsymbol{\lambda}}[1], T_{\boldsymbol{\lambda}}[2], T_{\boldsymbol{\lambda}}[3]$ and $T_{\boldsymbol{\lambda}}[4]$ consist of entries $(1,2,3),(4,5,6),(7,8,9)$ and $(10,11,12)$, respectively.

exactly four of our equivalence classes are fixed by $T \mapsto \operatorname{switch}(T, \mathbf{a})$, and hence there are four real points in the intersection (3.6). This answer agrees with the experimental calculation in [10].

We can also obtain this answer by counting $\left(\begin{array}{ll}0 & 1 \\ 1 & 0\end{array}\right)$-fixed points in the intersection

$$
\Omega^{\lambda}\left(a_{1}\right) \cap \Omega^{\lambda}\left(a_{2}\right) \cap \Omega^{\lambda}\left(a_{2}^{-1}\right) \cap \Omega^{\lambda}\left(a_{1}^{-1}\right),
$$

where $\left|a_{1}\right|<\left|a_{2}\right|<1$. By Theorem 3.15(ii), these are counted by equivalence classes of $\sim_{\boldsymbol{\lambda}}^{*}$ of type $\boldsymbol{\lambda}$ that are invariant under $T \mapsto \operatorname{switch}\left(T^{\vee}, \mathbf{a}\right)=T^{\vee}$. The four tableaux on the left side of Figure 3.3 are representatives of these invariant classes.

\section{4. $C_{r}$-FIXed points of the Grassmannian}

4.1. Plücker coordinates. Virtually all of our calculations in the remaining sections of this paper will be done using the Plücker coordinates for the Grassmannian. Both the Richardson variety $X_{\lambda / \mu}$ and the fixed point set $X^{r}$ are characterized by the fact that certain Plücker coordinates are zero. In describing the latter, we begin to see how $r$-ribbons and $r$-ribbon tableaux fit into the picture.

There are several different ways to index Plücker coordinates: we will use $\Lambda$ as our indexing set. For $\lambda \in \Lambda$, set

$$
J(\lambda):=\left\{i-1+\lambda^{d+1-i} \mid 1 \leq i \leq d\right\} .
$$

Suppose $x \in X$ is the subspace spanned by polynomials $f_{1}(z), \ldots, f_{d}(z)$. Consider the $d \times n$ matrix $A_{i j}:=\left[z^{j}\right] f_{i}(z)$, whose entries are the coefficients of the polynomials $f_{i}(z)$. Our convention will be that the rows of $A_{i j}$ are indexed by $i=1, \ldots, d$, while the columns are indexed by $j=0, \ldots, n-1$. The Plücker coordinates of a point $x \in X$ are $\left[p_{\lambda}(x)\right]_{\lambda \in \Lambda}$, where $p_{\lambda}(x):=A_{J(\lambda)}$ is the maximal minor of $A$ with column set $J(\lambda)$. These are homogeneous coordinates: up to a scalar multiple, $\left[p_{\lambda}(x)\right]_{\lambda \in \Lambda}$ does not depend on the choice of basis.

We also define positive integer constants

$$
q_{\lambda}:=\prod_{1 \leq i<j \leq d}\left(j-i+\lambda^{d+1-j}-\lambda^{d+1-i}\right),
$$

for $\lambda \in \Lambda$. The Wronski map can be written explicitly in terms of the Plücker coordinates and the constants $q_{\lambda}$. 
Proposition 4.1 (See [20, Proposition 2.3]). For any point $x \in X$, the Wronskian $\mathrm{Wr}(x ; z)$ is given in terms of the Plücker coordinates of $x$ by

$$
\mathrm{Wr}(x ; z)=\sum_{\lambda \in \Lambda} q_{\lambda} p_{\lambda}(x) z^{|\lambda|} .
$$

Both sides of (4.1) are only defined up to a scalar multiple, hence this should rightly be interpreted as an equation in $\mathbb{P}\left(\mathbb{C}_{N}[z]\right)$.

We note a few useful results about the relationship between Plücker coordinates and Richardson varieties. The next two results are standard facts.

Proposition 4.2. Let $x \in X$. There is a unique maximal $\lambda \in \Lambda$ such that $p_{\lambda}(x) \neq$ 0 , and a unique minimal $\mu \in \Lambda$ such that $p_{\mu}(x) \neq 0$. For these partitions we have $x \in X_{\lambda / \mu}$.

Let $\Lambda_{\lambda / \mu}:=\{\nu \in \Lambda \mid \mu \subseteq \nu \subseteq \lambda\}$ denote the interval in the poset $\Lambda$ between $\mu$ and $\lambda$. The following theorem can be found in [11].

Theorem 4.3. As a subscheme of $X, X_{\lambda / \mu}$ is defined by the equation $\left\{p_{\nu}=0 \mid\right.$ $\left.\nu \notin \Lambda_{\lambda / \mu}\right\}$. In particular, for any point $x \in X_{\lambda / \mu}$, if $p_{\nu}(x) \neq 0$, then $\nu \in \Lambda_{\lambda / \mu}$.

Proposition 4.4. If $h(z) \in \mathbb{C}_{N}[z]$, and $X_{\lambda / \mu}$ is a compatible Richardson variety, then for any point in $x \in X_{\lambda / \mu}(h(z)):=X_{\lambda / \mu} \cap X(h(z))$ we have $p_{\mu}(x) \neq 0$ and $p_{\lambda}(x) \neq 0$.

Proof. Since $\operatorname{Wr}(x ; z)=h(z)$, taking coefficients of $z^{|\mu|}$ and $z^{|\lambda|}$ in (4.1), we have

$$
\sum_{|\nu|=|\mu|} q_{\nu} p_{\nu}(x)=\left[z^{|\mu|}\right] h(z) \quad \text { and } \quad \sum_{|\nu|=|\lambda|} q_{\nu} p_{\nu}(x)=\left[z^{|\lambda|}\right] h(z) .
$$

Since $X_{\lambda / \mu}$ is $h(z)$-compatible, the right hand sides are non-zero. Finally, for $p_{\nu}(x)$ to be non-zero we must have $\nu \in \Lambda_{\lambda / \mu}$; hence the first sum is just $q_{\mu} p_{\mu}(x)$, and the second is $q_{\lambda} p_{\lambda}(x)$. The result follows.

From these facts, we deduce Proposition 1.1.

Proof of Proposition 1.1. Let $x \in X(h(z))$. Let $\mu$ be the unique minimal partition such that $p_{\mu}(x) \neq 0$, and let $\lambda$ be the unique maximal partition such that $p_{\lambda}(x) \neq 0$. Then $x \in X_{\lambda / \mu}$. Moreover, from (4.1) we have $\left[x^{k}\right] h(z)=0$ for $k>|\lambda|$ and $\left[x^{|\lambda|}\right] h(z)=q_{\lambda} p_{\lambda}(x) \neq 0$. Thus $\operatorname{deg} h(z)=|\lambda|$, and similarly mindeg $h(z)=|\mu|$. Thus $x$ lies in some $h(z)$-compatible Richardson variety.

If $x$ were in two $h(z)$-compatible Richardson varieties, $x$ would lie in their intersection. This is a proper Richardson subvariety $X_{\lambda^{\prime} / \mu^{\prime}}$, where $\lambda^{\prime} \subsetneq \lambda$ or $\mu^{\prime} \supsetneq \mu$. But this contradicts Theorem 4.3. since $p_{\mu}(x) \neq 0$ and $p_{\lambda}(x) \neq 0$.

4.2. Components of $X^{r}$. The key to adapting the methods of [20] to the proof of Theorem 1.7 is an understanding of the components of $X^{r}$. We first show that these components are naturally indexed by two sets. The component of a point $x \in X^{r}$ can be indexed by an integer vector, determined by the eigenvalues of the $C_{r}$-action. It can also be indexed by an $r$-core in $\Lambda$, determined by the non-vanishing Plücker coordinates.

Let $\zeta=e^{\pi i / r}$, and let $c=\left(\begin{array}{cc}\zeta & 0 \\ 0 & \zeta^{-1}\end{array}\right)$. Viewed as an element of $\mathrm{PGL}_{2}(\mathbb{C}), c$ is a generator for the cyclic group $C_{r}$. However, viewed as an element of $\mathrm{SL}_{2}(\mathbb{C})$, it acts 
on the vector space $\mathbb{C}_{n-1}[z]$ as a semisimple operator. The monomials are a basis of eigenvectors: the action of $c$ on the polynomial $z^{k} \in \mathbb{C}_{n-1}[z]$ is given by

$$
c z^{k}=\zeta^{n-1-2 k} z^{k} .
$$

For $k=0, \ldots, r-1$, let $M_{k}$ denote the $\zeta^{n-1-2 k}$-eigenspace for the action of $c$ on $\mathbb{C}_{n-1}[z]$. Thus $M_{k}$ has a basis of monomials $\left\{z^{k}, z^{k+r}, \ldots, z^{k+\left(m_{k}-1\right) r}\right\}$, where $m_{k}:=\operatorname{dim} M_{k}=\left\lceil\frac{n-k}{r}\right\rceil$.

If $x \in X^{r}$, then the $c$ action on $\mathbb{C}_{n-1}[z]$ restricts to the invariant subspace $x$. Let $\operatorname{spec}^{r}(x)=\left(\operatorname{spec}_{0}^{r}(x), \ldots, \operatorname{spec}_{r-1}^{r}(x)\right)$, where $\operatorname{spec}_{k}^{r}(x)$ is the multiplicity of the eigenvalue $\zeta^{n-1-2 k}$ for the action of $c$ on $x$. Note that we have $\operatorname{spec}_{k}^{r}(x) \leq m_{k}$, and as the action is semisimple, $\operatorname{spec}_{0}^{r}(x)+\cdots+\operatorname{spec}_{r-1}^{r}(x)=d$. For any nonnegative integer vector $\mathbf{s}=\left(s_{0}, \ldots s_{r-1}\right)$, with $s_{0}+\cdots+s_{r-1}=d$ and $s_{k} \leq m_{k}$ for $k=0, \ldots, r-1$, let

$$
X^{\mathbf{s}}:=\left\{x \in X^{r} \mid \operatorname{spec}^{r}(x)=\mathbf{s}\right\} .
$$

Lemma 4.5. Let $\mathbf{s}$ be as above. Then $X^{\mathbf{s}}$ is naturally isomorphic to the product of Grassmannians $\prod_{k=0}^{r-1} \operatorname{Gr}\left(s_{k}, M_{k}\right)$.

In particular $X^{\mathbf{s}}$ is irreducible, which shows that the eigenvalues of the action of $c$ distinguish the components of $X^{r}$.

Proof. Each $x \in X^{r}$ decomposes as

$$
x=\bigoplus_{k=0}^{r-1}\left(x \cap M_{k}\right) .
$$

Now $x \in X^{\mathbf{s}}$ iff $\operatorname{dim}\left(x \cap M_{k}\right)=s_{k}, k=0, \ldots, r-1$, hence we must have $s_{k} \leq m_{k}$ for all $k$. In this case the $r$-tuple $\left(x \cap M_{0}, \ldots, x \cap M_{r-1}\right)$ is a point in $\operatorname{Gr}\left(s_{0}, M_{0}\right) \times$ $\cdots \times \operatorname{Gr}\left(s_{r-1}, M_{r-1}\right)$. Conversely any point $\left(\widetilde{x}_{0}, \ldots, \widetilde{x}_{r-1}\right)$ in this product of Grassmannians corresponds to the point $\widetilde{x}_{0} \oplus \widetilde{x}_{1} \oplus \cdots \oplus \widetilde{x}_{r-1} \in X^{\mathbf{s}}$.

A partition is called an $r$-core if its diagram does not have any hook lengths equal to $r$. Equivalently $\kappa$ is an $r$-core if there does not exist an $r$-ribbon tableau of shape $\kappa / \mu$ for any $\mu \subsetneq \kappa$. It is well known that if $\lambda$ is any partition, there is a unique $r$-core, $\operatorname{core}^{r}(\lambda) \subseteq \lambda$ (called the $r$-core of $\lambda$ ), such that there exists an $r$-ribbon tableau of shape $\lambda / \operatorname{core}^{r}(\lambda)$. It can be obtained by successively deleting $r$-ribbons from the shape $\lambda$ until it is no longer possible to do so. From this description it is not entirely obvious that $\operatorname{core}^{r}(\lambda)$ is well defined. This will be more evident from the alternate descriptions of $r$-cores that are given below.

Our next goal is to show that the components of $X^{r}$ are in bijection with the $r$-cores in $\Lambda$.

Example 4.6. Suppose $n=11, d=5$, and $r=3$, and consider the component $X^{(0,3,2)}$ of $X^{r}$. The points of this component are subspaces of $\mathbb{C}_{10}[z]$ spanned by 3 polynomials of the form $\alpha z+\beta z^{4}+\gamma z^{7}+\delta z^{10}$, and 2 polynomials of the form $\alpha z^{2}+\beta z^{5}+\gamma z^{8}$. The matrix $A_{i j}=\left[z^{j}\right] f_{i}(z)$, as in Section 4.1, is of the form

$$
\left(\begin{array}{lllllllllll}
0 & * & 0 & 0 & * & 0 & 0 & * & 0 & 0 & * \\
0 & * & 0 & 0 & * & 0 & 0 & * & 0 & 0 & * \\
0 & * & 0 & 0 & * & 0 & 0 & * & 0 & 0 & * \\
0 & 0 & * & 0 & 0 & * & 0 & 0 & * & 0 & 0 \\
0 & 0 & * & 0 & 0 & * & 0 & 0 & * & 0 & 0
\end{array}\right) .
$$



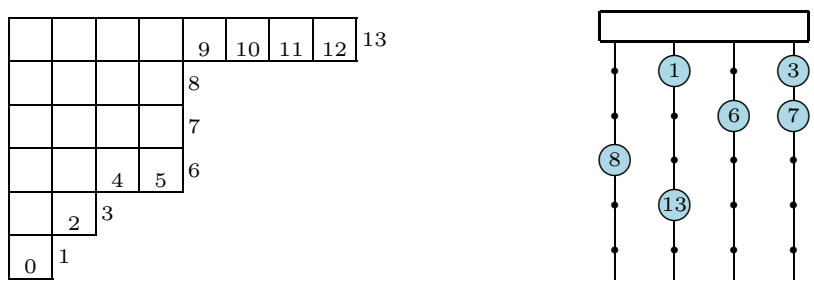

Figure 4.1. The partition 844421, and the corresponding 4abacus. Here $d=6$. The positions of the beads correspond to the up-steps along the boundary of the partition. The small dots in the abacus indicate positions without beads.

From this matrix, one can see that at most 12 of the 462 Plücker coordinates of a point $x \in X^{(0,3,2)}$ can be non-zero. The partitions indexing these Plücker coordinates all have 3 -core equal to 32211 .

Lemma 4.7. Let $X^{\mathbf{s}}$ be a component of $X^{r}$. There is a unique $r$-core in $\kappa \in \Lambda$ with the following property: for every $x \in X^{\mathbf{s}}$ and every partition $\lambda \in \Lambda$ whose $r$-core is not equal to $\kappa$, the Plücker coordinate $p_{\lambda}(x)$ is zero. Conversely, for every $r$-core $\kappa \in \Lambda$, there is a unique component $X^{\mathbf{s}} \subset X^{r}$ whose associated $r$-core is $\kappa$.

Thus the partitions indexing non-zero Plücker coordinates of a point $x \in X^{r}$ all have the same $r$-core, and this $r$-core determines the component of $x$. We will write $X^{r, \kappa}:=X^{\mathbf{s}}$ if $\kappa$ is the $r$-core corresponding to the component $X^{\mathbf{s}}$.

To prove Lemma 4.7, we use the correspondence between partitions and abacus diagrams. An $r$-abacus is an arrangement of $d$ beads inside an array with $r$ columns. The columns of this array are called runners, and are indexed $0, \ldots, r-1$. The possible positions of the beads are indexed by non-negative integers, where $0,1, \ldots, r-1$ are the positions in the first row (from left to right), $r, r+1, \ldots, 2 r-1$ are the positions in the second row, and so on. Thus the possible positions for beads on the $k^{\text {th }}$ runner are congruent to $k$ modulo $r$. The $r$-abacus associated to the partition $\lambda$ has beads in positions $J(\lambda)$. An example is given in Figure 4.1

It is not hard to show that deleting an $r$-ribbon from a partition corresponds to sliding a bead up one position in the corresponding $r$-abacus (see [12]). From this it follows that the $r$-core of a partition is determined by the number of beads on each runner of the $r$-abacus. A partition is an $r$-core if the corresponding $r$-abacus has its beads upwardly justified.

Proof. Let $x \in X^{r}$, and let $\lambda \in \Lambda$. Let $s_{k}$ be the number of beads on the $k^{\text {th }}$ runner of the $r$-abacus $J(\lambda), k=0, \ldots, r-1$. We claim that if $x \notin X^{\mathbf{s}}$, then $p_{\lambda}(x)=0$.

Suppose $x \in X^{\mathbf{s}^{\prime}}$, and $\mathbf{s}^{\prime} \neq \mathbf{s}$. Then for some $k, s_{k}<s_{k}^{\prime}$. Let $f_{1}(z), \ldots, f_{d}(z)$ be a basis of $c$-eigenvectors for $x$, where $f_{1}(z), \ldots, f_{s_{k}^{\prime}}(z) \in M_{k}$ are $\zeta^{n-1-2 k}$-eigenvectors. Then $p_{\lambda}(x)$ is given as the determinant of the minor of the matrix $A_{i j}$ as in Section 4.1 with columns $J(\lambda)$. Consider the first $s_{k}^{\prime}$ rows of this minor. There are at most $s_{k}$ non-zero entries in each row - they are in columns corresponding to the beads on the $k^{\text {th }}$ runner of the abacus. But since $s_{k}<s_{k}^{\prime}$ these rows cannot be linearly independent, and so the Plücker coordinate is $p_{\lambda}(x)$ zero.

It follows that if $x \in X^{\mathbf{s}}$, then the $p_{\lambda}(x)=0$ for every $\lambda \in \Lambda$ that does not have $s_{k}$ beads on the $k^{\text {th }}$ runner of the associated $r$-abacus. Let $\kappa$ be the $r$-core corresponding to $r$-abacus with $s_{k}$ upwardly justified beads on runner $k$. If $\operatorname{core}^{r}(\lambda) \neq \kappa$, 
then the $r$-abacus does not have the correct number of beads on each runner, and so $p_{\lambda}(x)=0$, as required.

Finally, let $\kappa \in \Lambda$ be an $r$-core. The argument above shows that there is at most one component $X^{r, \kappa}$ of $X^{r}$ associated to the $r$-core $\kappa$. To see that such a component is always non-empty, we note that there exists a point in $x \in X^{r}$ whose Plücker coordinates are $p_{\kappa}(x)=1, p_{\lambda}(x)=0$ for $\lambda \neq \kappa$. This $x$ is necessarily in $X^{r, \kappa}$.

Theorem 4.8. Let $x \in X$ and let $P_{x}:=\left\{\lambda \in \Lambda \mid p_{\lambda}(x) \neq 0\right\}$. Then $x \in X^{r, \kappa}$ if and only if all partitions in $P_{x}$ have $r$-core equal to $\kappa$.

Proof. We have already shown one direction in Lemma 4.7. It remains to show that if every partition in $P_{x}$ has $r$-core $\kappa$, then $x \in X^{r, \kappa}$. The action of $c$ on $X$ is given in Plücker coordinates by

$$
p_{\lambda}(c x)=\zeta^{-2|\lambda|} p_{\lambda}(x) \quad \text { for } \lambda \in \Lambda .
$$

If all partitions in $P_{x}$ have the same $r$-core $\kappa$, then for all $\lambda \in \Lambda$ we have either $\zeta^{2|\lambda|}=\zeta^{2|\kappa|}$ (if $\operatorname{core}^{r}(\lambda)=\kappa$ ) or $p_{\lambda}(x)=0$ (otherwise). Thus $\left[p_{\lambda}(c x)\right]_{\lambda \in \Lambda}$ is a scalar multiple of $\left[p_{\lambda}(x)\right]_{\lambda \in \Lambda}$, i.e. $c x=x$. Finally, $x \in X^{r, \kappa}$, since $p_{\lambda}(x) \neq 0$ for some partition with $\operatorname{core}^{r}(\lambda)=\kappa$.

Without any further work, we can already deduce a few facts about fixed points in the fibres of the Wronski map.

Corollary 4.9. Let $h(z)$ be a $C_{r}$-fixed polynomial, and let $X_{\lambda / \mu}$ be a compatible Richardson variety. Every point in $X_{\lambda / \mu}^{r}(h(z))$ lies in the same component of $X^{r}$.

Proof. Let $x \in X_{\lambda / \mu}^{r}(h(z))$. By Proposition 4.4, $p_{\mu}(x) \neq 0$, so $x$ lies in the component of $X^{r}$ corresponding to the $r$-core of $\mu$.

The next corollary is a special case of Theorem 1.7 .

Corollary 4.10. Let $h(z)$ be a $C_{r}$-fixed polynomial, and let $X_{\lambda / \mu}$ be a compatible Richardson variety. If $\lambda$ and $\mu$ do not have the same $r$-core, then the $X_{\lambda / \mu}^{r}(h(z))$ is empty. In this case there are no standard $r$-ribbon tableaux of shape $\lambda / \mu$.

Proof. If there exists a point $x \in X_{\lambda / \mu}^{r}(h(z))$, then $p_{\mu}(x) \neq 0$ and $p_{\lambda}(x) \neq 0$, so $\mu$ and $\lambda$ must have the same $r$-core. Since the $r$-core is obtained by deleting $r$ ribbons, if there exists an $r$-ribbon tableaux of shape $\lambda / \mu$, then $\mu$ and $\lambda$ have the same $r$-core.

4.3. Coordinate rings of components and their initial ideals. Let $\Lambda^{\mathbf{s}}$ denote the set of $r$-tuples of partitions $\left(\widetilde{\lambda}_{0}, \ldots, \widetilde{\lambda}_{r-1}\right)$, where $\widetilde{\lambda}_{k}: \widetilde{\lambda}_{k}^{0} \geq \cdots \geq \widetilde{\lambda}_{k}^{s_{k}}$ has at most $s_{k}$ parts and the largest part at most $m_{k}-s_{k}$.

The $r$-abacus $J(\lambda)$ can be encoded by a sequence of $r$ partitions in $\Lambda^{\mathbf{s}}$, where $s_{k}$ is the number of beads on the $k^{\text {th }}$ runner [7, 12, 27. We do this by encoding the sequence of beads on each runner into a partition in the standard way, where the positions of beads correspond to up-steps walking along the boundary of the partition. It is easier to state the reverse map, which sends

$$
\left(\widetilde{\lambda}_{0}, \ldots, \widetilde{\lambda}_{r-1}\right) \in \Lambda^{\mathbf{s}}
$$


to the abacus

$$
J(\lambda)=\left\{k+r\left(\widetilde{\lambda}_{k}^{s_{k}+1-i}+i-1\right) \mid i=1, \ldots, s_{k}, k=0, \ldots, r-1\right\} .
$$

The $r$-tuple $\left(\widetilde{\lambda}_{0}, \ldots, \widetilde{\lambda}_{r-1}\right)$ is called the $r$-quotient of $\lambda$.

Let $\kappa$ be the unique $r$-core whose abacus has $s_{k}$ beads on runner $k$. Let $\Lambda^{r, \kappa} \subset \Lambda$ be the set of all partitions with $r$-core $\kappa$. This is also the set of all partitions whose $r$-abacus has $s_{k}$ beads on runner $k$. Hence the $r$-quotient construction gives a bijection between $\Lambda^{r, \kappa}$ and $\Lambda^{\mathbf{s}}$. Moreover, sliding a bead up the $k^{\text {th }}$ runner of the abacus corresponds to deleting a box from the partition $\widetilde{\lambda}_{k}$ and to deleting an $r$-ribbon from $\lambda$. It follows that $\lambda \mapsto\left(\widetilde{\lambda}_{0}, \ldots, \widetilde{\lambda}_{r-1}\right)$ is an isomorphism of partially ordered sets $\Lambda^{r, \kappa} \simeq \Lambda^{\mathbf{s}}$. Since the latter is a distributive lattice, the former is too. (Note, however, it is not a sublattice of $\Lambda$ : the meet and join operations on $\Lambda^{r, \kappa}$ do not coincide with those of $\Lambda$.)

The elements of $\Lambda^{r, \kappa}$ index the non-zero Plücker coordinates on $X^{r, \kappa}=X^{\mathbf{s}}$, which by Lemma 4.5 is naturally identified with the product of $\prod_{k=0}^{r-1} \operatorname{Gr}\left(s_{k}, M_{k}\right)$. The $r$-quotient construction tells us how to identify the Plücker coordinates $p_{\lambda}$ with coordinates of the Segre embedding of this product of Grassmannians.

Let $\mathbb{C}[\mathbf{p}]$ denote the polynomial ring generated by indeterminates $\left\{p_{\lambda}\right\}_{\lambda \in \Lambda}$. The homogeneous coordinate ring of $X$ is $\mathbb{C}[X]=\mathbb{C}[\mathbf{p}] / I$, where $I$ is the Plücker ideal. $I$ is generated by quadratics, and describes all relations among the Plücker coordinates. Similarly, we write $\mathbb{C}\left[\operatorname{Gr}\left(s_{k}, M_{k}\right)\right]=\mathbb{C}\left[\mathbf{p}_{k}\right] / I_{k}$ for the homogeneous coordinate ring of $\operatorname{Gr}\left(s_{k}, M_{k}\right)$. The tensor product $\mathbb{C}\left[\operatorname{Gr}\left(s_{0}, M_{0}\right)\right] \otimes \cdots \otimes \mathbb{C}\left[\operatorname{Gr}\left(s_{r-1}, M_{r-1}\right)\right]$ is therefore the multihomogeneous ( $\mathbb{Z}^{r}$-graded) coordinate ring for $\prod_{k=0}^{r-1} \operatorname{Gr}\left(s_{k}, M_{k}\right)$. Abusing notation slightly, we will write the multihomogeneous ideal for this product as

$I_{0}+\cdots+I_{r-1}:=\left(I_{0} \otimes \mathbb{C}\left[\mathbf{p}_{1}\right] \otimes \cdots \otimes \mathbb{C}\left[\mathbf{p}_{r-1}\right]\right)+\cdots+\left(\mathbb{C}\left[\mathbf{p}_{0}\right] \otimes \cdots \otimes \mathbb{C}\left[\mathbf{p}_{r-2}\right] \otimes I_{r-1}\right)$.

There is a sign $\epsilon_{\lambda} \in\{ \pm 1\}$ associated to each partition $\lambda \in \Lambda$, which is easily obtained from the $r$-abacus. First let $\pi_{1}(\lambda), \ldots, \pi_{d}(\lambda)$ be the sequence obtained by listing the positions of the beads on runner 0 (in increasing order), followed by those on runner 1 , and so on. This list gives an ordering of the elements of $\widetilde{J}_{\lambda}$. We define $\epsilon_{\lambda}$ to be the sign of the permutation that puts them in increasing order.

Lemma 4.11. Consider the $\mathbb{C}$-algebra homomorphism $\Phi: \mathbb{C}[\mathbf{p}] \rightarrow \mathbb{C}\left[\mathbf{p}_{0}\right] \otimes \cdots \otimes$ $\mathbb{C}\left[\mathbf{p}_{r-1}\right]$ defined by

$$
\Phi\left(p_{\lambda}\right)= \begin{cases}\epsilon_{\lambda} p_{\widetilde{\lambda}_{0}} \otimes \cdots \otimes p_{\widetilde{\lambda}_{r-1}} & \text { if } \lambda \in \Lambda^{r, \kappa} \text { with r-quotient }\left(\widetilde{\lambda}_{0}, \ldots, \widetilde{\lambda}_{r-1}\right), \\ 0 & \text { if } \lambda \notin \Lambda^{r, \kappa} .\end{cases}
$$

The homogeneous ideal of $X^{r, \kappa}$ is $I^{r, \kappa}:=\Phi^{-1}\left(I_{0}+\cdots+I_{r-1}\right) \subset \mathbb{C}[\mathbf{p}]$.

Thus $\Phi$ descends to a homomorphism

$$
\hat{\Phi}: \mathbb{C}\left[X^{r, \kappa}\right] \rightarrow \mathbb{C}\left[\operatorname{Gr}\left(s_{0}, M_{0}\right)\right] \otimes \cdots \otimes \mathbb{C}\left[\operatorname{Gr}\left(s_{r-1}, M_{r-1}\right)\right]
$$

from the homogeneous coordinate ring of $X^{r, \kappa}$ to the multihomogeneous coordinate ring of $\prod_{k=0}^{r-1} \operatorname{Gr}\left(s_{k}, M_{k}\right)$. Hence (4.2) identifies the coordinates of $X^{r, \kappa}$ with the coordinates of the Segre embedding of $\prod_{k=0}^{r-1} \operatorname{Gr}\left(s_{k}, M_{k}\right)$.

Proof. Recall from the proof of Lemma 4.5 that the isomorphism $\prod_{k=0}^{r-1} \operatorname{Gr}\left(s_{k}, M_{k}\right)$ $\simeq X^{r, \kappa}$ is given by $\left(\widetilde{x}_{0}, \ldots, \widetilde{x}_{r-1}\right) \mapsto x=\widetilde{x}_{0} \oplus \cdots \oplus \widetilde{x}_{r-1}$, where $\widetilde{x}_{k}=x \cap M_{k}$. We 
must show that the Plücker coordinates of $x$ are (up to the specified sign) products of Plücker coordinates of $\widetilde{x}_{0}, \ldots, \widetilde{x}_{r-1}$.

Let $x \in X^{r, \kappa}=X^{\mathbf{s}}$ and let $f_{k 1}(z), \ldots, f_{k s_{k}}(z)$ be a basis for $\widetilde{x}_{k}=x \cap M_{k}$. The Plücker coordinates of $x \cap M_{k}$ are indexed by partitions $\widetilde{\lambda}_{k}$ with at most $s_{k}$ parts and its largest part $\leq m_{k}-s_{k}$. Let $\widetilde{A}_{i j}^{k}$ be the $s_{k} \times n$ matrix with entries $\widetilde{A}_{i j}^{k}=\left[z^{j}\right] f_{k i}(z)$. Then $p_{\widetilde{\lambda}_{k}}\left(\widetilde{x}_{k}\right)$ is the minor of $\widetilde{A}_{i j}^{k}=\left[z^{j}\right] f_{k i}(z)$ with columns specified by the positions of the beads on runner $k$ of the $r$-abacus $J(\lambda)$.

On the other hand $p_{\lambda}(x)$ is computed as the minor of the matrix

$$
A=\left(\begin{array}{c}
\widetilde{A}^{0} \\
\vdots \\
\widetilde{A}^{r-1}
\end{array}\right)
$$

with column set $J(\lambda)$. Suppose $\lambda \in \Lambda^{r, \kappa}$. If we take the columns of this minor in the order $\pi_{1}(\lambda), \ldots, \pi_{d}(\lambda)$, we see that this is a block diagonal matrix with determinant equal to

$$
p_{\widetilde{\lambda}_{0}}\left(\widetilde{x}_{0}\right) p_{\widetilde{\lambda}_{1}}\left(\widetilde{x}_{1}\right) \cdots p_{\widetilde{\lambda}_{r-1}}\left(\widetilde{x}_{r-1}\right) .
$$

But reordering the columns in this way changes the sign of the minor by $\epsilon_{\lambda}$, and thus we have

$$
p_{\lambda}(x)= \begin{cases}\epsilon_{\lambda} p_{\widetilde{\lambda}_{0}}\left(\widetilde{x}_{0}\right) p_{\widetilde{\lambda}_{1}}\left(\widetilde{x}_{1}\right) \cdots p_{\widetilde{\lambda}_{r-1}}\left(\widetilde{x}_{r-1}\right) & \text { if } \lambda \in \Lambda^{r, \kappa}, \\ 0 & \text { otherwise }\end{cases}
$$

Consider a Richardson variety $X_{\lambda / \mu}$, where $\lambda$ and $\mu$ both have an $r$-core equal to $\kappa$. Then the intersection $X_{\lambda / \mu}^{r, \kappa}:=X^{r, \kappa} \cap X_{\lambda / \mu}$ is non-empty. Write $I_{\lambda / \mu}^{r, \kappa} \subset \mathbb{C}[\mathbf{p}]$ for the homogeneous ideal of $X_{\lambda / \mu}^{r, \kappa}$. Also put $\Lambda_{\lambda / \mu}^{r, \kappa}:=\Lambda^{r, \kappa} \cap \Lambda_{\lambda / \mu}$.

Lemma 4.12. Suppose $\lambda$ and $\mu$ have r-quotients $\left(\widetilde{\lambda}_{0}, \ldots \widetilde{\lambda}_{r-1}\right)$ and $\left(\widetilde{\mu}_{0}, \ldots \widetilde{\mu}_{r-1}\right)$, respectively. Under the isomorphism $X^{r, \kappa} \cong \prod_{k=0}^{r-1} \operatorname{Gr}\left(s_{k}, M_{k}\right), X_{\lambda / \mu}^{r, \kappa}$ is identified with $\prod_{k=0}^{r-1} \widetilde{X}_{\widetilde{\lambda}_{k} / \widetilde{\mu}_{k}}$, where $\widetilde{X}_{\widetilde{\lambda}_{k} / \widetilde{\mu}_{k}}$ denotes the Richardson variety in $\operatorname{Gr}\left(s_{k}, M_{k}\right)$ corresponding to the skew shape $\widetilde{\lambda}_{k} / \widetilde{\mu}_{k}$.

Proof. For $k=0, \ldots, r-1$, let $I_{\widetilde{\lambda}_{k} / \widetilde{\mu}_{k}} \subset \mathbb{C}\left[\mathbf{p}_{k}\right]$ denote the homogeneous ideal of the Richardson variety $\widetilde{X}_{\widetilde{\lambda}_{k} / \widetilde{\mu}_{k}}$. By Theorem 4.3, this is generated by $I_{k}$, together with

$$
\left\{p_{\tau} \mid \tau \nsupseteq \widetilde{\mu}_{k} \text { or } \tau \nsubseteq \tilde{\lambda}_{k}\right\} \text {. }
$$

The ideal $I_{\lambda / \mu}^{r, \kappa}$ is generated by $I^{r, \kappa}$ and $\left\{p_{\nu} \mid \nu \notin \Lambda_{\lambda / \mu}\right\}$.

We must show that

$$
I_{\lambda / \mu}^{r, \kappa}=\Phi^{-1}\left(I_{\widetilde{\lambda}_{0} / \widetilde{\mu}_{0}}+\cdots+I_{\widetilde{\lambda}_{r-1} / \widetilde{\mu}_{r-1}}\right) .
$$

The right hand side of (4.4) is generated by $\Phi^{-1}\left(I_{0}+\cdots+I_{r-1}\right)=I^{r, \kappa}$ together with all

$$
\left\{p_{\nu} \mid \Phi\left(p_{\nu}\right) \in I_{\widetilde{\lambda}_{0} / \widetilde{\mu}_{0}}+\cdots+I_{\widetilde{\lambda}_{r-1} / \widetilde{\mu}_{r-1}}\right\} .
$$

By (4.2), $p_{\nu}$ is in this set if and only if $\nu \notin \Lambda^{r, \kappa}$ or $p_{\widetilde{\nu}_{k}}$ is in the set (4.3) for some $k$. The latter occurs when $\nu \in \Lambda^{r, \kappa} \backslash \Lambda_{\lambda / \mu}$. Thus the generators for the right hand side of (4.4) are the same as the generators for the left hand side, as required. 
We will need some facts about the initial ideal of $I^{r, \kappa}$ — and more generally the initial ideal of $I_{\lambda / \mu}^{r, \kappa}$ - for certain choices of weights on the Plücker coordinates.

Let $\mathbf{w}=\left(w_{\lambda}\right)_{\lambda \in \Lambda} \in \mathbb{Q}^{\Lambda}$ be a vector of rational numbers. The weight of a monomial $m(\mathbf{p})=c \prod_{\lambda \in \Lambda} p_{\lambda}^{k_{\lambda}} \in \mathbb{C}[\mathbf{p}]$ with respect to $\mathbf{w}$ is

$$
\mathrm{wt}_{\mathbf{w}}(m):=\sum_{\lambda \in \Lambda} w_{\lambda} k_{\lambda}
$$

If $g(\mathbf{p}) \in \mathbb{C}[\mathbf{p}]$, then the initial form of $g$ with respect to $\mathbf{w}$, denoted $\operatorname{In}_{\mathbf{w}}(g)$, is the sum of all monomial terms in $g$ for which the weight of the term is minimized. The initial ideal of an ideal $H \subset \mathbb{C}[\mathbf{p}]$ with respect to $\mathbf{w}$ is the ideal

$$
\operatorname{In}_{\mathbf{w}}(H):=\left\{\operatorname{In}_{\mathbf{w}}(g) \mid g \in H\right\} .
$$

In this context, the vector $\mathbf{w}$ is called a weight vector.

The weight vectors of interest to us will be constructed in the following way. We begin with an $r$-ribbon tableau $T$ of shape $\lambda / \mu$ and a sequence of rational numbers $b_{1}, \ldots, b_{\ell}$, where $r \ell=|\lambda / \mu|$.

Let $\square_{k}:=\left(m_{k}-s_{k}\right)^{s_{k}}$, so that $\left(\square_{0}, \ldots, \square_{r-1}\right)$ is the maximal element in the lattice $\Lambda^{\mathrm{s}}$. For each $i=1, \ldots, \ell$, the entries labelled $i$ in $T$ form a ribbon. This ribbon, under the $r$-quotient correspondence, is identified with a single box in some $\square_{k}$. Put the rational number $b_{i}$ in this box. We require our input data to be such that the following condition holds.

Condition 4.13. In the filling of $\square_{k}$, the entries are weakly decreasing along rows and down columns, and no $2 \times 2$ square has four equal entries.

In every case that we will consider, $b_{1}, \ldots, b_{\ell}$ will be a weakly decreasing sequence in which at most two of the $b_{i}$ are equal. This guarantees that Condition 4.13 will always be satisfied.

If not all the boxes of all $\square_{k}$ are filled, we fill the remaining boxes with rational numbers that are either less than $b_{1}$ or greater than $b_{\ell}$, so that Condition 4.13 is still satisfied. For each partition $\widetilde{\nu}_{k} \subset \square_{k}$, let $\widetilde{w}_{\widetilde{\nu}_{k}}$ be the sum of all entries in the filling of $\square_{k}$ that are outside the partition $\widetilde{\nu}_{k}$. The weight vector we consider will be

$$
w_{\nu}= \begin{cases}\sum_{k=0}^{r-1}\left(\widetilde{w}_{\widetilde{\nu}_{k}}-\widetilde{w}_{\widetilde{\lambda}_{k}}\right) & \text { if } \nu \in \Lambda^{r, \kappa} \text { with } r \text {-quotient }\left(\widetilde{\nu}_{0}, \ldots, \widetilde{\nu}_{r-1}\right), \\ 0 & \text { if } \nu \notin \Lambda^{r, \kappa}\end{cases}
$$

Example 4.14. Suppose $r=2, d=3, n=8$. Let us construct a weight vector $\mathbf{w}$ for the domino tableau

$$
T=\begin{array}{|l|l|l|l|l|}
\cline { 2 - 3 } & 2 & 3 & 4 & 4 \\
\hline 1 & 2 & 3 & 6 & \\
\hline 1 & 5 & 5 & 6 \\
\hline
\end{array}
$$

and the decreasing sequence $9,8,7,6,5,4$. We start by placing these numbers in the boxes of $\square_{0}$ and $\square_{1}$ corresponding to the ribbons in $T$ :

$$
\square_{0}: \begin{array}{|l|l|l|}
\hline 9 & 8 & \square \\
\hline
\end{array} \quad \square_{1}: \begin{array}{|l|l|}
\hline 7 & 6 \\
\hline 5 & 4 \\
\hline
\end{array} .
$$

For the empty box we have a choice (we are allowed to fill it with any number less than 4 ); here we will choose to fill it with a 1 . To determine any particular $w_{\nu}$, say 
for $\nu=322$, we shade the boxes corresponding to $\left(\widetilde{\nu}_{0}, \widetilde{\nu}_{1}\right)=(1,11)$,

$$
\square_{0}: \begin{array}{|l|l|l|}
\hline 9 & 8 & 1 \\
\hline
\end{array} \quad \square_{1}: \begin{array}{|l|l|}
\hline 7 & 6 \\
\hline 5 & 4 \\
\hline
\end{array},
$$

and sum the numbers outside of the shaded boxes, $8+1+6+4=19$. We do the same procedure for the partition $\lambda=544$; in this case the sum is 1 . Then $w_{322}$ is the difference of these two sums $w_{322}=19-1=18$. Note that $w_{\lambda}$ is always 0 , and the choice of numbers that fill the empty boxes does not affect $w_{\nu}$ if $\nu \in \Lambda_{\lambda / \mu}$.

For such a weight vector, the initial ideals of $I^{r, \kappa}$ and $I_{\lambda / \mu}^{r, \kappa}$ are described by the lattice structure of $\Lambda^{r, \kappa}$.

Lemma 4.15. For every weight vector $\mathbf{w}$ obtained from the process above, the initial ideal $\operatorname{In}_{\mathbf{w}}\left(I^{r, \kappa}\right)$ is generated by

$$
\left\{\left(\epsilon_{\nu \vee \sigma} p_{\nu \vee \sigma}\right)\left(\epsilon_{\nu \wedge \sigma} p_{\nu \wedge \sigma}\right)-\left(\epsilon_{\nu} p_{\nu}\right)\left(\epsilon_{\sigma} p_{\sigma}\right) \mid \nu, \sigma \in \Lambda^{r, \kappa}\right\} \cup\left\{p_{\nu} \mid \nu \notin \Lambda^{r, \kappa}\right\},
$$

and the initial ideal $\operatorname{In}_{\mathbf{w}}\left(I_{\lambda / \mu}^{r, \kappa}\right)$ is generated by

$$
\left\{\left(\epsilon_{\nu \vee \sigma} p_{\nu \vee \sigma}\right)\left(\epsilon_{\nu \wedge \sigma} p_{\nu \wedge \sigma}\right)-\left(\epsilon_{\nu} p_{\nu}\right)\left(\epsilon_{\sigma} p_{\sigma}\right) \mid \nu, \sigma \in \Lambda^{r, \kappa}\right\} \cup\left\{p_{\nu} \mid \nu \notin \Lambda_{\lambda / \mu}^{r, \kappa}\right\}
$$

Here $\wedge$ and $\vee$ denote the meet and join operations on the lattice $\Lambda^{r, \kappa}$.

Proof. It is enough to prove the second statement, since $I^{r, \kappa}=I_{\lambda / \mu}^{r, \kappa}$ in the case where $\mu=\kappa$ and $\lambda$ is the largest partition in $\Lambda^{r, \kappa}$.

As in the proof of Lemma 4.12, let $I_{\widetilde{\lambda}_{k} / \widetilde{\mu}_{k}} \subset \mathbb{C}\left[\mathbf{p}_{k}\right]$ denote the ideal of the Richardson variety $\widetilde{X}_{\widetilde{\lambda}_{k} / \widetilde{\mu}_{k}}$. Each of the vectors $\widetilde{\mathbf{w}}_{k}:=\left(\widetilde{w}_{\widetilde{\lambda}_{k}}\right)_{\widetilde{\lambda}_{k} \subset \square_{k}}, k=0, \ldots, r-1$, defines a weight vector for $\mathbb{C}\left[\mathbf{p}_{k}\right]$. It is well known (see [17, Theorem 14.16]) that for these weight vectors, if Condition 4.13 holds, the initial ideal $\operatorname{In}_{\widetilde{\mathbf{w}}_{k}}\left(I_{k}\right)$ is generated by the binomials

$$
\left\{p_{\tau \vee v} p_{\tau \wedge v}-p_{\tau} p_{v} \mid \tau, v \subseteq \square_{k}\right\}
$$

More generally, one can show (e.g. using [14, Theorem 7.1]) that $\operatorname{In}_{\widetilde{\mathbf{w}}_{k}}\left(I_{\widetilde{\lambda}_{k} / \widetilde{\mu}_{k}}\right)$ is generated by

$$
\left\{p_{\tau \vee v} p_{\tau \wedge v}-p_{\tau} p_{v} \mid \tau, v \subset \square_{k}\right\} \cup\left\{p_{\tau} \mid \tau \nsupseteq \widetilde{\mu}_{k} \text { or } \tau \nsubseteq \widetilde{\lambda}_{k}\right\} .
$$

The initial ideal of $I_{\widetilde{\lambda}_{0} / \widetilde{\mu}_{0}}+\cdots+I_{\widetilde{\lambda}_{r-1} / \widetilde{\mu}_{r-1}} \subset \mathbb{C}\left[\mathbf{p}_{0}\right] \otimes \cdots \otimes \mathbb{C}\left[\mathbf{p}_{r-1}\right]$ with respect to the weight vector $\widetilde{\mathbf{w}}:=\left(\widetilde{\mathbf{w}}_{0}, \ldots, \widetilde{\mathbf{w}}_{r-1}\right)$ is then the sum of these:

$$
\operatorname{In}_{\widetilde{\mathbf{w}}}\left(I_{\widetilde{\lambda}_{0} / \widetilde{\mu}_{0}}+\cdots+I_{\widetilde{\lambda}_{r-1} / \widetilde{\mu}_{r-1}}\right)=\operatorname{In}_{\widetilde{\mathbf{w}}_{0}}\left(I_{\widetilde{\lambda}_{0} / \widetilde{\mu}_{0}}\right)+\cdots+\operatorname{In}_{\widetilde{\mathbf{w}}_{r-1}}\left(I_{\widetilde{\lambda}_{r-1} / \widetilde{\mu}_{r-1}}\right) .
$$

This is a basic fact about sums of ideals in disjoint sets of variables, which follows readily from Buchburger's criterion.

Now, for any $g(\mathbf{p}) \in \mathbb{C}[\mathbf{p}], \Phi\left(\operatorname{In}_{\mathbf{w}}(g)\right)$ is either 0 or equal to $\operatorname{In}_{\widetilde{\mathbf{w}}}(\Phi(g))$. Moreover, any $\widetilde{\mathbf{w}}$-initial form in the image of $\Phi$ is the image of some $\mathbf{w}$-initial form in $\mathbb{C}[\mathbf{p}]$. It follows that

$$
\begin{aligned}
\operatorname{In}_{\mathbf{w}}\left(I_{\lambda / \mu}^{r, \kappa}\right) & =\operatorname{In}_{\mathbf{w}}\left(\Phi^{-1}\left(I_{\widetilde{\lambda}_{0} / \widetilde{\mu}_{0}}+\cdots+I_{\widetilde{\lambda}_{r-1} / \widetilde{\mu}_{r-1}}\right)\right) \\
& =\Phi^{-1}\left(\operatorname{In}_{\widetilde{\mathbf{w}}}\left(I_{\widetilde{\lambda}_{0} / \widetilde{\mu}_{0}}+\cdots+I_{\widetilde{\lambda}_{r-1} / \widetilde{\mu}_{r-1}}\right)\right) \\
& =\Phi^{-1}\left(\operatorname{In}_{\widetilde{\mathbf{w}}_{0}}\left(I_{\widetilde{\lambda}_{0} / \widetilde{\mu}_{0}}\right)+\cdots+\operatorname{In}_{\widetilde{\mathbf{w}}_{r-1}}\left(I_{\widetilde{\lambda}_{r-1} / \widetilde{\mu}_{r-1}}\right)\right) .
\end{aligned}
$$

Since each of the initial ideals $\operatorname{In}_{\widetilde{\mathbf{w}}_{k}}\left(I_{\widetilde{\lambda}_{k} / \widetilde{\mu}_{k}}\right)$ is generated in degree 1 and 2 , $\operatorname{In}_{\mathbf{w}}\left(I_{\lambda / \mu}^{r, \kappa}\right)$ is generated by the $\Phi$-preimage of the multihomogeneous elements of 
degree $(1,1, \ldots 1)$ and $(2,2, \ldots, 2)$ in $\operatorname{In}_{\widetilde{\mathbf{w}}_{0}}\left(I_{\widetilde{\lambda}_{0} / \widetilde{\mu}_{0}}\right)+\cdots+\operatorname{In}_{\widetilde{\mathbf{w}}_{r-1}}\left(I_{\widetilde{\lambda}_{r-1} / \widetilde{\mu}_{r-1}}\right)$. Thus $\operatorname{In}_{\mathbf{w}}\left(I_{\lambda / \mu}^{r, \kappa}\right)$ is generated by $\left\{p_{\lambda} \mid \lambda \notin \Lambda_{\lambda / \mu}^{r, \kappa}\right\}$ together with binomials of the form

$$
\left(\epsilon_{\nu^{\prime}} p_{\nu^{\prime}}\right)\left(\epsilon_{\sigma} p_{\sigma^{\prime}}\right)-\left(\epsilon_{\nu} p_{\nu}\right)\left(\epsilon_{\sigma} p_{\sigma}\right)
$$

where the $r$-quotients of $\nu, \sigma, \nu^{\prime}$ and $\sigma^{\prime}$ satisfy

$$
p_{{\widetilde{\nu^{\prime}}}_{k}} p_{{\widetilde{\sigma^{\prime}}}_{k}}-p_{\widetilde{\nu}_{k}} p_{\widetilde{\sigma}_{k}} \in \operatorname{In}_{\widetilde{\mathbf{w}}_{k}}\left(I_{k}\right) \quad \text { for } k=0, \ldots, r-1 .
$$

Among these are the binomials from (4.5), which arise when ${\widetilde{\nu^{\prime}}}_{k}=\widetilde{\nu}_{k} \vee \widetilde{\sigma}_{k}$ and ${\widetilde{\sigma^{\prime}}}_{k}=\widetilde{\nu}_{k} \wedge \widetilde{\sigma}_{k}$ for all $k$. On the other hand, each binomial (4.6) is in the ideal generated by (4.5). Indeed, (4.7) implies that $\nu^{\prime} \vee \sigma^{\prime}=\nu \vee \sigma$ and $\nu^{\prime} \wedge \sigma^{\prime}=\nu \wedge \sigma$, and so (4.6) can be written as

$$
\begin{aligned}
\left(\left(\epsilon_{\nu \vee \sigma} p_{\nu \vee \sigma}\right)\left(\epsilon_{\nu \wedge \sigma} p_{\nu \wedge \sigma}\right)-(\right. & \left.\left.\epsilon_{\nu} p_{\nu}\right)\left(\epsilon_{\sigma} p_{\sigma}\right)\right) \\
& -\left(\left(\epsilon_{\nu^{\prime} \vee \sigma^{\prime}} p_{\nu^{\prime} \vee \sigma^{\prime}}\right)\left(\epsilon_{\nu^{\prime} \wedge \sigma^{\prime}} p_{\nu^{\prime} \wedge \sigma^{\prime}}\right)-\left(\epsilon_{\nu^{\prime}} p_{\nu^{\prime}}\right)\left(\epsilon_{\sigma^{\prime}} p_{\sigma^{\prime}}\right)\right) .
\end{aligned}
$$

Thus $\operatorname{In}_{\mathbf{w}}\left(I_{\lambda / \mu}^{r, \kappa}\right)$ is generated by (4.5), as required.

\section{Fixed Points of the Fibre AND RIBbon tableauX}

5.1. Fibres of the Wronski map over a field of Puiseux series. Let $\mathbb{F}$ be an algebraically closed field of characteristic zero containing $\mathbb{C}$ as a subfield. Let $\mathbb{K}:=\mathbb{F}\{\{u\}\}=\bigcup_{n \geq 1} \mathbb{F}\left(\left(u^{\frac{1}{n}}\right)\right)$ be the field of Puiseux series over $\mathbb{F}$. This is also an algebraically closed field of characteristic zero, which contains $\mathbb{C}$ as a subfield. As noted in the introduction, it is enough to prove Theorem 1.7 working over any algebraically closed field, and we will do so working over the field $\mathbb{K}$. For most of our arguments it will suffice to consider $\mathbb{F}=\mathbb{C}$; however, for others we will take $\mathbb{F}=\mathbb{C}\{\{v\}\}$, the field of Puiseux series over $\mathbb{C}$.

From this point forward, all algebraic objects $\left(X, X_{\lambda / \mu}, \mathrm{Wr}\right.$, etc. $)$ will be considered over the field $\mathbb{K}$, rather than over $\mathbb{C}$. For example, $X:=\operatorname{Gr}\left(d, \mathbb{K}_{n-1}[z]\right)$ will be the Grassmannian of $d$-planes in the vector space $\mathbb{K}_{n-1}[z]$ over $\mathbb{K}$.

The advantage of working over $\mathbb{K}$ is that it is a complete valuation ring. If $g=c_{\ell} u^{\ell}+\sum_{k>\ell} c_{k} u^{k} \in \mathbb{K}^{\times}$, the valuation of $g$ is defined to be

$$
\operatorname{val}(g):=\ell \text {. }
$$

The leading term $\operatorname{LT}(g)$ and leading coefficient $\mathrm{LC}(g)$ are

$$
\operatorname{LT}(g):=c_{\ell} u^{\ell}, \quad \operatorname{LC}(g):=\left[u^{\ell}\right] g=c_{\ell} .
$$

Additionally, we set $\operatorname{val}(0):=+\infty, \operatorname{val}(\infty):=-\infty$ and $\operatorname{LT}(0):=0$. Let $\mathbb{K}_{+}:=\{g \in$ $\mathbb{K} \mid \operatorname{val}(g) \geq 0\}$.

Let $X_{\lambda / \mu}$ be a Richardson variety in $X$. Let $a_{1}, a_{2}, \ldots, a_{|\lambda / \mu|} \in \mathbb{K}^{\times}$be a non-zero Puiseux series, and put

$$
h(z)=z^{|\mu|} \prod_{i=1}^{|\lambda / \mu|}\left(z+a_{i}\right) .
$$

It will be convenient to adopt the convention that $a_{0}=0$, and $a_{|\lambda / \mu|+1}=\infty$. We will assume in what follows that the Plücker coordinates of a point $x \in X_{\lambda / \mu}(h(z))$ are normalized so that $p_{\lambda}(x)=1$.

In the case where $a_{1}, a_{2}, \ldots, a_{|\lambda / \mu|}$ have distinct valuations, the points in the fibre of $X_{\lambda / \mu}(h(z))$ can be identified with standard Young tableaux of shape $\lambda / \mu$. 
Lemma 5.1. Suppose that

$$
\operatorname{val}\left(a_{1}\right)>\operatorname{val}\left(a_{2}\right)>\cdots>\operatorname{val}\left(a_{|\lambda / \mu|}\right) .
$$

For every point $x \in X_{\lambda / \mu}(h(z))$, there is a unique chain of partitions $\mu=\lambda_{0} \subsetneq$ $\lambda_{1} \subsetneq \cdots \subsetneq \lambda_{|\lambda / \mu|}=\lambda$ with the property that

$$
\operatorname{val}\left(p_{\lambda_{k}}(x)\right)=\sum_{i=k+1}^{|\lambda / \mu|} \operatorname{val}\left(a_{i}\right) \quad \text { for } k=0, \ldots,|\lambda / \mu| .
$$

Moreover, for this chain we have

$$
L T\left(p_{\lambda_{k}}(x)\right)=\frac{q_{\lambda}}{q_{\lambda_{k}}} \prod_{i=k+1}^{|\lambda / \mu|} L T\left(a_{i}\right) \quad \text { for } k=0, \ldots,|\lambda / \mu| .
$$

The chain of partitions $\lambda_{0} \subsetneq \lambda_{1} \subsetneq \cdots \subsetneq \lambda$ can be identified with a standard Young tableaux in $T_{x} \in \operatorname{SYT}(\lambda / \mu)$, obtained by placing entry $k$ in the box $\lambda_{k} / \lambda_{k-1}$. $T_{x}$ is the tableau corresponding to the point $x \in X_{\lambda / \mu}(h(z))$.

For any shape $\nu \in \Lambda_{\lambda / \mu}$, let $T_{x}-\nu$ denote the set of entries in $T_{x}$ that are outside the shape $\nu$. Thus $T_{x}-\lambda_{k}=\{k+1, \ldots,|\lambda / \mu|\}$. The system (5.2) can therefore be rewritten as $\operatorname{val}\left(p_{\lambda_{k}}(x)\right)=\sum_{i \in\left(T_{x}-\lambda_{k}\right)} \operatorname{val}\left(a_{i}\right)$. In fact, when written this way, these equations hold for all Plücker coordinates of $X_{\lambda / \mu}$, not just those in the chain.

Lemma 5.2. Suppose that

$$
\operatorname{val}\left(a_{1}\right)>\operatorname{val}\left(a_{2}\right)>\cdots>\operatorname{val}\left(a_{|\lambda / \mu|}\right) .
$$

If $x \in X_{\lambda / \mu}(h(z))$, let $T=T_{x}$ be the corresponding tableau, and let $\lambda_{0} \subsetneq \lambda_{1} \subsetneq \cdots \subsetneq$ $\lambda$ be the associated chain of partitions. We have

$$
\operatorname{val}\left(p_{\nu}(x)\right)=\sum_{i \in(T-\nu)} \operatorname{val}\left(a_{i}\right) \quad \text { for } \nu \in \Lambda_{\lambda / \mu} .
$$

In particular, $p_{\lambda_{k}}(x)$ has the unique minimal valuation among all Plücker coordinates of $x$ indexed by partitions of size $\left|\lambda_{k}\right|$. That is, if $\nu \in \Lambda$ is any partition of size $\left|\lambda_{k}\right|$ but not equal to $\lambda_{k}$, we have $\operatorname{val}\left(p_{\nu}(x)\right)>\operatorname{val}\left(p_{\lambda_{k}}(x)\right)$.

Proof of Lemmas 5.1 and 5.2. This proof is little more than a trivial modification of the proof of [20, Corollary 4.4]. We sketch the main idea here, omitting several details that are the same.

Since the number of points in $X_{\lambda / \mu}(h(z))$ is exactly $|\mathrm{SYT}(\lambda / \mu)|$, it is enough to show that for each tableau $T \in \operatorname{SYT}(\lambda / \mu)$ encoding a chain of partitions $\mu=\lambda_{0} \subsetneq$ $\lambda_{1} \subsetneq \cdots \subsetneq \lambda$, there exists a point $x \in X_{\lambda / \mu}(h(z))$ such that (5.2), (5.3) and (5.4) hold.

To do this, we use [20, Theorem 4.2], which tells us how to obtain such a point from a solution to a certain system of equations. In our case, the system of equations we need to solve is

$$
q_{\lambda_{i}} \omega_{i+1} \cdots \omega_{|\lambda / \mu|}=q_{\lambda} \operatorname{LC}\left(a_{i+1}\right) \cdots \operatorname{LC}\left(a_{|\lambda / \mu|}\right), \quad i=1, \ldots,|\lambda / \mu|,
$$

in complex variables $\omega_{1}, \ldots, \omega_{|\lambda / \mu|}$. This has the unique solution

$$
\omega_{i}=\frac{q_{\lambda_{i}} \operatorname{LC}\left(a_{i}\right)}{q_{\lambda_{i-1}}} .
$$


According to [20, Theorem 4.2], every solution $\left(\omega_{1}, \ldots, \omega_{|\lambda / \mu|}\right)$ of (5.5) gives rise to a point $x \in X_{\lambda / \mu}(h(z))$ satisfying

$$
\operatorname{LT}\left(p_{\nu}(x)\right)=\left(\prod_{i \in(T-\nu)} \omega_{i}\right) u^{\left(\sum_{i \in(T-\nu)} \operatorname{val}\left(a_{i}\right)\right)} \quad \text { for } \nu \in \Lambda_{\lambda / \mu} .
$$

Taking valuations of both sides of (5.7), we see that $x$ satisfies (5.4). In the case where $\nu=\lambda_{k}$, we substitute the solution (5.6) into (5.7), giving

$$
\begin{aligned}
\operatorname{LT}\left(p_{\nu}(x)\right) & =\left(\prod_{i \in\left(T-\lambda_{k}\right)} \omega_{i}\right) u^{\left(\sum_{i \in\left(T-\lambda_{k}\right)} \operatorname{val}\left(a_{i}\right)\right)} \\
& =\left(\prod_{i=k+1}^{|\lambda / \mu|} \frac{q_{\lambda_{i}} \operatorname{LC}\left(a_{i}\right)}{q_{\lambda_{i-1}}}\right) u^{\left(\sum_{i=k+1}^{|\lambda / \mu|} \operatorname{val}\left(a_{i}\right)\right)} \\
& =\frac{q_{\lambda}}{q_{\lambda_{k}}} \prod_{i=k+1}^{|\lambda / \mu|} \operatorname{LC}\left(a_{i}\right) u^{\operatorname{val}\left(a_{i}\right)},
\end{aligned}
$$

which is equivalent to (5.3). This in turn implies (5.2).

Finally, we turn to the last statement of Lemma 5.2. Let $\nu \in \Lambda$ be any partition of size $\left|\lambda_{k}\right|$. If $\nu \in \Lambda_{\lambda / \mu}$, then since $\operatorname{val}\left(a_{1}\right)>\cdots>\operatorname{val}\left(a_{|\lambda / \mu|}\right)$,

$$
\operatorname{val}\left(p_{\nu}(x)\right)=\sum_{i \in(T-\nu)} \operatorname{val}\left(a_{i}\right) \geq \sum_{i=k+1}^{|\lambda / \mu|} \operatorname{val}\left(a_{i}\right)=\operatorname{val}\left(p_{\lambda_{k}}(x)\right) .
$$

Equality occurs if and only if $T-\nu=\{k+1, \ldots, N\}$, i.e. $\nu=\lambda_{k}$. If $\nu \notin \Lambda_{\lambda / \mu}$, then by Theorem $4.3 \operatorname{val}\left(p_{\nu}(x)\right)=+\infty>\operatorname{val}\left(p_{\lambda_{k}}(x)\right)$.

5.2. Beyond the generic case. For a polynomial $h(z)$ of the form (1.2) with coefficients in $\mathbb{K}$, the roots of $h(z)$ do not have distinct valuations. Thus Lemmas 5.1 and 5.2 cannot be used directly in the case of interest to us. Nevertheless, some parts of these lemmas still hold when the valuations of the roots are non-distinct.

Lemma 5.3. Suppose that

$$
\operatorname{val}\left(a_{1}\right) \geq \operatorname{val}\left(a_{2}\right) \geq \cdots \geq \operatorname{val}\left(a_{|\lambda / \mu|}\right) .
$$

There exists a chain of partitions $\mu=\lambda_{0} \subsetneq \lambda_{1} \subsetneq \cdots \subsetneq \lambda_{|\lambda / \mu|}=\lambda$ (not necessarily unique) encoded by a tableau $T \in \mathrm{SYT}(\lambda / \mu)$ with the following properties:

(1) For all $\nu \in \Lambda_{\lambda / \mu}$ we have

$$
\operatorname{val}\left(p_{\nu}(x)\right) \geq \sum_{i \in(T-\nu)} \operatorname{val}\left(a_{i}\right)
$$

Equality holds if the leading coefficients of $a_{1}, \ldots, a_{|\lambda / \mu|}$ are generic.

(2) If $\operatorname{val}\left(a_{k}\right)>\operatorname{val}\left(a_{k+1}\right)$, then (5.2) and (5.3) hold.

(3) If $\operatorname{val}\left(a_{k}\right)>\operatorname{val}\left(a_{k+1}\right)$, then $p_{\lambda_{k}}(x)$ has the unique minimal valuation among all Plücker coordinates of $x$ indexed by partitions of size $\left|\lambda_{k}\right|$. 
Proof. Fix a, and consider the family of finite $\mathbb{F}$-schemes over $\left(\mathbb{F}^{\times}\right)^{|\lambda / \mu|}$, whose fibre at a point $\mathbf{b}=\left(b_{1}, \ldots, b_{|\lambda / \mu|}\right)$ is $X_{\lambda / \mu}\left(h_{\mathbf{b}}(z)\right)$, where

$$
h_{\mathbf{b}}(z):=z^{|\mu|} \prod_{i=1}^{\mid \lambda / \mu}\left(z+a_{i} b_{i}\right) .
$$

For each component $C$ of the total space, there is a unique vector $\left(m_{\lambda}\right)_{\lambda \in \Lambda}$ such that $\operatorname{val}\left(p_{\lambda}(x)\right) \geq m_{\lambda}$ for all $x \in C$, with equality on a Zariski-dense subset of $C$.

We claim that there is a Zariski-dense subset $\mathcal{U} \subset\left(\mathbb{F}^{\times}\right)^{|\lambda / \mu|}$ such that if $\mathbf{b} \in \mathcal{U}$, then for any $x^{*} \in X_{\lambda / \mu}\left(h_{\mathbf{b}}(z)\right)$, (5.4) holds for the point $x^{*}$ and some tableau $T \in \operatorname{SYT}(\lambda / \mu)$. This shows that the vector $\left(m_{\lambda}\right)_{\lambda \in \Lambda}$ above is given by the right hand side of (5.4), which implies (1). This claim will also be used in the proof of (2).

To prove the claim, we work over $\mathbb{F}=\mathbb{C}\{\{v\}\}$, though still assuming $a_{1}, \ldots, a_{|\lambda / \mu|}$ $\in \mathbb{C}\{\{u\}\}$. Consider the set

$$
\mathcal{U}=\left\{\left(v^{\gamma_{1}}, \ldots, v^{\gamma_{|\lambda / \mu|}}\right) \mid \gamma_{k} \in \mathbb{Z}, \gamma_{1}>\cdots>\gamma_{|\lambda / \mu|}\right\}
$$

which is Zariski-dense in $\mathbb{F}^{|\lambda / \mu|}$. Fix $\mathbf{b}=\left(v^{\gamma_{1}}, \ldots, v^{\gamma_{|\lambda / \mu|}}\right) \in \mathcal{U}$, and let $\varepsilon>0$ be a small positive rational number. Put

$$
\hat{h}(z):=z^{|\mu|} \prod_{i=1}^{\mid \lambda / \mu}\left(z+\hat{a}_{i}\right),
$$

where $\hat{a}_{k}=a_{k} v^{\gamma_{k}} u^{\varepsilon \gamma_{k}}$. Since $\varepsilon$ is assumed to be small, we have $\operatorname{val}\left(\hat{a}_{1}\right)>\cdots>$ $\operatorname{val}\left(\hat{a}_{|\lambda / \mu|}\right)$, so for any point $\hat{x} \in X_{\lambda / \mu}(\hat{h}(z))$, (5.4) holds. In particular, there is a tableau $T$ such that

$$
\begin{aligned}
\operatorname{val}\left(p_{\nu}(\hat{x})\right) & =\sum_{i \in(T-\nu)} \operatorname{val}\left(\hat{a}_{i}\right) \\
& =\sum_{i \in(T-\nu)} \operatorname{val}\left(a_{i}\right)+O(\varepsilon)
\end{aligned}
$$

for $\nu \in \Lambda_{\lambda / \mu}$.

Now consider the substitution $\Sigma: v \mapsto v u^{-\varepsilon}$. Although this does not give a well-defined transformation from $\mathbb{K}$ to itself, we do have $\Sigma\left(p_{\nu}(\hat{x})\right) \in \mathbb{K}$, because the valuations of the Plücker coordinates of $\hat{x}$ are uniformly bounded below for all positive $\varepsilon$ near 0 . Thus we obtain a point

$$
x^{*}:=\Sigma(\hat{x}) \in X_{\lambda / \mu}(\Sigma(\hat{h}(z)))=X_{\lambda / \mu}\left(h_{\mathbf{b}}(z)\right),
$$

and every point in $X\left(h_{\mathbf{b}}(z)\right)$ is of this form. The Plücker coordinates of $x^{*}$ satisfy

$$
\begin{aligned}
\operatorname{val}\left(p_{\nu}\left(x^{*}\right)\right) & =\operatorname{val}\left(p_{\nu}(\hat{x})\right)+O(\varepsilon) \\
& =\sum_{i \in(T-\nu)} \operatorname{val}\left(a_{i}\right)+O(\varepsilon)
\end{aligned}
$$

for $\nu \in \Lambda_{\lambda / \mu}$. Since $\varepsilon$ can be made arbitrarily small, we see that (5.4) holds for the point $x^{*}$.

Next we prove (2). Since the right hand side of (5.3) is a continuous nowherevanishing function of $\operatorname{LT}\left(a_{1}\right), \ldots, \operatorname{LT}\left(a_{|\lambda / \mu|}\right)$, it is enough to prove this under the assumption that the leading coefficients $\operatorname{LC}\left(a_{1}\right), \ldots, \operatorname{LC}\left(a_{|\lambda / \mu|}\right)$ are generic. Our claim shows that in this case (5.4) holds for $x$ and some tableau $T \in \operatorname{SYT}(\lambda / \mu)$. 
Suppose $T$ encodes the chain of partitions $\mu=\lambda_{0} \subsetneq \lambda_{1} \subsetneq \cdots \subsetneq \lambda_{|\lambda / \mu|}=\lambda$. As in the proof of Lemma [5.2. it follows that when $\operatorname{val}\left(a_{k}\right)>\operatorname{val}\left(a_{k+1}\right), p_{\lambda_{k}}(x)$ has the unique minimal valuation among all Plücker coordinates of $x$ indexed by partitions of size $\left|\lambda_{k}\right|$.

Now $h(z)=\operatorname{Wr}(x ; z)$, which we can rewrite using (4.1) as

$$
q_{\lambda} z^{|\mu|} \prod_{k=1}^{|\lambda / \mu|}\left(z+a_{k}\right)=\sum_{\nu \in \Lambda} q_{\nu} p_{\nu}(x) z^{|\nu|} .
$$

(Since the Plücker coordinates are assumed to be normalized so that $p_{\lambda}(x)=1$, we need the factor $q_{\lambda}$ on the left hand side to ensure that the coefficients of $z^{|\lambda|}$ agree on both sides.) Taking the coefficient of $z^{|\mu|+k}$ on both sides, we see that

$$
q_{\lambda} e_{|\lambda / \mu|-k}\left(a_{1}, \ldots, a_{|\lambda / \mu|}\right)=\sum_{\nu \vdash \mid(\mu \mid+k)} q_{\nu} p_{\nu}(x),
$$

where $e_{i}$ denotes the $i^{\text {th }}$ elementary symmetric function. Now take the leading term of both sides. In the case where $\operatorname{val}\left(a_{k}\right)>\operatorname{val}\left(a_{k+1}\right)$, the leading term of the right hand side is

$$
q_{\lambda_{k}} \operatorname{LT}\left(p_{\lambda_{k}}\right),
$$

as all other monomials in the sum have strictly larger valuation. By similar reasoning, the leading term of the left hand side is

$$
q_{\lambda} \operatorname{LT}\left(a_{k+1}\right) \cdots \operatorname{LT}\left(a_{|\lambda / \mu|}\right) .
$$

Equating (5.9) and (5.10), we obtain (5.3). Taking valuations of both sides gives (5.2).

Finally, we use the last argument in the proof of Lemma $\$ 5.2$ once more to deduce (3) from (1) and (2).

The chain of partitions in Lemma 5.3 is not necessarily uniquely determined by the point $x$. However, if $0=k_{0}<k_{1}<\cdots<k_{\ell-1}<k_{\ell}=|\lambda / \mu|$ are the indices for which $\operatorname{val}\left(a_{k_{i}}\right)>\operatorname{val}\left(a_{k_{i}+1}\right)$, then the subchain

$$
\mu=\lambda_{0} \subsetneq \lambda_{k_{1}} \subsetneq \cdots \subsetneq \lambda_{k_{\ell}}=\lambda
$$

is characterized by property (3) in Lemma 5.3 . Hence this particular subchain of partitions is uniquely determined by $x$. We can encode this subchain into a tableau, again denoted $T_{x}$, by placing entry $i$ in each of the boxes of $\lambda_{k_{i}} / \lambda_{k_{i}-1}$.

In this way we can associate a tableau to every point $x \in X$. This is not always an interesting tableau. For example, if $h(z) \in \mathbb{F}[z]$, then all roots have the same valuation, and so $T_{x}$ is the tableau in which every entry is 1 . In particular, the reader should note that this definition of $T_{x}$ does not agree with the definition given in Section 2, We now show that in the case of interest to us, when $x$ is a generic $C^{r}$-fixed point, this tableau is a standard $r$-ribbon tableau.

Lemma 5.4. Suppose that $h(z)$ is a $C_{r}$-fixed polynomial of the form (1.2), where

$$
\operatorname{val}\left(h_{1}\right)>\operatorname{val}\left(h_{2}\right)>\cdots>\operatorname{val}\left(h_{\ell}\right) .
$$

Let $X_{\lambda / \mu}$ be an $h(z)$-compatible Richardson variety. If $x \in X_{\lambda / \mu}^{r}(h(z))$, then the corresponding tableau $T_{x}$ is a standard $r$-ribbon tableau of shape $\lambda / \mu$. 
Proof. Rewrite $h(z)$ in the form (5.1). Since each binomial $\left(z^{r}+h_{i}\right)$ has $r$ roots, each with valuation $\frac{1}{r} \operatorname{val}\left(h_{i}\right)$, we may assume that

$$
\begin{gathered}
\operatorname{val}\left(a_{1}\right)=\cdots=\operatorname{val}\left(a_{r}\right)>\operatorname{val}\left(a_{r+1}\right)=\cdots=\operatorname{val}\left(a_{2 r}\right) \\
>\operatorname{val}\left(a_{2 r+1}\right)=\cdots=\operatorname{val}\left(a_{3 r}\right) \\
\vdots \\
>\operatorname{val}\left(a_{r \ell-r+1}\right)=\cdots=\operatorname{val}\left(a_{r \ell}\right) .
\end{gathered}
$$

Thus the tableau $T_{x}$ has entries $1,2, \ldots, \ell$, each appearing $r$ times.

Let $\mu=\lambda_{0} \subsetneq \lambda_{1} \subsetneq \cdots \subsetneq \lambda_{\ell}=\lambda$ be the chain of partitions associated to $T_{x}$. By Lemma $5.3(2)$, each of the Plücker coordinates $p_{\lambda_{k}}(x)$ satisfies (5.3) and hence is non-zero, for $k=0, \ldots, \ell$. By Lemma 4.7, this implies that each of the partitions in the chain have the same $r$-core. In particular, $\lambda_{k+1} / \lambda_{k}$ is a partition of size $r$ where $\lambda_{k+1}$ and $\lambda_{k}$ have the same $r$-core. Any such partition must be an $r$-ribbon, and the result follows.

5.3. Existence and uniqueness. Suppose that $h(z)$ is a $C_{r}$-fixed polynomial of the form (1.2), where $\operatorname{val}\left(h_{1}\right)>\cdots>\operatorname{val}\left(h_{\ell}\right)$. We have shown that every point $x \in X_{\lambda / \mu}(h(z))$ has some associated tableau $T_{x}$ and that for a point in $X^{r}$ this tableau is a standard $r$-ribbon tableau.

Now, fix $T \in \operatorname{SRT}^{r}(\lambda / \mu)$, and let $\mu=\lambda_{0} \subsetneq \lambda_{1} \subsetneq \cdots \subsetneq \lambda_{\ell}=\lambda$ be the corresponding chain of partitions. To complete the proof of Theorem 1.7, we need to prove that there exists a unique point $x \in X_{\lambda / \mu}^{r}(h(z))$ such that $T_{x}=T$ and, moreover, that $x$ is a reduced point of $X_{\lambda / \mu}^{r}(h(z))$.

Suppose that such a point $x$ exists. Then the following must be true:

(1) $x \in X_{\lambda / \mu}^{r}$; equivalently by Theorem 4.3 and Theorem 4.8, $p_{\nu}(x)=0$ for $\nu \notin \Lambda_{\lambda / \mu}^{r, \kappa}$

(2) by Lemma $5.3(2)$

$$
\operatorname{LT}\left(p_{\lambda_{k}}(x)\right)=\frac{q_{\lambda}}{q_{\lambda_{k}}} \prod_{i=k+1}^{\ell} \operatorname{LT}\left(h_{i}\right) \quad \text { for } k=0, \ldots, \ell .
$$

We will first show that there exists a point in $X_{\lambda / \mu}^{r}$ satisfying these two conditions. Then we will show that the additional condition $\operatorname{Wr}(x ; z)=h(z)$ makes this point unique and reduced in $X_{\lambda / \mu}^{r}(h(z))$.

Let $\mathbf{w}$ be a weight vector constructed (as in Section 4.3) from the tableau $T$ and the decreasing sequence of rational numbers $\operatorname{val}\left(h_{1}\right), \ldots, \operatorname{val}\left(h_{\ell}\right)$. Note that $w_{\lambda_{k}}=\sum_{i=k+1}^{\ell} \operatorname{val}\left(h_{i}\right)$. Let

$$
Y:=\left\{x \in X_{\lambda / \mu}^{r} \mid \operatorname{val}\left(p_{\nu}(x)\right)=w_{\nu} \text { for all } \nu \in \Lambda_{\lambda, \mu}^{r, \kappa}\right\} .
$$

As we will see, the point $x \in X_{\lambda / \mu}^{r}(h(z))$ corresponding to $T$ will be in $Y$. Consider a vector $\boldsymbol{\alpha}=\left(\alpha_{\nu}\right)_{\nu \in \Lambda} \in \mathbb{F}^{\Lambda}$ satisfying the following conditions:

$$
\begin{aligned}
\alpha_{\nu} & =0 & & \text { if and only if } \nu \notin \Lambda_{\lambda / \mu}^{r, \kappa}, \\
\left(\epsilon_{\nu} \alpha_{\nu}\right)\left(\epsilon_{\sigma} \alpha_{\sigma}\right) & =\left(\epsilon_{\nu \vee \sigma} \alpha_{\nu \vee \sigma}\right)\left(\epsilon_{\nu \wedge \sigma} \alpha_{\nu \wedge \sigma}\right) & & \text { for } \nu, \sigma \in \Lambda_{\lambda / \mu}^{r, \kappa} .
\end{aligned}
$$

Here $\epsilon_{\nu}= \pm 1$ is the sign defined in Section 4.3. For any such $\boldsymbol{\alpha}$, let

$$
Y_{\boldsymbol{\alpha}}:=\left\{x \in Y \mid \operatorname{LT}\left(p_{\nu}(x)\right)=\alpha_{\nu} u^{w_{\nu}} \text { for all } \nu \in \lambda\right\} .
$$


Lemma 5.5. For any sequence $\beta_{0}, \ldots, \beta_{\ell} \in \mathbb{F}^{\times}$, there is a unique vector $\boldsymbol{\alpha} \in \mathbb{F}^{\Lambda}$ satisfying (5.13), (5.14) and $\alpha_{\lambda_{k}}=\beta_{k}$ for $k=0, \ldots, \ell$.

Proof. This follows from the fact that $\Lambda_{\lambda / \mu}^{r, \kappa}$ is a distributive lattice. Condition (5.13) asserts that $\nu \mapsto \epsilon_{\nu} \alpha_{\nu}$ is a modular function, and so this function is unique by its values on the maximal chain $\lambda_{0} \subsetneq \cdots \subsetneq \lambda_{\ell}$.

Lemma 5.6. If $x \in X_{\lambda / \mu}^{r}$ is a point such that

$$
L T\left(p_{\lambda_{k}}(x)\right)=\alpha_{\lambda_{k}} u^{w_{\lambda_{k}}} \quad \text { for } k=0, \ldots, \ell,
$$

then $x \in Y_{\boldsymbol{\alpha}}$.

Proof. Let $\alpha_{\nu}^{\prime}=\left[u^{w_{\nu}}\right] p_{\nu}(x)$. Since $p_{\nu}(x)=0$ for $\nu \notin \Lambda_{\lambda / \mu}^{r, \kappa}$, it is enough to show that $\operatorname{LT}\left(p_{\nu}(x)\right)=\alpha_{\nu} u^{w_{\nu}}$ for $\nu \in \Lambda_{\lambda / \mu}^{r, \kappa}$. Equivalently, we must show that $\operatorname{val}\left(p_{\lambda}(x)\right) \geq w_{\nu}$ and $\alpha_{\nu}^{\prime}=\alpha_{\nu} \neq 0$ for $\nu \in \Lambda_{\lambda / \mu}^{r, \kappa}$.

Note that $\alpha_{\nu}^{\prime}=0$ for $\nu \notin \Lambda_{\lambda / \mu}^{r, \kappa}$ and $\alpha_{\lambda_{k}}^{\prime}=\alpha_{\lambda_{k}}$ for $k=0, \ldots, \ell$. By Lemma 5.3(1), (5.8) holds, which can be re-expressed as

$$
\operatorname{val}\left(p_{\nu}(x)\right) \geq w_{\nu} \quad \text { for all } \nu \in \Lambda .
$$

Thus $\alpha_{\nu}^{\prime}=\left.p_{\nu}(x) u^{-w_{\nu}}\right|_{u=0}$. This implies that $\left(\alpha_{\nu}^{\prime}\right)_{\nu \in \Lambda}$ is in the variety of the initial ideal $\operatorname{In}_{\mathbf{w}}\left(I_{\lambda / \mu}^{r, \kappa}\right)$. By Lemma 4.15 we see that $\boldsymbol{\alpha}^{\prime}=\left(\alpha_{\nu}^{\prime}\right)_{\nu \in \Lambda}$ is a vector satisfying (5.13), (5.14) and $\alpha_{\lambda_{k}}^{\prime}=\alpha_{\lambda_{k}}$ for $k=0, \ldots, \ell$. By Lemma 5.5, we deduce that $\boldsymbol{\alpha}^{\prime}=\boldsymbol{\alpha}$, as required.

Lemma 5.7. There exist local coordinates $y_{1}, \ldots, y_{\ell}$ on an open subset of $X$ containing $Y$ with the following properties:

(1) For each $\nu \in \Lambda_{\lambda / \mu}^{r, \kappa}$, there exists a polynomial $P_{\nu}\left(y_{1}, \ldots, y_{\ell}\right) \in \mathbb{K}_{+}\left[y_{1}, \ldots, y_{\ell}\right]$ such that $p_{\nu}(x)=u^{w_{\nu}} P_{\nu}\left(y_{1}(x), \ldots, y_{\ell}(x)\right)$ for all $x \in Y$.

(2) $L T\left(p_{\lambda_{k}}(x)\right)=\epsilon_{\lambda_{k}} L T\left(y_{k+1}(x) \ldots y_{\ell}(x)\right) u^{w_{\lambda_{k}}}$ for all $x \in Y$.

(3) The map $x \mapsto\left(y_{1}(x), \ldots, y_{\ell}(x)\right)$ gives a bijection

$Y_{\boldsymbol{\alpha}} \leftrightarrow\left\{\left(y_{1}, \ldots, y_{\ell}\right) \in\left(\mathbb{K}_{+}\right)^{\ell} \mid L T\left(y_{k}\right)=\frac{\epsilon_{\lambda_{k}} \alpha_{\lambda_{k}}}{\epsilon_{\lambda_{k-1}} \alpha_{\lambda_{k-1}}}\right.$ for $\left.k=1, \ldots, \ell\right\}$.

In particular, $Y_{\boldsymbol{\alpha}}$ is non-empty, and $\operatorname{val}\left(y_{1}(x)\right)=\cdots=\operatorname{val}\left(y_{\ell}(x)\right)=0$ for all $x \in Y$.

Example 5.8. We illustrate the construction of the local coordinates $y_{1}, \ldots, y_{\ell}$ in the case where $r=1$. Suppose $d=3, n=7$, and

$$
T=\begin{array}{|l|l|l|l|}
\cline { 2 - 3 } & 2 & 4 & 7 \\
\hline 1 & 5 & 6 & 9 \\
\hline 3 & 8 & \multicolumn{3}{|c}{}
\end{array}
$$

For any point $x \in Y$, we can choose a basis for $x$ so that the matrix $A_{i j}$ of Section 4.1 has the form

$$
A=\left(\begin{array}{ccccccc}
0 & 0 & 0 & \hat{y}_{2} \hat{y}_{4} \hat{y}_{7} & \hat{y}_{4} \hat{y}_{7} & \hat{y}_{7} & 1 \\
0 & \hat{y}_{1} \hat{y}_{5} \hat{y}_{6} \hat{y}_{9} & \hat{y}_{5} \hat{y}_{6} \hat{y}_{9} & \hat{y}_{6} \hat{y}_{9} & \hat{y}_{9} & 1 & 0 \\
\hat{y}_{3} \hat{y}_{8} & \hat{y}_{8} & 1 & 0 & 0 & 0 & 0
\end{array}\right),
$$

in which the entries are 0 or products of indeterminates:

$$
A_{i j}=\prod_{m=j+i-d+1}^{\lambda^{i}+i-d+1} \hat{y}_{T_{i m}}
$$


where $T_{i m}$ denotes the entry of $T$ in row $i$, column $m$. The coordinates $y_{1}, \ldots, y_{\ell}$ are defined to be

$$
y_{k}(x):=u^{-\operatorname{val}\left(h_{k}\right)} \hat{y}_{k} \quad \text { for } k=1, \ldots, \ell .
$$

The reader can check that for any $h(z)$ with $\operatorname{val}\left(h_{1}\right)>\cdots>\operatorname{val}\left(h_{\ell}\right)$, properties (1)-(3) of Lemma 5.7 are satisfied.

Proof. When $r=1$, the construction of $y_{1}, \ldots, y_{\ell}$ is illustrated in Example 5.8. The proof that properties (1)-(3) hold for this construction is routine. When $r>1$, we use the identification

$$
X_{\lambda / \mu}^{r, \kappa} \cong \prod_{k=0}^{r-1} \widetilde{X}_{\widetilde{\lambda}_{k} / \widetilde{\mu}_{k}}
$$

of Lemma 4.12, and define $y_{1}, \ldots, y_{\ell}$ to be the local coordinates on appropriate subsets of $\widetilde{X}_{\widetilde{\lambda}_{0} / \widetilde{\mu}_{0}}, \ldots, \widetilde{X}_{\widetilde{\lambda}_{r-1} / \widetilde{\mu}_{r-1}}$. Specifically, if $i_{1}, \ldots, i_{m}$ are the entries of $T$ that correspond to boxes of $\square_{k}$ under the $r$-quotient construction, then $y_{i_{1}}, \ldots, y_{i_{m}}$ are the local coordinates (defined as in the $r=1$ case) on $\widetilde{X}_{\widetilde{\lambda}_{k} / \widetilde{\mu}_{k}}$. We leave it to the reader to verify that properties (1)-(3) can be deduced from the $r=1$ case.

We note that Lemmas 5.5, 5.6 and 5.7 (and their proofs) are still valid under the weaker condition $\operatorname{val}\left(h_{1}\right) \geq \cdots \geq \operatorname{val}\left(h_{\ell}\right)$, provided that Condition 4.13 is satisfied in the construction of the weight vector $\mathbf{w}$. We will need this fact in Section 6 .

Lemma 5.9. If $x \in Y$, then $T_{x}=T$.

Proof. For $x \in Y$ we have

$$
\operatorname{val}\left(p_{\lambda_{k}}(x)\right)=w_{\lambda_{k}}=\sum_{i=k+1}^{\ell} \operatorname{val}\left(h_{i}\right)<w_{\nu}=\operatorname{val}\left(p_{\nu}(x)\right)
$$

for every partition $\nu \in \Lambda_{\lambda / \mu}^{r, \kappa}$ such that $|\nu|=\left|\lambda_{k}\right|, \nu \neq \lambda_{k}$. The inequality here follows from the definition of $\mathbf{w}$. This shows that $T_{x}=T$.

Lemma 5.10. With $h(z)$ as above, let $X_{\lambda / \mu}$ be a compatible Richardson variety. For every $T \in \operatorname{SRT}^{r}(\lambda / \mu)$, there exists a unique point $x \in X_{\lambda / \mu}^{r}(h(z))$ whose corresponding tableau is $T_{x}=T$. Moreover, $X_{\lambda / \mu}^{r}(h(z))$ is reduced.

Proof. By Lemma 5.5 there is a unique $\boldsymbol{\alpha} \in \mathbb{F}^{\Lambda}$ satisfying (5.13), (5.14) and

$$
\alpha_{\lambda_{k}}=\frac{q_{\lambda}}{q_{\lambda_{k}}} \prod_{i=k+1}^{\ell} \operatorname{LC}\left(h_{i}\right) \quad \text { for } k=0, \ldots, \ell .
$$

For this $\boldsymbol{\alpha}$, (5.11) is equivalent to (5.16). Thus if $x \in X_{\lambda / \mu}^{r}(h(z))$ is a point such that $T_{x}=T$, then by Lemma 5.6 we must have $x \in Y_{\boldsymbol{\alpha}}$. Conversely, by Lemma 5.9 for every point $x \in Y_{\boldsymbol{\alpha}}$ we have $T_{x}=T$. It therefore suffices to show that there exists a unique, reduced point in $Y_{\boldsymbol{\alpha}} \cap X_{\lambda / \mu}^{r}(h(z))$.

Let

$$
H:=\left\{g(z) \in \mathbb{K}_{N}[z] \mid \operatorname{LT}\left(\left[z^{j}\right] g(z)\right)=\operatorname{LT}\left(\left[z^{j}\right] h(z)\right) \text { for } j=0, \ldots, N\right\} .
$$


We now show that $\operatorname{Wr}\left(Y_{\boldsymbol{\alpha}}\right) \subset H$. For any $x \in Y_{\boldsymbol{\alpha}}$ we have

$$
\begin{aligned}
\operatorname{LT}\left(\left[z^{j}\right] \operatorname{Wr}(x ; z)\right) & =\frac{1}{q_{\lambda}} \operatorname{LT}\left(\left[z^{j}\right] \sum_{\nu \vdash j} q_{\nu} p_{\nu}(x)\right) \\
& = \begin{cases}\frac{q_{\lambda_{k}}}{q_{\lambda}} \operatorname{LT}\left(p_{\lambda_{k}}(x)\right) & \text { if } j=\left|\lambda_{k}\right| \text { for some } k, \\
0 & \text { otherwise, }\end{cases}
\end{aligned}
$$

since, in the first case, $T_{x}=T$, so $p_{\lambda_{k}}(x)$ has unique minimal valuation among all Plücker coordinates indexed by partitions of size $j=\left|\lambda_{k}\right|$. On the other hand, if $j=\left|\lambda_{k}\right|$,

$$
\begin{aligned}
\operatorname{LT}\left(\left[z^{j}\right] h(z)\right) & =\prod_{i=k+1}^{\ell} \operatorname{LT}\left(h_{i}\right) \\
& =\frac{q_{\lambda_{k}}}{q_{\lambda}} \alpha_{\lambda_{k}} u^{w_{\lambda_{k}}} \\
& =\frac{q i \lambda_{k}}{q_{\lambda}} \operatorname{LT}\left(p_{\lambda_{k}}(x)\right),
\end{aligned}
$$

and $\left[z^{j}\right] h(z)=0$ otherwise. Thus $\operatorname{Wr}(x ; z) \in H$, for all $x \in Y_{\boldsymbol{\alpha}}$. To finish, we show that the map $\operatorname{Wr}: Y_{\boldsymbol{\alpha}} \rightarrow H$ is invertible. For this we write $\operatorname{Wr}(x ; z)=h(z)$ as a system of equations in the local coordinates $y_{1}, \ldots, y_{\ell}$ of Lemma 5.7, and use Hensel's Lemma (see e.g. [4, Exer. 7.25]), which is the analogue of the inverse function theorem for valuation rings.

Comparing non-zero coefficients of both sides, $\operatorname{Wr}(x ; z)=h(z)$ can be written as

$$
\sum_{\nu \vdash(|\mu|+r k)} q_{\nu} p_{\nu}(x)=\left[z^{|\mu|+r k}\right] h(z) \quad \text { for } k=0, \ldots, \ell .
$$

By Lemma 5.7(2), the leading term of the left hand side of (5.19) is

$$
q_{\lambda_{k}} \operatorname{LT}\left(p_{\lambda_{k}}(x)\right)=q_{\lambda_{k}} \epsilon_{\lambda_{k}} \operatorname{LT}\left(y_{k+1}(x) \cdots y_{\ell}(x)\right) u^{w_{\lambda_{k}}} .
$$

From (5.18), the leading term of the right hand side of (5.19) is

$$
q_{\lambda_{k}} \alpha_{\lambda_{k}} u^{w_{\lambda_{k}}} \text {. }
$$

Dividing both sides by $\epsilon_{\lambda_{k}} q_{\lambda_{k}} u^{w_{\lambda_{k}}}$, the system of equations (5.19) can be written as

$$
y_{k+1} \cdots y_{\ell}=\epsilon_{\lambda_{k}} \alpha_{\lambda_{k}}+u^{\delta_{k}} G_{k}\left(y_{1}, \ldots, y_{\ell}\right) \quad \text { for } k=0, \ldots, \ell,
$$

where $\delta_{k}>0$, and by Lemma $\left[5.7(1) G_{k}\left(y_{0}, \ldots, y_{\ell}\right) \in \mathbb{K}_{+}\left[y_{1}, \ldots, y_{\ell}\right]\right.$. To apply Hensel's Lemma to the system (5.22), we need to check two things.

(1) Every $x \in Y_{\boldsymbol{\alpha}}$ is a first order solution to (5.22); that is, $\operatorname{LT}\left(y_{k+1}(x) \cdots y_{\ell}(x)\right)$ $=\epsilon_{\lambda_{k}} \alpha_{\lambda_{k}}$ for $x \in Y_{\boldsymbol{\alpha}}$. This follows from Lemma [5.7(3).

(2) The Jacobian matrix $\left(\frac{\partial}{\partial y_{j}} y_{i+1} \cdots y_{\ell}\right)_{i, j=1, \ldots, \ell}$ is invertible over $\mathbb{K}_{+}$, for $x \in$ $Y_{\boldsymbol{\alpha}}$. This is true because the Jacobian matrix is upper-triangular, and the diagonal entries are invertible by Lemma $5.7(3)$.

We deduce that the system (5.22) has a unique, reduced solution $\left(y_{1}, \ldots, y_{\ell}\right) \in$ $\left(\mathbb{K}_{+}\right)^{\ell+1}$. This solution gives the local coordinates of the point $x \in Y_{\boldsymbol{\alpha}} \cap X_{\lambda / \mu}^{r}(h(z))$, as required.

Lemma $[5.10$ is the final ingredient in the proof of Theorem 1.7. 
Proof of Theorem 1.7. Since $h(z)$ is assumed to be a generic polynomial of the form (1.2), we may assume that $\operatorname{val}\left(h_{1}\right)>\cdots>\operatorname{val}\left(h_{\ell}\right)$. Lemmas 5.4 and 5.10 show that in this case, $x \mapsto T_{x}$ gives a bijection between the reduced finite scheme $X_{\lambda / \mu}^{r}(h(z))$ and $\operatorname{SRT}^{r}(\lambda / \mu)$.

Remark 5.11. Although $X_{\lambda / \mu}^{r}(h(z))$ is reduced, it is not always true that $X_{\lambda / \mu}(h(z))$ is reduced when $h(z)$ is a generic polynomial of the form (1.2). One can see this from the combinatorics. For example, suppose $r=3, h(z)=z^{3}+1$ and $\lambda / \mu=21$. Then $|\operatorname{SYT}(\lambda / \mu)|=2$, and $\left|\operatorname{SRT}^{3}(\lambda / \mu)\right|=1$, which means that $X_{\lambda / \mu}(h(z))$ is a finite scheme of length 2 , and $C_{3}$ acts on it with exactly 1 fixed point. Since $C_{3}$ cannot act on a set of size 2 with only one fixed point, this shows that $X_{\lambda / \mu}(h(z))$ is supported on a single point, hence is non-reduced. More generally, if $r$ is prime and $|\operatorname{SYT}(\lambda / \mu)| \not \equiv\left|\mathrm{SRT}^{r}(\lambda / \mu)\right|(\bmod r)$, then for every $C_{r}$-fixed $h(z)$ compatible with $X_{\lambda / \mu}$, we have that $X_{\lambda / \mu}(h(z))$ is non-reduced.

\section{Dihedral group aCtions}

6.1. $D_{r}$-fixed points. Our final goal in this paper is to prove Theorem 1.10, We begin with some simple observations about $D_{r}$-fixed points in $X$ and $D_{r}$-fixed polynomials in $\mathbb{P}\left(\mathbb{K}_{N}[z]\right)$.

For any point $x \in X$, let $x^{\vee}:=\left(\begin{array}{ll}0 & 1 \\ 1 & 0\end{array}\right) x$.

Proposition 6.1. The Plücker coordinates of $x^{\vee}$ are

$$
p_{\lambda}\left(x^{\vee}\right)=p_{\lambda \vee}(x), \quad \lambda \in \Lambda .
$$

We note once again that the Plücker coordinates are homogeneous coordinates, and hence (6.1) must be viewed as a system of equations that holds up to a scalar multiple.

Proof. The action of $\left(\begin{array}{ll}0 & 1 \\ 1 & 0\end{array}\right)$ on a polynomial $\mathbb{C}_{n-1}[z]$ is given by

$$
\left(\begin{array}{ll}
0 & 1 \\
1 & 0
\end{array}\right)\left(\alpha_{n-1} z^{n-1}+\alpha_{n-2} z^{n-2}+\cdots+\alpha_{0}\right)=\alpha_{0} z^{n-1}+\cdots+\alpha_{n-2} z+\alpha_{n-1} .
$$

In terms of the matrix $A_{i j}$ used to defined the Plücker coordinates (see Section 4.1), the action of $\left(\begin{array}{ll}0 & 1 \\ 1 & 0\end{array}\right)$ simply reverses the order of the columns; hence the minor $A_{J\left(\lambda^{\vee}\right)}$ for $x$ becomes $(-1)^{d(d-1) / 2} A_{J(\lambda)}$ for $x^{\vee}$. These are the Plücker coordinates $p_{\lambda \vee}(x)$ and $p_{\lambda}\left(x^{\vee}\right)$, respectively. The sign is a constant scalar multiple, so it can be discarded.

Proposition 6.2. If $x \in X$ is a $D^{r}$ fixed point, and $X_{\lambda / \mu}$ is the minimal Richardson variety containing $x$, then $\mu=\lambda^{\vee}$.

Proof. Since $x=x^{\vee}=\left(\begin{array}{ll}0 & 1 \\ 1 & 0\end{array}\right) x, p_{\nu^{\vee}}(x)=p_{\nu}(x)$ for all $\nu \in \Lambda$. In particular, if $\lambda$ is the maximal partition such that $p_{\lambda}(x) \neq 0$, then $\lambda^{\vee}$ is the minimal partition such that $p_{\lambda \vee}(x) \neq 0$. By Proposition 4.2, we must have $\mu=\lambda^{\vee}$.

This establishes one easy special case of Theorem 1.10. If $\mu \neq \lambda^{\vee}$, then there are no $D_{r}$-fixed points in $X_{\lambda / \mu}(h(z))$, and the combinatorial sets $\operatorname{RRT}^{r}(\lambda / \mu)$ and $\widehat{\operatorname{RRT}}^{r}(\lambda / \mu)$ are both empty.

Let $h(z) \in \mathbb{P}\left(\mathbb{K}_{N}[z]\right)$ be a $D_{r}$-fixed polynomial, and let $X_{\lambda / \mu}$ be a compatible Richardson variety. Henceforth, we will assume that $\mu=\lambda^{\vee}$. Working over the field $\mathbb{K}$, we will write $h(z)$ in the form (1.2), where $m=|\mu|, r \ell=|\lambda / \mu|$, and

$$
\operatorname{val}\left(h_{1}\right) \geq \operatorname{val}\left(h_{2}\right) \geq \cdots \geq \operatorname{val}\left(h_{\ell}\right) \text {. }
$$


TABLE 6.1. The three generic types of $D_{r}$-fixed polynomials over $\mathbb{K}$.

\begin{tabular}{|c|c|c|}
\hline Type (1) & Type $(2)$ & Type $(3)$ \\
\hline$\ell$ is odd & $\ell$ is even & $\ell$ is even \\
$\operatorname{val}\left(h_{1}\right)>\cdots>\operatorname{val}\left(h_{\ell}\right)$ & $\operatorname{val}\left(h_{1}\right)>\cdots>\operatorname{val}\left(h_{\ell}\right)$ & $\operatorname{val}\left(h_{1}\right)>\cdots>\operatorname{val}\left(h_{\ell / 2}\right)$ \\
$h_{(\ell+1) / 2}= \pm 1$ & $h_{\ell / 2}, h_{(\ell / 2)+1} \neq \pm 1$ & $h_{\ell / 2}=1$ and $h_{(\ell / 2)+1}=-1$ \\
$h_{\ell+1-k}=\left(h_{k}\right)^{-1}$ & $h_{\ell+1-k}=\left(h_{k}\right)^{-1}$ & $h_{\ell+1-k}=\left(h_{k}\right)^{-1}$ \\
for $k=1, \ldots, \frac{\ell-1}{2}$ & for $k=1, \ldots, \frac{\ell}{2}$ & for $k=1, \ldots, \frac{\ell}{2}-1$ \\
\hline
\end{tabular}

The space of all $h(z)$ that are compatible with $X_{\lambda / \mu}$ always has two components. If $\ell$ is odd, the analysis will be the same for both components, and we treat them as a single case. If $\ell$ is even, the analysis is different for the two components. Hence we will need to consider three different cases, corresponding to the three different generic types of $D_{r}$-fixed polynomials described in the introduction.

In type (1), $\ell$ is odd, and we can assume the $h_{1}, \ldots, h_{\ell}$ have distinct valuations. Since $\left\{h_{1}, \ldots, h_{\ell}\right\}$ is invariant under $z \mapsto \frac{1}{z}$, this implies that $h_{(\ell+1) / 2}= \pm 1$, and $h_{\ell+1-k}=\left(h_{k}\right)^{-1}$ for $k=1, \ldots, \frac{\ell-1}{2}$. Similarly in type $(2)$, where $\ell$ is even, we may assume that $h_{1}, \ldots, h_{\ell}$ have distinct valuations. Hence $h_{\ell+1-k}=\left(h_{k}\right)^{-1}$ for $k=1, \ldots, \frac{\ell}{2}$, and none are equal to \pm 1 . In type $(3), \ell$ is even, and we are required to have $h_{\ell / 2}=1$ and $h_{(\ell / 2)+1}=-1$, which both have valuation 0 . The rest of the $h_{i}$ can be assumed to have distinct valuations, and hence $h_{\ell+1-k}=\left(h_{k}\right)^{-1}$ for $k=1, \ldots, \frac{\ell}{2}-1$. A summary is given in Table 6.1

Proposition 6.3. The $D_{r}$-fixed polynomials of types (1), (2) or (3) form a Zariskidense subset of all $D_{r}$-fixed polynomials in $\mathbb{P}\left(\mathbb{K}_{N}[z]\right)$ that are compatible with $X_{\lambda / \mu}$.

Proof. Let $h(z) \in \mathbb{P}\left(\mathbb{K}_{N}[z]\right)$ be a polynomial compatible with $X_{\lambda / \mu}$. For $a \in \mathbb{K}$, let $M(a)$ denote the largest integer for which $\left(z^{r}+a\right)^{M(a)}$ divides $h(z)$. If $a \neq \pm 1$, then $M(a)=M\left(a^{-1}\right)$. It follows that $\ell-M(1)-M(-1)$ is even. There are four cases.

(1) If $\ell$ is odd, $M(1)$ is odd, and $M(-1)$ is even, then $h(z)$ is in the closure of the polynomials of type (1) for which $h_{(\ell+1) / 2}=1$.

(2) If $\ell$ is odd, $M(1)$ is even, and $M(-1)$ is odd, then $h(z)$ is in the closure of the polynomials of type $(1)$ for which $h_{(\ell+1) / 2}=-1$.

(3) If $\ell$ is even, $M(1)$ is even, and $M(-1)$ is even, then $h(z)$ is in the closure of the polynomials of type (2).

(4) If $\ell$ is even, $M(1)$ is odd, and $M(-1)$ is odd, then $h(z)$ is in the closure of the polynomials of type (3).

6.2. Reflection on the fibre models rotation of tableaux. We continue to assume that $h(z)$ is a $D_{r}$-fixed polynomial, and $X_{\lambda / \mu}$ is a compatible Richardson variety with $\mu=\lambda^{\vee}$. For any tableau $T$ of shape $\lambda / \mu$, let $T^{\vee}$ denote the tableau obtained by rotating $T$ by $180^{\circ}$, and replacing each entry $k$ by $m+1-k$, where $m$ is the largest entry.

Lemma 6.4. Let $x \in X(h(z))$ be any point, and $T_{x}$ be the associated tableau, as in Section [5.1, Then the tableau associated to $x^{\vee}$ is $\left(T_{x}\right)^{\vee}$. 
Thus the action of $\left(\begin{array}{ll}0 & 1 \\ 1 & 0\end{array}\right)$ on the fibre $X(h(z))$ models the action of a $180^{\circ}$ rotation on the tableaux. We note that this result does not require $h(z)$ to be generic, of one of the three types, though for our applications it will be.

Proof. Let $\lambda_{0} \subsetneq \lambda_{1} \subsetneq \cdots \subsetneq \lambda_{\ell}$ be the chain of partitions associated to $T_{x}$. Then $p_{\lambda_{k}}(x)$ has unique minimal valuation among all Plücker coordinates of $x$ indexed by partitions of size $\left|\lambda_{k}\right|$. By (6.1),$p_{\lambda_{k}^{\vee}}\left(x^{\vee}\right)$ has unique minimal valuation among all Plücker coordinates of $x^{\vee}$ indexed by partitions of size $\left|\lambda_{k}^{\vee}\right|$. Thus $\lambda_{\ell}^{\vee} \subsetneq \lambda_{\ell-1}^{\vee} \subsetneq$ $\cdots \subsetneq \lambda_{0}^{\vee}$ encodes $T_{x^{\vee}}$, i.e. $\left(T_{x}\right)^{\vee}=T_{x^{\vee}}$.

We immediately obtain the first case of Theorem 1.10.

Proof of Theorem 1.10(i). If $h(z)$ is of type (1) or (2), then $h_{1}, \ldots, h_{\ell}$ have distinct valuations, and hence by Lemma 5.10 we have a bijective correspondence between the reduced finite scheme $X_{\lambda / \mu}^{r}(h(z))$ and $\operatorname{SRT}^{r}(\lambda / \mu)$. For any $x \in X_{\lambda / \mu}^{r}(h(z))$, we have $x=x^{\vee}$ iff $T_{x}=T_{x^{\vee}}=\left(T_{x}\right)^{\vee}$, where the last equality is by Lemma 6.4. Hence $\left(\begin{array}{ll}0 & 1 \\ 1 & 0\end{array}\right)$-fixed points in $X_{\lambda / \mu}^{r}(h(z))$ are in bijection with the set $\operatorname{RRT}^{r}(\lambda / \mu)$ of rotationally-invariant $r$-ribbon tableaux.

If $h(z)$ is of type (3), then a bit more work is required, since $h_{\ell / 2}$ and $h_{(\ell / 2)+1}$ have the same valuation. In this case, the corresponding tableau is not a standard $r$-ribbon tableau, but something close. Let $T$ be a tableau of shape $\lambda / \mu$, where $|\lambda / \mu|=r \ell$ and $\ell$ is even. We say $T$ is an almost-standard $r$-ribbon tableau if the entries of $T$ not equal to $\frac{\ell}{2}$ form $r$-ribbons, and the entries equal to $\frac{\ell}{2}$ form a skew shape with $2 r$-boxes that supports an $r$-ribbon. We will write the chain of partitions corresponding to such a tableau $T$ as

$$
\mu=\lambda_{0} \subsetneq \cdots \subsetneq \lambda_{(\ell / 2)-1} \subsetneq \lambda_{(\ell / 2)+1} \subsetneq \cdots \subsetneq \lambda_{\ell}=\lambda .
$$

Lemma 6.5. Let $x \in X_{\lambda / \mu}^{r}(h(z))$, where $h(z)$ is of type (3). Then the corresponding $T_{x}$ is an almost-standard $r$-ribbon tableau.

Proof. The argument is essentially the same as the proof of Lemma 5.4.

One possibility, if $T$ is an almost-standard $r$-ribbon tableau of shape $\lambda / \mu$, is that the entries equal to $\frac{\ell}{2}$ actually form a $2 r$-ribbon. In this case we say that $T$ has a long ribbon.

Lemma 6.6. Let $T$ be an almost-standard r-ribbon tableau, and suppose $h(z)$ is of type (3)

(i) If T has a long ribbon, then there is exactly one point $x \in X_{\lambda / \mu}^{r}(h(z))$ such that $T_{x}=T$; moreover, $x$ is a reduced point of $X_{\lambda / \mu}^{r}(h(z))$.

(ii) If $T$ does not have a long ribbon, then there are at most two points in $X_{\lambda / \mu}^{r}(h(z))$ corresponding to $T$.

Proof. Consider a polynomial $h_{\mathbf{b}}(z)$ of the form

$$
h_{\mathbf{b}}(z)=z^{|\mu|} \prod_{i=1}^{\ell}\left(z^{r}+b_{i} h_{i}\right),
$$

where $\mathbf{b}=\left(b_{1}, \ldots, b_{\ell}\right) \in\left(\mathbb{F}^{\times}\right)^{\ell}$. We claim that if $b_{1}, \ldots, b_{\ell}$ are generic, then statements (i) and (ii) are true with $h(z)$ replaced by $h_{\mathbf{b}}(z)$. 
To prove the claim, we work over $\mathbb{F}=\mathbb{C}\{\{v\}\}$. Let

$$
\mathcal{U}=\left\{\left(v^{\gamma_{1}}, \ldots, v^{\gamma_{\ell}}\right) \mid \gamma_{k} \in \mathbb{Z}, \gamma_{1}>\cdots>\gamma_{\ell}\right\} .
$$

Fix $\mathbf{b}=\left(v^{\gamma_{1}}, \ldots, v^{\gamma_{\ell}}\right) \in \mathcal{U}$ and a small positive rational number $\varepsilon>0$. Put

$$
\hat{h}(z):=z^{|\mu|} \prod_{i=1}^{\ell}\left(z^{r}+v^{\gamma_{i}} u^{\varepsilon \gamma_{i}} h_{i}\right) .
$$

Arguing as in the proof of Lemma 5.3. we see that for any point $\hat{x} \in X_{\lambda / \mu}(\hat{h}(z))$ such that

$$
\operatorname{val}\left(p_{\lambda_{k}}(\hat{x})\right)=\sum_{i=k+1}^{\ell} \operatorname{val}\left(h_{i}\right)+O(\varepsilon) \quad \text { for } k=0, \ldots, \frac{\ell}{2}-1, \frac{\ell}{2}+1, \ldots, \ell,
$$

there is a corresponding point $x^{*}:=\Sigma(\hat{x})$ such that $T_{x^{*}}=T$. Conversely, if $\hat{x}$ does not satisfy (6.3), then $T_{x^{*}} \neq T$. Points $\hat{x} \in X_{\lambda / \mu}(\hat{h}(z))$ satisfying (6.3) are in bijection with tableaux $T \in \operatorname{SRT}^{r}(\lambda / \mu)$ whose associated chain of partitions is of the form

$$
\mu=\lambda_{0} \subsetneq \cdots \subsetneq \lambda_{(\ell / 2)-1} \subsetneq \lambda_{\ell / 2} \subsetneq \lambda_{(\ell / 2)+1} \subsetneq \cdots \subsetneq \lambda_{\ell}=\lambda
$$

for some $\lambda_{\ell / 2}$. In other words, these points correspond to extensions of the chain (6.2) to a maximal chain in $\Lambda_{\lambda / \mu}^{r, \kappa}$. If $T$ has a long ribbon, there is a unique way to extend this chain, hence one point in $X_{\lambda / \mu}^{r}\left(h_{\mathbf{b}}(z)\right)$ corresponding to $T$. If $T$ does not have a long ribbon, there are two ways to extend the chain, hence two corresponding points. This proves the claim.

Now, if $x^{*} \in X_{\lambda / \mu}^{r}\left(h_{\mathbf{b}}(z)\right)$ is a point such that $T_{x^{*}}=T$, then by Lemma 5.3(2), we have

$$
\operatorname{LT}\left(p_{\lambda_{k}}\left(x^{*}\right)\right)=\frac{q_{\lambda}}{q_{\lambda_{k}}} \prod_{i=k+1}^{\ell} \operatorname{LT}\left(b_{i} h_{i}\right) \quad \text { for } k=0, \ldots, \frac{\ell}{2}-1, \frac{\ell}{2}+1, \ldots, \ell .
$$

The right hand side of (6.4) is a nowhere-vanishing function of $b_{1}, \ldots, b_{\ell}$. This implies that property (6.4) is preserved when we take the limit as $\mathbf{b} \rightarrow(1, \ldots, 1)$. Thus in case (i) the limiting fibre $X_{\lambda / \mu}^{r}(h(z))$ contains exactly one reduced point $x$ such that $T_{x}=T$, which proves (i). In case (ii) the limiting fibre $X_{\lambda / \mu}^{r}(h(z))$ contains either:

(1) two distinct reduced points $x, x^{\prime}$ such that $T_{x}=T_{x^{\prime}}=T$ or

(2) a single point $x$ of multiplicity two such that $T_{x}=T$, which proves (ii).

Lemma 6.7. Let $T$ be an almost-standard $r$-ribbon tableau, and suppose $h(z)$ is of type (3). If $T$ does not have a long ribbon, then there are exactly two points $x, x^{\prime} \in$ $X_{\lambda / \mu}^{r}(h(z))$ such that $T_{x}=T_{x^{\prime}}=T$, and both are reduced points of $X_{\lambda / \mu}^{r}(h(z))$.

Proof. The proof uses essentially the same idea as the proof of Lemma 5.10, First, we identify a vector $\boldsymbol{\alpha} \in \mathbb{F}^{\Lambda}$ such that one of the points will lie in $Y_{\boldsymbol{\alpha}}$. Then we apply Hensel's Lemma to show that the point exists and is unique.

Let $\lambda_{\ell / 2}$ and $\lambda_{\ell / 2}^{\prime}$ denote the two partitions of size $\frac{N}{2}$ that extend the chain (6.2) to a maximal chain. Let $T^{+} \in \mathrm{SRT}^{r}(\lambda / \mu)$ be the tableau corresponding to the extended chain of partitions $\lambda_{0} \subsetneq \cdots \subsetneq \lambda_{\ell / 2} \subsetneq \cdots \subsetneq \lambda_{\ell}$, and let $\mathbf{w}$ be a weight vector obtained from $T^{+}$and the weakly decreasing sequence $\operatorname{val}\left(h_{1}\right), \ldots, \operatorname{val}\left(h_{\ell}\right)$. 
Then with $Y$ defined as in (5.12), we have $T_{x}=T$ for every $x \in Y$. The proof is analogous to that of Lemma 5.9

By Lemma 5.5 there is a unique $\boldsymbol{\alpha} \in \mathbb{F}^{\Lambda}$ satisfying (5.13), (15.14) and

$$
\begin{aligned}
\alpha_{\lambda_{k}} & =\frac{q_{\lambda}}{q_{\lambda_{k}}} \prod_{i=k+1}^{\ell} \mathrm{LC}\left(h_{i}\right) \quad \text { for } k=0, \ldots, \frac{\ell}{2}-1, \frac{\ell}{2}+1, \ldots, \ell, \\
\alpha_{\lambda_{\ell / 2}} & =\epsilon_{\circ} q_{\lambda}\left(\frac{q_{\lambda_{\ell / 2}^{\prime}}}{q_{\lambda_{(\ell / 2)-1}} q_{\lambda_{\ell / 2}} q_{\lambda_{(\ell / 2)+1}}}\right)^{\frac{1}{2}} \prod_{i=\frac{\ell}{2}+2}^{\ell} \operatorname{LC}\left(h_{i}\right),
\end{aligned}
$$

where $\epsilon_{\circ}=\left(\epsilon_{\lambda_{(\ell / 2)-1}} \epsilon_{\lambda_{\ell / 2}} \epsilon_{\lambda_{(\ell / 2)+1}} \epsilon_{\lambda_{\ell / 2}^{\prime}}\right)^{1 / 2}$. We may choose either square root.

Note that

$$
\lambda_{\ell / 2} \wedge \lambda_{\ell / 2}^{\prime}=\lambda_{(\ell / 2)-1} \quad \text { and } \quad \lambda_{\ell / 2} \vee \lambda_{\ell / 2}^{\prime}=\lambda_{(\ell / 2)+1},
$$

so from (5.14) we have

$$
\begin{aligned}
\alpha_{\lambda_{\ell / 2}^{\prime}} & =\left(\epsilon_{\circ}\right)^{2} \frac{\alpha_{\lambda_{(\ell / 2)-1}} \alpha_{\lambda_{(\ell / 2)+1}}}{\alpha_{\lambda_{\ell / 2}}} \\
& =\epsilon_{\circ} q_{\lambda}\left(\frac{q_{\lambda_{\ell / 2}}}{q_{\lambda_{(\ell / 2)-1}} q_{\lambda_{\ell / 2}^{\prime}} q_{\lambda_{(\ell / 2)+1}}}\right)^{\frac{1}{2}} \prod_{i=\frac{\ell}{2}}^{\ell} \mathrm{LC}\left(h_{i}\right)=-\frac{q_{\lambda_{\ell / 2}}}{q_{\lambda_{\ell / 2}^{\prime}}} \alpha_{\lambda_{\ell / 2}},
\end{aligned}
$$

where the last step uses $h_{\ell / 2}=1, h_{(\ell / 2)+1}=-1$.

For $j=0, \ldots, N$ and $g \in \mathbb{K}_{N}[z]$, let

$$
L_{j}(g(z)):= \begin{cases}\operatorname{LT}\left(\left[z^{j}\right] g(z)\right) & \text { if } j=\left|\lambda_{k}\right|, \operatorname{val}\left(\left[z^{j}\right] g(z)\right) \leq \sum_{i=k+1}^{\ell} \operatorname{val}\left(h_{i}\right), \\ 0 & \text { otherwise. }\end{cases}
$$

Note that $\operatorname{val}\left(\left[z^{\left|\lambda_{k}\right|}\right] h(z)\right) \geq \sum_{i=k+1}^{\ell} \operatorname{val}\left(h_{i}\right)$, with equality when $k \neq \frac{\ell}{2}$. On the other hand, there are two terms in the expansion of $\left[z^{N / 2}\right] h(z)$ that have this minimal valuation:

$$
\begin{aligned}
& {\left[u^{\sum_{i=(\ell / 2)+1}^{\ell} \operatorname{val}\left(h_{i}\right)} z^{N / 2}\right] h(z) } \\
= & {\left[u^{\sum_{i=(\ell / 2)+1}^{\ell} \operatorname{val}\left(h_{i}\right)}\right]\left(\left(h_{\ell / 2} h_{(\ell / 2)+2} \cdots h_{\ell}\right)+\left(h_{(\ell / 2)+1} h_{(\ell / 2)+2} \cdots h_{\ell}\right)\right)=0, }
\end{aligned}
$$

and so we have

$$
L_{j}(h(z))= \begin{cases}\operatorname{LT}\left(\left[z^{j}\right] h(z)\right) & \text { if } j \neq \frac{N}{2} \\ 0 & \text { if } j=\frac{N}{2}\end{cases}
$$

Define

$$
H:=\left\{g(z) \in \mathbb{K}_{N}[z] \mid L_{j}(g(z))=L_{j}(h(z)) \text { for } j=0, \ldots, N\right\} .
$$

We now show that $\operatorname{Wr}\left(Y_{\boldsymbol{\alpha}}\right) \subset H$. Let $x \in Y_{\boldsymbol{\alpha}}$. For $j \neq N / 2$, we have

$$
L_{j}(\operatorname{Wr}(x ; z))=\operatorname{LT}\left(\left[z^{j}\right] \operatorname{Wr}(x ; z)\right)=\operatorname{LT}\left(\left[z^{j}\right] h(z)\right)=L_{j}(h(z)) ;
$$

this is the same calculation as in the proof of Lemma 5.10. For $j=N / 2$, we have

$$
\operatorname{val}\left(p_{\lambda_{\ell / 2}}(x)\right)=\operatorname{val}\left(p_{\lambda_{\ell / 2}^{\prime}}(x)\right)=w_{\lambda_{\ell / 2}}=w_{\lambda_{\ell / 2}^{\prime}}=\sum_{i=\frac{\ell}{2}+1}^{\ell} \operatorname{val}\left(h_{i}\right)
$$


and all other Plücker coordinates of $x$ indexed by partitions of size $N / 2$ have strictly larger valuation. Thus we have

$$
\begin{aligned}
L_{N / 2}(\mathrm{Wr}(x ; z)) & =\sum_{\nu \vdash \frac{N}{2}} q_{\nu}\left[u^{\sum_{i=(\ell / 2)+1}^{\ell} \operatorname{val}\left(h_{i}\right)}\right] p_{\nu}(x) \\
& =q_{\lambda_{\ell / 2}}\left[u^{w_{\lambda_{\ell / 2}}}\right] p_{\lambda_{\ell / 2}}(x)+q_{\lambda_{\ell / 2}^{\prime}}\left[u^{\left.w_{\lambda_{\ell / 2}^{\prime}}\right]} p_{\lambda_{\ell / 2}^{\prime}}(x)\right. \\
& =q_{\lambda_{\ell / 2}} \alpha_{\lambda_{\ell / 2}}(x)+q_{\lambda_{\ell / 2}^{\prime}} \alpha_{\lambda_{\ell / 2}^{\prime}}(x) \\
& =0
\end{aligned}
$$

and hence $\operatorname{Wr}(x ; z) \in H$.

To complete the proof, we use Hensel's Lemma to show that Wr: $Y_{\boldsymbol{\alpha}} \rightarrow H$ is invertible. First, we write the system of equations (5.19) in terms of the local coordinates $y_{1}, \ldots, y_{\ell}$ of Lemma 5.7.

For $k \neq \frac{\ell}{2}$, leading term of the left hand side and right hand side of (5.19) are given by (5.20) and (5.21), respectively. When $k=\frac{\ell}{2}$, the leading term of the left hand side is

$$
\begin{aligned}
& q_{\lambda_{\ell / 2} \mathrm{LT}}\left(p_{\lambda_{\ell / 2}}(x)\right)+q_{\lambda_{\ell / 2}^{\prime}} \operatorname{LT}\left(p_{\lambda_{\ell / 2}^{\prime}}(x)\right) \\
& =q_{\lambda_{\ell / 2}} \epsilon_{\lambda_{\ell / 2}} \operatorname{LT}\left(y_{(\ell / 2)+1}(x) y_{(\ell / 2)+2}(x) \cdots y_{\ell}(x)\right) u^{w_{\lambda_{\ell / 2}}} \\
& \quad+q_{\lambda_{\ell / 2}^{\prime}} \epsilon_{\lambda_{\ell / 2}^{\prime}} \operatorname{LT}\left(y_{(\ell / 2)}(x) y_{(\ell / 2)+2}(x) \cdots y_{\ell}(x)\right) u^{w_{\lambda_{\ell / 2}^{\prime}}} .
\end{aligned}
$$

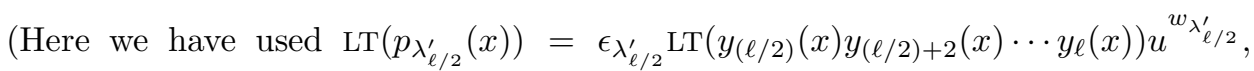
which comes from applying Lemma 5.7 with the chain of partitions

$$
\mu=\lambda_{0} \subsetneq \cdots \subsetneq \lambda_{(\ell / 2)-1} \subsetneq \lambda_{\ell / 2}^{\prime} \subsetneq \lambda_{(\ell / 2)+1} \subsetneq \cdots \subsetneq \lambda_{\ell}=\lambda .
$$

The local coordinates for this chain are the same as those for the chain $\lambda_{0} \subsetneq \cdots \subsetneq$ $\lambda_{\ell / 2} \subsetneq \cdots \subsetneq \lambda_{\ell}$, but the roles of $y_{\ell}$ and $y_{\ell / 2}$ are reversed.) By (6.8), the right hand side of (5.19) has valuation strictly greater than $w_{\lambda_{\ell / 2}}$ when $k=\frac{\ell}{2}$. Thus we can write the system (5.19) as

$$
\begin{gathered}
y_{k+1} \cdots y_{\ell}=\epsilon_{\lambda_{k}} \alpha_{\lambda_{k}}+u^{\delta_{k}} G_{k}\left(y_{1}, \ldots, y_{\ell}\right) \quad \text { for } k \neq \frac{\ell}{2}, \\
\left(\epsilon_{\lambda_{\ell / 2}^{\prime}} q_{\lambda_{\ell / 2}^{\prime}} y_{\ell / 2}+\epsilon_{\lambda_{\ell / 2}} q_{\lambda_{\ell / 2}} y_{(\ell / 2)+1}\right) y_{(\ell / 2)+2} \cdots y_{\ell}=u^{\delta_{\ell / 2}} G_{\ell / 2}\left(y_{1}, \ldots, y_{\ell}\right),
\end{gathered}
$$

where $\delta_{k}>0$, and $G_{k}\left(y_{0}, \ldots, y_{\ell}\right) \in \mathbb{K}_{+}\left[y_{1}, \ldots, y_{\ell}\right]$, for $k=0, \ldots, \ell$. Let

$$
F_{k}\left(y_{1}, \ldots, y_{\ell}\right):= \begin{cases}y_{k+1} \cdots y_{\ell} & \text { if } k \neq \frac{\ell}{2} \\ \left(\epsilon_{\lambda_{\ell / 2}^{\prime}} q_{\lambda_{\ell / 2}^{\prime}} y_{\ell / 2}+\epsilon_{\lambda_{\ell / 2}} q_{\lambda_{\ell / 2}} y_{(\ell / 2)+1}\right) y_{(\ell / 2)+2} \cdots y_{\ell} & \text { if } k=\frac{\ell}{2}\end{cases}
$$

We need to check two things.

(1) Every $x \in Y_{\boldsymbol{\alpha}}$ is a first order solution to (5.22). That is, for $x \in Y_{\boldsymbol{\alpha}}$,

$$
\left[u^{0}\right] F_{k}\left(y_{1}(x), \ldots, y_{\ell}(x)\right)= \begin{cases}\alpha_{\lambda_{k}} & \text { if } k \neq \frac{\ell}{2} \\ 0 & \text { if } k=\frac{\ell}{2}\end{cases}
$$


For $k \neq \frac{\ell}{2}$, this follows from Lemma $5.7(3)$. For $k=\frac{\ell}{2}$, we calculate

$$
\begin{aligned}
\operatorname{LT}\left(F _ { k } \left(y_{1}(x),\right.\right. & \left.\left.\ldots, y_{\ell}(x)\right)\right) & & \\
& =\operatorname{LT}\left(q_{\lambda_{\ell / 2}} p_{\lambda_{\ell / 2}}(x) u^{-w_{\lambda_{\ell / 2}}}+q_{\lambda_{\ell / 2}^{\prime}} p_{\lambda_{\ell / 2}^{\prime}}(x) u^{-w_{\lambda_{\ell / 2}}}\right) & & (\text { by (6.9) }) \\
& =\operatorname{LT}\left(q_{\lambda_{\ell / 2}} \alpha_{\lambda_{\ell / 2}}(x)+q_{\lambda_{\ell / 2}^{\prime}} \alpha_{\lambda_{\ell / 2}^{\prime}}(x)\right) & & (\text { by (5.15) }) \\
& =0 & & \text { (by (6.7)) }
\end{aligned}
$$

(2) The Jacobian matrix $\left(\frac{\partial}{\partial y_{j}} F_{i}\left(y_{1}, \ldots, y_{\ell}\right)\right)_{i, j=1, \ldots, \ell}$ is invertible over $\mathbb{K}_{+}$, for $x \in Y_{\boldsymbol{\alpha}}$. This is a block upper triangular matrix in which most of the diagonal blocks are $1 \times 1$ blocks and are invertible by Lemma 5.7)(3). However, there is one $2 \times 2$ diagonal block (in rows $\frac{\ell}{2}, \frac{\ell}{2}+1$ ) which is

$$
\left(\begin{array}{cc}
\epsilon_{\lambda_{\ell / 2}^{\prime}} q_{\lambda_{\ell / 2}^{\prime}} y_{(\ell / 2)+2} \cdots y_{\ell} & \epsilon_{\lambda_{\ell / 2}} q_{\lambda_{\ell / 2}} y_{(\ell / 2)+2} \cdots y_{\ell} \\
y_{(\ell / 2)+1} y_{(\ell / 2)+2} \cdots y_{\ell} & y_{\ell / 2} y_{(\ell / 2)+2} \cdots y_{\ell}
\end{array}\right) .
$$

This $2 \times 2$ matrix is invertible over $\mathbb{K}_{+}$if and only if

$$
\epsilon_{\lambda_{\ell / 2}^{\prime}} q_{\lambda_{\ell / 2}^{\prime}} \mathrm{LC}\left(y_{\ell / 2}\right) \neq \epsilon_{\lambda_{\ell / 2}} q_{\lambda_{\ell / 2}} \mathrm{LC}\left(y_{(\ell / 2)+1)}\right) \text {. }
$$

But by Lemma $5.7(3)$, this is equivalent to

$$
q_{\lambda_{\ell / 2}^{\prime}} \alpha_{\lambda_{\ell / 2}^{\prime}} \neq q_{\lambda_{\ell / 2}} \alpha_{\lambda_{\ell / 2}}
$$

which follows from (6.7), as $\alpha_{\lambda_{\ell / 2}} \neq 0$. Thus all diagonal blocks of the Jacobian matrix are invertible over $\mathbb{K}_{+}$, as required.

Therefore, by Hensel's Lemma the system (6.10) has a unique, reduced solution $\left(y_{1}, \ldots, y_{\ell}\right) \in\left(\mathbb{K}_{+}\right)^{\ell+1}$. This solution gives the local coordinates of a point $x \in$ $Y_{\boldsymbol{\alpha}} \cap X_{\lambda / \mu}^{r}(h(z))$; since $x \in Y$, we have $T_{x}=T$. We obtain the second point, $x^{\prime} \in X_{\lambda / \mu}^{r}(h(z))$, by replacing $\epsilon_{\circ}$ by $-\epsilon_{\circ}$ in the definition of (6.6) for $\alpha_{\lambda_{\ell / 2}}$. This shows that there are at least two reduced points in $X_{\lambda / \mu}^{r}(h(z))$ corresponding to $T$. By Lemma 6.6(ii), these are the only such points.

Lemma 6.8. Let $h(z)$ be a polynomial of type (3), and let $x \in X_{\lambda / \mu}^{r}(h(z))$. If $x=x^{\vee}$, then $T_{x}$ has a long ribbon.

Proof. Suppose to the contrary that $x=x^{\vee}$ but the tableau $T=T_{x}$ does not have a long ribbon. Let $\lambda_{0}, \ldots, \lambda_{\ell}$ and $\boldsymbol{\alpha}$ be as in the proof of Lemma 6.7 By Lemma 6.4 we must have $T=T^{\vee}$, so $\left(\lambda_{k}\right)^{\vee}=\lambda_{\ell-k}$, for $k \neq \frac{\ell}{2}$. It is not hard to see that we must also have $\left(\lambda_{\ell / 2}\right)^{\vee}=\lambda_{\ell / 2}$. By (6.1), $x=x^{\vee}$ implies that

$$
\frac{p_{\lambda_{k}}(x)}{p_{\left(\lambda_{k}\right)^{\vee}}(x)}
$$

is constant for all $k$. In particular, we must have

$$
\frac{p_{\lambda_{(\ell / 2)-1}}(x)}{p_{\left(\lambda_{(\ell / 2)+1}\right.}(x)}=\frac{p_{\lambda_{\ell / 2}}(x)}{p_{\lambda_{\ell / 2}}(x)}=1 .
$$

But from (5.15) and (6.5), we have

$$
\operatorname{LC}\left(\frac{p_{\lambda_{(\ell / 2)-1}}(x)}{p_{\left(\lambda_{(\ell / 2)+1}\right.}(x)}\right)=\frac{\alpha_{\lambda_{(\ell / 2)-1}}}{\alpha_{\lambda_{(\ell / 2)+1}}}=\frac{q_{\lambda_{(\ell / 2)+1}} \operatorname{LC}\left(h_{\ell / 2}\right) \operatorname{LC}\left(h_{(\ell / 2)+1}\right)}{q_{\lambda_{(\ell / 2)-1}}}<0,
$$

which gives a contradiction. 
We now obtain the second case of Theorem 1.10

Proof of Theorem 1.10(ii). If $h(z)$ is of type (3), then Lemmas 6.6 and 6.7 show that $X_{\lambda / \mu}^{r}(h(z))$ is reduced. Suppose $x=x^{\vee}$. Then $T_{x}$ must be an almoststandard ribbon tableau (by Lemma 6.5) with a long ribbon (by Lemma 6.8), and be rotationally-invariant (by Lemma 6.4). In other words, $T_{x} \in \widehat{\operatorname{RRT}}^{r}(\lambda / \mu)$. Conversely, if $T_{x} \in \widehat{\operatorname{RRT}}^{r}(\lambda / \mu)$, then $T_{x}=\left(T_{x}\right)^{\vee}=T_{x} \vee$. But by the uniqueness in Lemma 6.6(i) we have $x=x^{\vee}$. Thus $\left(\begin{array}{ll}0 & 1 \\ 1 & 0\end{array}\right)$-fixed points in $X_{\lambda / \mu}^{r}(h(z))$ are in bijection with $\widehat{\operatorname{RRT}}^{r}(\lambda / \mu)$.

\section{ACKNOWLEDGEMEnTS}

This work began with discussions at the October 2010 AIM workshop on algebraic systems with only real solutions. The author is grateful to all the organizers, staff and participants who made the workshop possible. He particularly wishes to thank Alexandre Eremenko, Frank Sottile and Alex Yong for the conversations that got everything started, and Brendon Rhoades for valuable correspondence and corrections.

\section{REFERENCES}

1. G. Benkart, F. Sottile and J. Stroomer, Tableau Switching: Algorithms and Applications, J. Combin. Theory Ser. A, 76 (1996), no. 1, 11-43. MR1405988 (97m:05261)

2. A. Eremenko and A. Gabrielov, Degrees of real Wronski maps, Disc. Comp. Geom., 28 (2002), 331-347. MR1923956 (2003g:14074)

3. A. Eremenko and A. Gabrielov, Counterexamples to pole placement by static output feedback, Linear Algebra Appl., 351/352 (2002), 211-218. MR1917479 (2003f:93045)

4. D. Eisenbud, Commutative algebra with a view toward algebraic geometry, Grad. Texts in Math., 150, 1995. MR.1322960 (97a:13001)

5. D. Eisenbud and J. Harris, Divisors on general curves and cuspidal rational curves, Invent. Math., 74 (1983), 371-418. MR724011 (85h:14019)

6. S. Fomin and N. Lulov, On the Number of Rim Hook Tableaux, Zapiski Nauchn. Sem. POMI, 223 (1995), 219-226. MR1374321 (97g:05168)

7. S. Fomin and D. Stanton, Rim hook lattices, Report No. 23, (1991/92) Inst. Mittag-Leffler.

8. W. Fulton, Young tableaux with applications to representation theory and geometry, Cambridge Univ. Press, New York, 1997. MR1464693 (99f:05119)

9. M. Haiman, Dual equivalence with applications, including a conjecture of Proctor, Discrete Math. 99 (1992), 79-113. MR1158783 (93h:05173)

10. J. Hauenstein, N. Hein, A. Martín del Campo, and F. Sottile, Beyond the Shapiro Conjecture and Eremenko-Gabrielov lower bounds, website (2010) www.math.tamu.edu/ $\sim$ sottile/research/pages/lower_Shapiro/index.html.

11. W.V.D. Hodge and D. Pedoe, Methods of algebraic geometry, Vol. II, Cambridge Univ. Press, 1952. MR 1288306 (95d:14002b)

12. G. James and A. Kerber, The representation theory of the symmetric group, Encyclopedia of Mathematics, 16, Addison-Wesley, 1981 MR644144(83k:20003)

13. V. Kharlamov and F. Sottile, Maximally inflected real rational curves, Mosc. Math. J., 3 (2003), no. 3, 947-987, 1199-1200. MR2078569 (2005f:14109)

14. A. Knutson, T. Lam and D. Speyer, Projections of Richardson Varieties, preprint, arXiv: 1008.3939.

15. A. Lascoux, B. Leclerc and J.-Y. Thibon, Ribbon tableaux, Hall-Littlewood functions, quantum affine algebras and unipotent varieties, J. Math. Phys., 38 (1997), 1041-1068. MR.1434225 (98c:05167)

16. M. van Leeuwen, Some bijective correspondences involving domino tableaux, Elec. J. Combin., 7 (2000), no. 1, R35 (electronic). MR1769066 (2001d:05195)

17. E. Miller and B. Sturmfels, Combinatorial commutative algebra, Grad. Texts in Math., 227, 2005. MR2110098 (2006d:13001) 
18. E. Mukhin, V. Tarasov and A. Varchenko, The B. and M. Shapiro conjecture in real algebraic geometry and the Bethe Ansatz, Ann. Math. 170 (2009), no. 2, 863-881. MR2552110 (2011b:17065)

19. E. Mukhin, V. Tarasov and A. Varchenko, Schubert calculus and representations of general linear group, J. Amer. Math. Soc. 22 (2009), no. 4, 909-940. MR2525775 (2011a:14099)

20. K. Purbhoo, Jeu de taquin and a monodromy problem for Wronksians of polynomials, Adv. Math. 224 (2010), no. 3, 827-862. MR2628796 (2011e:14101)

21. K. Purbhoo, Reality and transversality for Schubert calculus in OG( $n, 2 n+1)$, Math. Res. Lett. 17 (2010), no. 6, 1041-1046. MR2729628

22. K. Purbhoo, The Wronski map and shifted tableau theory, Int. Math. Res. Not. (to appear).

23. B. Rhoades, Cyclic sieving, promotion, and representation theory, J. Combin. Theory Ser. A, 117 (2010), no. 1, 38-76. MR2557880 (2011c:05369)

24. F. Sottile, Frontiers of reality in Schubert calculus, Bull. Amer. Math. Soc. 47 (2010), no. 1, 31-71. MR2566445 (2011f:14089)

25. B. Sagan, The symmetric group: Representations, combinatorial algorithms and symmetric functions, Grad. Texts in Math. 203, 2001. MR1824028 (2001m:05261)

26. B. Sagan, The cyclic sieving phenomenon: A survey, London Math. Soc. Lecture Note Ser. (to appear).

27. D. Stanton and D. White, A Schensted algorithm for rim-hook tableaux, J. Combin. Theory Ser. A, 40 (1985), 211-247. MR814412 (87c:05014)

28. R. Stanley, Enumerative combinatorics, vol. 2, Cambridge University Press, Cambridge, 1999. MR.1676282(2000k:05026)

29. J. Stembridge, Canonical bases and self-evacuating tableaux, Duke Math. J., 82 (1996), no. 3, 585-606. MR 1387685 (97f:05193)

30. B. Westbury, Invariant tensors and the cyclic sieving phenomenon, preprint, arXiv:0912.1512.

Department of Combinatorics and Optimization, University of Waterloo, Waterloo, ONTARIO, CANADA

E-mail address: kpurbhoo@math.uwaterloo.ca 